\title{
WHEN DO PEOPLE LIE, FOR WHOM, AND WHY? ALTRUISTIC LYING IN AN
}

\section{ALIBI CORROBORATION CONTEXT}

\author{
by \\ Stéphanie B. Marion, \\ Master of Arts, Ryerson University, 2010 \\ Bachelor of Science, University of Toronto, 2008
}

\author{
A Dissertation \\ presented to Ryerson University \\ in partial fulfillment of the \\ requirements for the degree of \\ Doctor of Philosophy \\ in the Program of \\ Psychology
}

Toronto, Ontario, Canada, 2014

(C) Stephanie B. Marion, 2014 


\section{AUTHOR'S DECLARATION FOR ELECTRONIC SUBMISSION OF A DISSERTATION}

I hereby declare that I am the sole author of this dissertation. This is a true copy of the dissertation, including any required final revisions, as accepted by my examiners.

I authorize Ryerson University to lend this dissertation to other institutions or individuals for the purpose of scholarly research

I further authorize Ryerson University to reproduce this dissertation by photocopying or by other means, in total or in part, at the request of other institutions or individuals for the purpose of scholarly research.

I understand that my dissertation may be made electronically available to the public. 


\title{
WHEN DO PEOPLE LIE, FOR WHOM, AND WHY? ALTRUISTIC LYING IN AN ALIBI CORROBORATION CONTEXT
}

\author{
Doctor of Philosophy, 2014 \\ Stéphanie B. Marion \\ Psychology \\ Ryerson University
}

\begin{abstract}
Three studies were conducted in order to identity factors that impact the likelihood that a witness will lie for a suspect in an alibi corroboration context. Specifically, the level of affinity between a suspect and a witness, the level of social pressure, and gender were investigated as factors impacting the likelihood that a witness would knowingly support a false alibi. During a study session purportedly intended to investigate dyadic problemsolving ability, a mock theft was staged in an adjacent office. When questioned by the experimenter, undergraduate students were provided the opportunity to either corroborate or refute a confederate's false alibi that the latter had been in the testing room during the time of the theft, which participants knew was false. In study 1, participants who were explicitly asked to conceal the confederate's whereabouts during the time of the theft were more likely to lie for him or her by corroborating the false alibi (61\% vs. $26 \%$ of those who were not asked to lie). In study 2 , there was a higher percentage of male participants who corroborated a male confederate's false alibi (41\%) compared to female participants who corroborated a female confederate's false alibi (23\%). In study 3 , participants were found to be more likely to lie for a confederate when the latter was their friend $(41 \%)$ than when he or she was a stranger $(18 \%)$. How much a participant liked the suspect (study 1) and whether or not the suspect had previously helped the participant (study 2) did not affect the rates of false alibi corroboration. The results confirm that alibi witnesses often lie, but suggest that investigators and jurors may underestimate the frequency with which strangers and acquaintances lie for one another, and that witnesses who lie do so more often because they trust that the suspect is innocent rather than guilty.
\end{abstract}




\section{ACKNOWLEDGEMENTS}

First and foremost, tremendous thanks to my supervisor Tara Burke for her excellent advice on this work, and for her continuous support and encouragement over the past six years. I also thank my advisory committee members, Stephen Want and Alasdair Goodwill, for their time and valuable recommendations.

Also a big thanks to my numerous research assistants for their time, commitment, and acting skills, which were invaluable for the collection of this data: Jessica, Patryce, Christine, Tyler, Justin, Ryan, Nydia, Andrew, Sepideh, and Alexei.

Finally, thanks go out to my lab mates Sami, Sara, and Stu, for their patience and understanding when I took over most of the lab space during my data collection. 


\section{Dedication}

To my parents and husband. Thank you for all of your love, support, and encouragement throughout my graduate training. 
Table of Contents

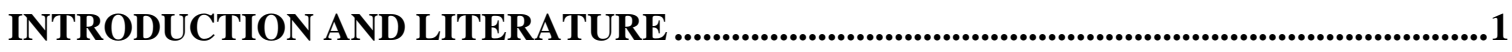

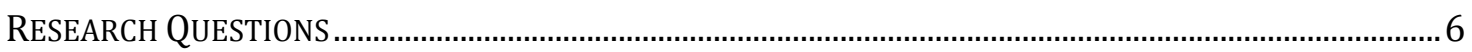

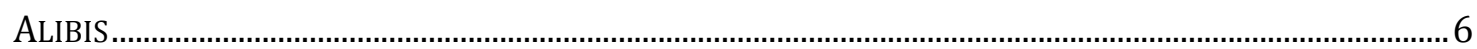

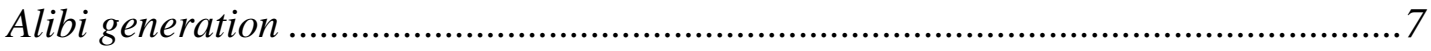

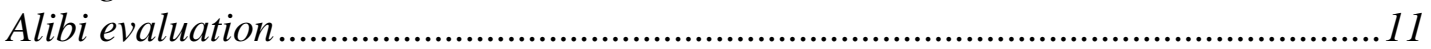

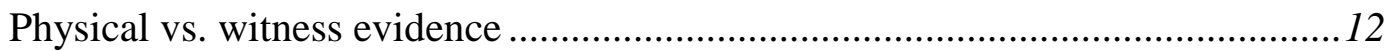

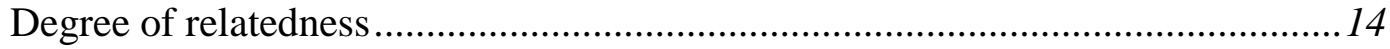

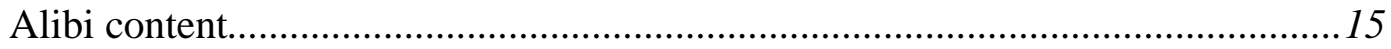

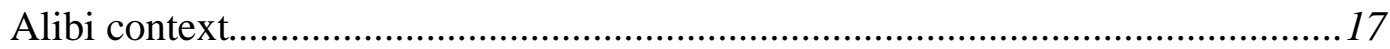

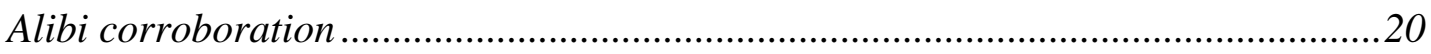

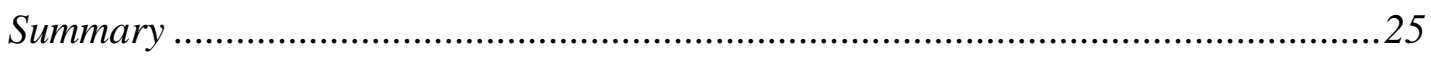

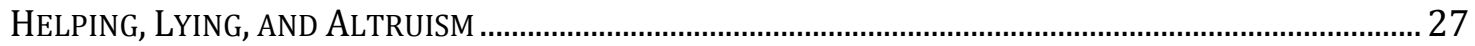

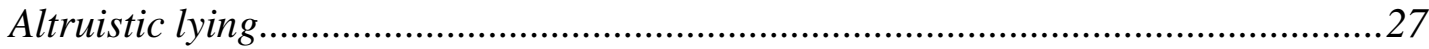

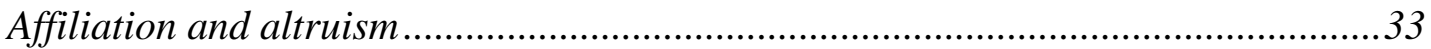

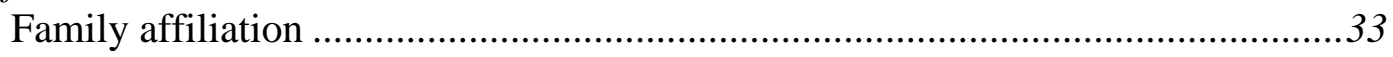

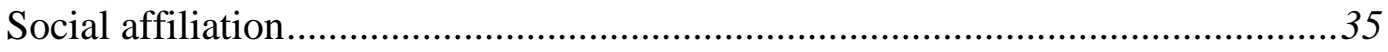

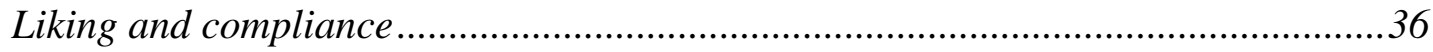

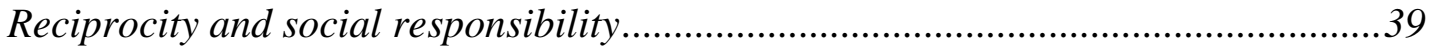

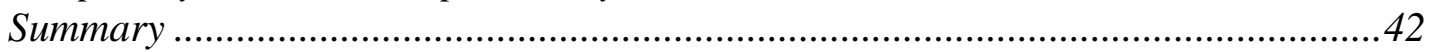

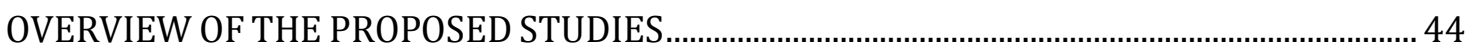

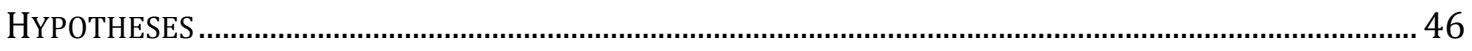

STUDY 1: EFFECT OF LIKING AND SOCIAL PRESSURE .........................................47

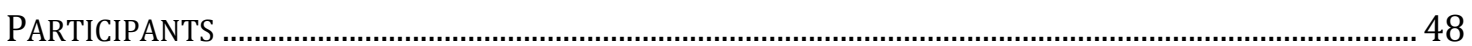

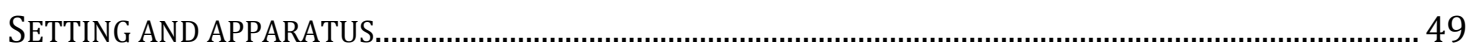

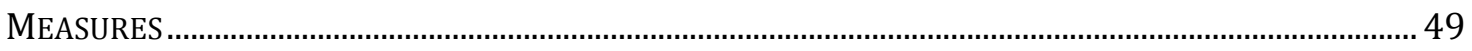

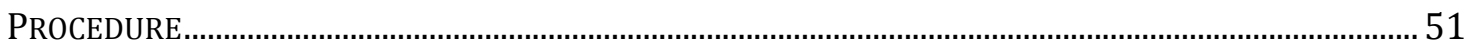

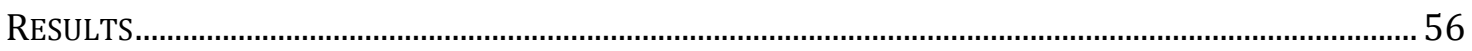

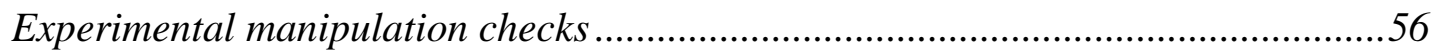

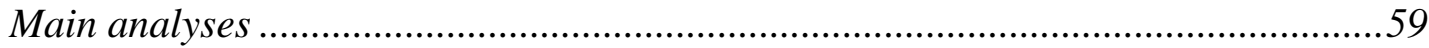

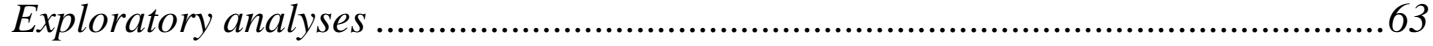

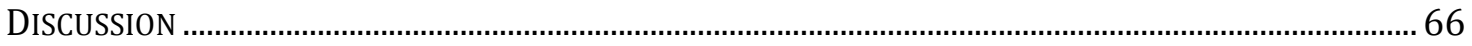

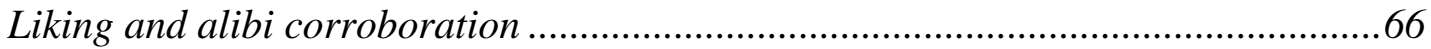

Compliance and alibi corroboration .....................................................................6 68

The role of memory ................................................................................................69

Reasons for lying and telling the truth............................................................... 71 


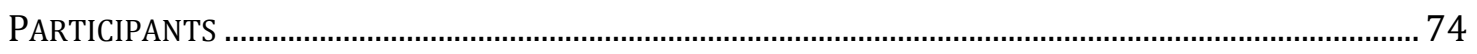

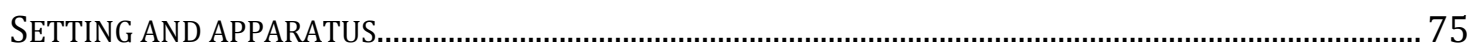

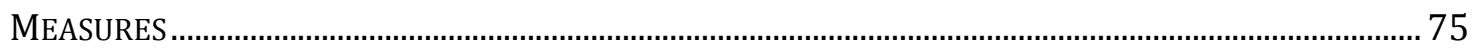

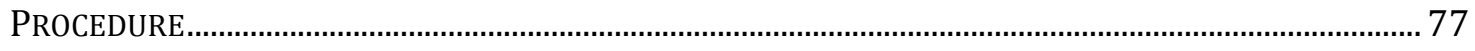

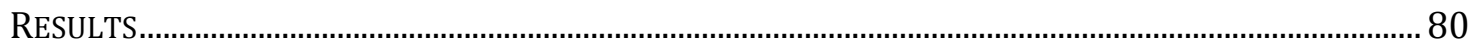

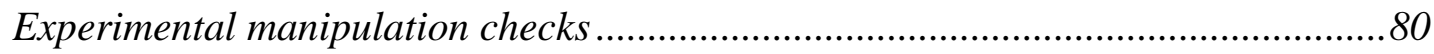

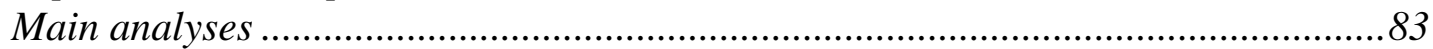

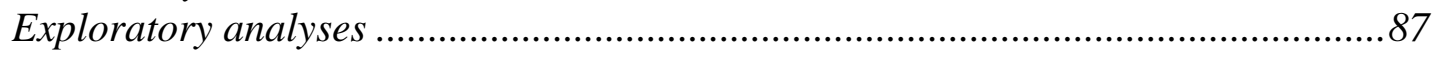

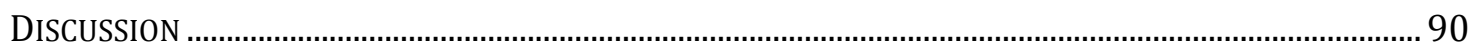

Prior help, reciprocity, and alibi corroboration .................................................. 91

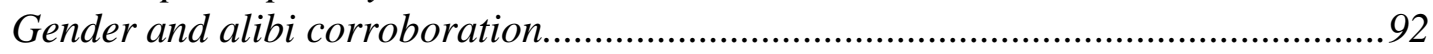

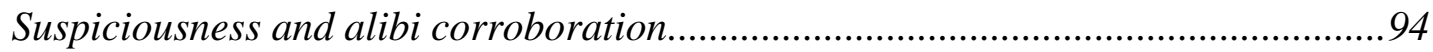

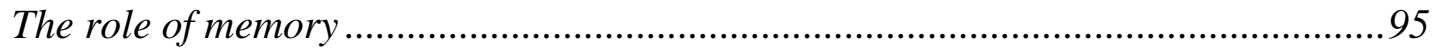

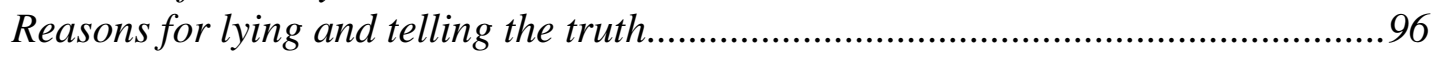

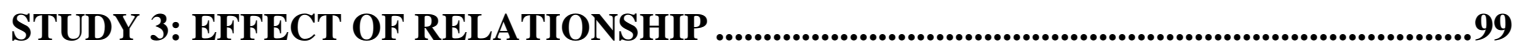

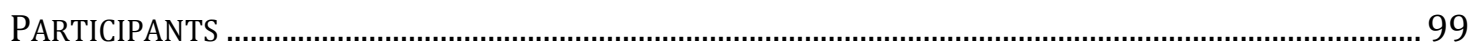

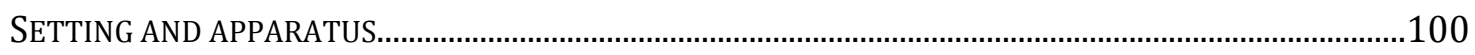

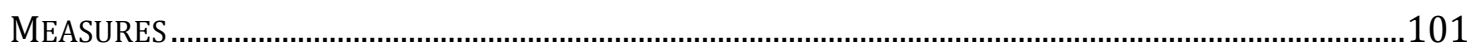

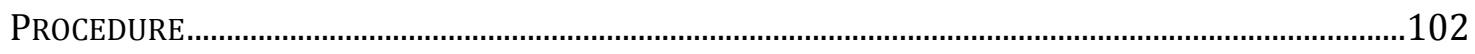

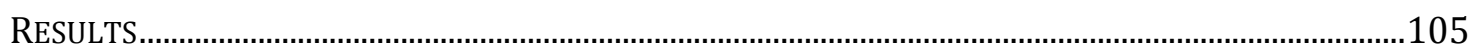

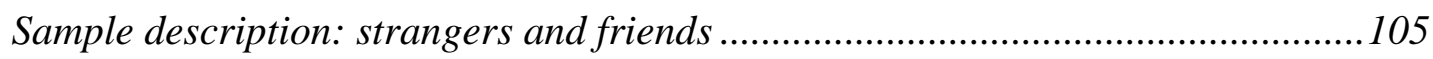

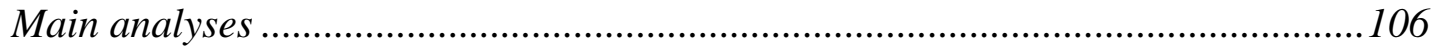

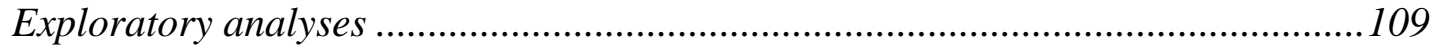

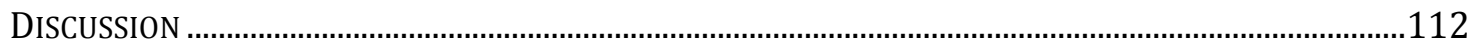

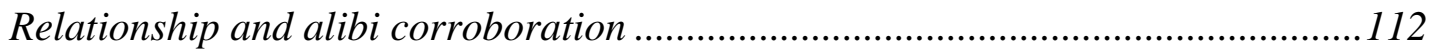

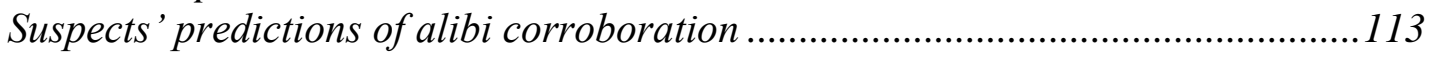

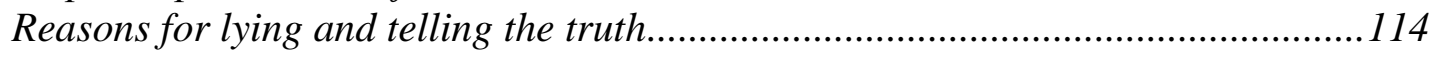

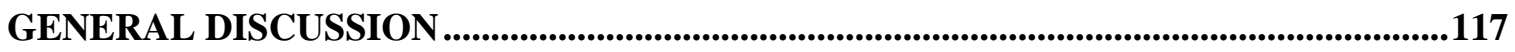

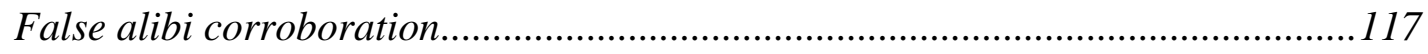

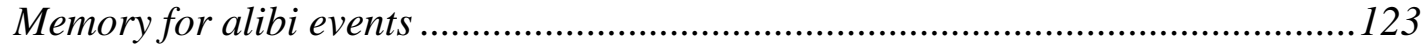

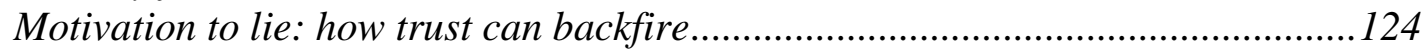

False alibi corroboration: not always an altruistic act ...................................... 125

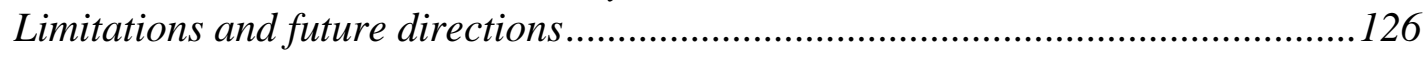

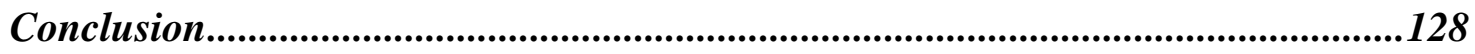

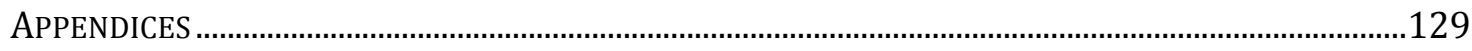

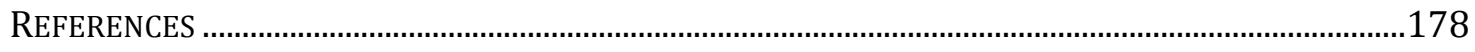




\section{List of Tables}

TABle 1. Alibi Corroboration Contingency TABle: Relationship CONDITION × REQUEST

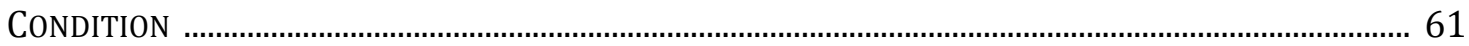

TABle 2. Alibi Corroboration Contingency TABle: HelP Condition × GENDER ........................ 85

TABle 3. Alibi Corroboration Contingency TABLE ....................................................................107 


\section{List of Figures}

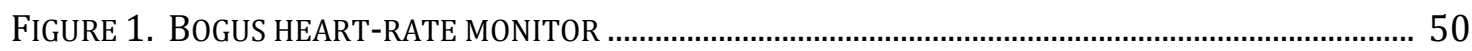

FiguRE 2. PERCENTAGE OF PARTICIPANTS WHO CORROBORATED THE CONFEDERATE'S FALSE ALIBI, BY

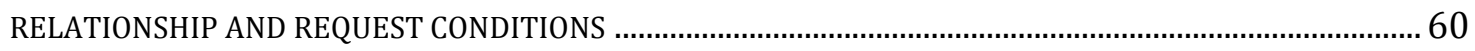

FIGURE 3. PERCENTAGE OF PARTICIPANTS WHO CORROBORATED THE CONFEDERATE'S FALSE ALIBI, BY

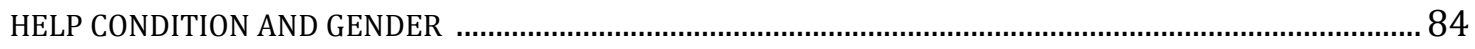

FIGURE 4. NUMBER OF ALIBI WITNESSES WHO CORROBORATED THE FALSE ALIBI, AS A FUNCTION OF RELATIONSHIP CONDITION AND CONFEDERATES' PREDICTIONS ......................................................... 


\section{List of Appendices}

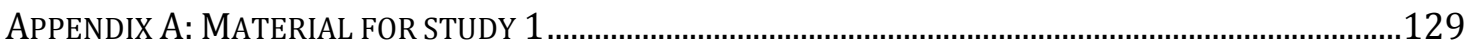

APPENDIX B: CODING MANUAL FOR DEBRIEFING QUESTIONNAIRE.......................................................137

APPENDIX C: PARTICIPANTS' EXPLANATIONS FOR REJECTION OR CORROBORATION OF THE ALIBI

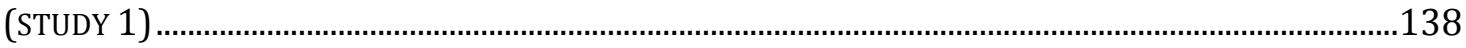

APPENDIX D: MATERIAL FOR STUDY 2

APPENDIX E: PARTICIPANTS' EXPLANATIONS FOR REJECTION OR CORROBORATION OF THE ALIBI

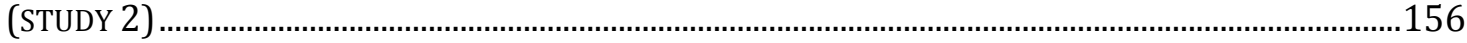

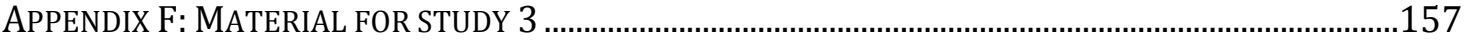

APPENDIX G: PARTICIPANTS' EXPLANATIONS FOR REJECTION OR CORROBORATION OF THE ALIBI

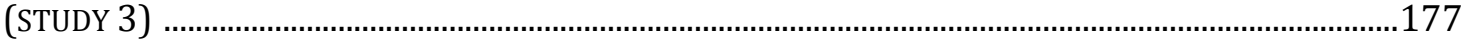




\section{Introduction and Literature Overview}

In 2014, the Innocence Project, a U.S.-based non-profit organization whose mandate is to free wrongly incarcerated individuals, helped exonerate their 316th innocent person (www.innocenceproject.org). In the 20 years of their existence they have fought to have DNA-containing biological evidence from likely cases of wrongful convictions retested to provide definitive exonerating evidence. State, national, and international-level organizations that have mandates similar to that of the Innocence Project are now widespread across the United States, Canada, Australia, New Zealand and the United Kingdom. The existence of wrongful convictions in these jurisdictions is now an irrefutable fact. A new National Registry of Exoneration published by American legal scholars currently reports over 1300 known exonerations in the United States alone since 1989, and an average incarceration period of just over 10 years for these exonerees. (www.exonerationregistry.org). However, since the actual ratio of rightful to wrongful convictions is nearly impossible to determine, and because most organizations advocating for the wrongly convicted have limited resources and therefore restrict their workload to cases involving particular types of crimes (e.g., homicides) or that include specific kinds

of evidence (e.g., DNA), the known number of wrongful convictions likely only represent the "tip of the iceberg" (Garrett, 2008, p.5).

Since cases of wrongful convictions began to surface in the 1990s (thanks to the emergence of advanced DNA testing technology), social scientists and legal scholars have examined exoneration cases in an effort to uncover the causes of such injustices. Topping the list of common contributing factors are mistaken eyewitness testimony, police or prosecutorial misconduct (including perjury from prosecution witnesses), faulty 
forensic evidence against the defendant, and false confessions (e.g., Connors, Lundregan, Miller, \& McEwen, 1996; Garrett, 2008; Wells et al., 1998). It is not surprising that these factors involve erroneous evidence in support of a defendant's guilt. However, in spite of the proverbial "innocent until proven guilty" criterion of our criminal justice system, a lack of evidence in support of a defendant's innocence may also contribute to increased judgements of guilt. Specifically, absent or weakly supported alibi evidence has been identified as a factor contributing to wrongful convictions (e.g., Burke \& Marion, 2012; Connors et al., 1996). An alibi is a statement or narrative that describes a defendant's actions and whereabouts at the time a crime occurred which, if true, would make it impossible that he or she is guilty. An alibi provider is the suspect in a case, or the person offering an alibi statement in response to an accusation. Thus, by definition, all innocent suspects or defendants should have an alibi. An alibi that is missing in a case could be due to either a suspect's complete lack of memory for what he or she was doing, and where, at the time of the crime; an alibi that is not investigated by the police or prosecution; or an alibi that was investigated but then is not presented at trial. A 'weak' alibi, on the other hand, is one that exists and may be presented at trial, but one that is not well supported by evidence according to investigators, judges and/or jurors. Alibi evidence is any kind of evidence corroborating the alibi (i.e., supporting its veracity), and can take the form of either physical evidence (e.g., a store receipt or phone record) or of alibi witnesses or corroborators (i.e., persons claiming to have knowledge of a defendant's whereabouts during the time of the crime and who confirm the veracity of the alibi). Even in the face of a mistaken eyewitness identification or a false confession, a true alibi that can be, and is, verified should protect an innocent person from a wrongful 
conviction. However, a true alibi is only effective if those who must evaluate it believe it.

The extent to which an alibi is believed depends on such factors as the type of corroborating evidence available, the relationship between an alibi witness and the suspect, the consistency of the alibi story, or even the content of the alibi itself (e.g., Allison, Mathews, \& Michael, 2012; Culhane \& Hosch, 2012; Olson \& Wells, 2004). For example, findings from social psychology research suggest that an alibi that is corroborated only by individuals who are closely related to the defendant tends to be judged with more skepticism than an alibi that is corroborated by individuals who do not share a personal relationship with the defendant, (e.g., Hosch, Culhane, Jolly, Chavez, \& Shaw, 2011). This kind of empirical evidence could help explain the outcome of wrongful conviction cases such as that of Dale Brison, who in 1990 was convicted of several counts of rape, kidnapping, assault, and weapons charges. The only evidence against Brison was an eyewitness identification (which turned out to be erroneous) and class evidence (i.e., evidence which identifies the suspect as belonging to the same group as the perpetrator because of a shared characteristic, such as blood type). Brison was really at home on the night of the crime, but his alibi was weak, as only his mother could corroborate his story (innocenceproject.org). After serving three and a half years of his 18-to-42-year sentence, DNA evidence excluded him as the perpetrator and he was finally released from prison.

Such 'weak' alibis have been observed as contributing factors in roughly $25 \%$ of wrongful conviction cases (e.g., Burke, Turtle, \& Olson, 2007; Connors et al., 1996). In these cases an alibi was presented at trial, but the jury did not believe it. However, as 
Dysart and Strange (2012) have argued, an ineffective alibi was likely present in most, if not all, wrongful conviction cases. It can be assumed that most suspects initially provided the police, their attorney, or the prosecutor the details of where they really were at the time of the crime, but their alibis were disbelieved by someone along the investigative process. As will be discussed in more detail below, a possible explanation for why this happens is the common assumption held by jurors and investigators that alibi evidence in the form of corroboration by witnesses who are close to a suspect or defendant (e.g., friends and family members) is not trustworthy because these individuals are motivated to lie (e.g., Hosch et al., 2011; Olson \& Wells, 2004). Thus, alibis that can only be corroborated by such witnesses, which unfortunately constitute the most common type of alibi provided by suspects (Burke \& Turtle, 2003; Olson \& Charman, 2012), are likely to be dismissed by investigators, prosecutors, and even defense attorneys.

This dissertation will examine 1) whether characteristics of the alibi provider, of the alibi witness, or of the interaction between the two can predict the likelihood that alibi witnesses will lie for a suspect by corroborating an alibi that they know is false, and 2) whether the assumption that the trustworthiness of an alibi depends on the closeness of the relationship between the alibi provider and the alibi corroborator is supported by behavioural data. It makes intuitive sense to predict that friends and family members of a suspect would be motivated to spare the latter the hardship of being the subject of a criminal investigation, criminal charges and judicial conviction. Evolutionary psychology would also predict that the closer an alibi witness is to an alibi provider, either biologically or socially, the more advantageous it would be for the witness to act altruistically toward the suspect (e.g., by lying for him or her, Hamilton, 1964; Trivers, 
1971). Finally, the social psychology literature on liking, compliance, and helping would suggest that individuals are more likely to comply with requests for help from others whom they like, and presumably this would include more friends and family members than strangers (e.g., Burger, Soroka, Gonzago, Murphy, \& Somervell, 2001). Although findings from previous research on alibi corroboration seem to support these predictions, virtually all studies thus far utilized self-reports to measure people's hypothetical willingness to corroborate a false alibi (i.e., lie) for suspects with whom they share varying degrees of relatedness (e.g., Hosch et al., 2011). The sole behavioural study investigating lying in an alibi corroboration context did not find that liking a suspect predicted false alibi corroboration (Marion \& Burke, 2013). Thus, further empirical work is needed to substantiate previous findings from self-report studies that the relationship between an alibi provider and alibi witness impacts the rate of deceitful corroboration.

Corroborating an alibi that one knows is false is a behaviour that can be viewed negatively (i.e., as a lie to an investigator, judge, or jury), or positively (i.e., helpful and altruistic toward a suspect). Thus, the existing literature on lying, altruism, and helping can provide a theoretical basis on which to make predictions about relational and situational factors that can affect the rate of false alibi corroboration. For example, social psychology research suggests that individuals exert more effort toward helping someone who depends on them if they were previously helped by that person than if they were not (e.g., Berkowitz \& Daniels, 1964). It is therefore possible that an alibi witness would be more likely to lie for a suspect if in the past the suspect has helped them in some way. Thus, in addition to a comprehensive review of alibi research, the following literature review will include a discussion of the psychological literatures on lying behaviour (in 
particular altruistic lying, or lying for the benefit of others), affiliation and altruism, liking and compliance, and reciprocity, as they relate to helping in the context of deceitful alibi corroboration.

\title{
Research Questions
}

Three studies examined factors that may impact the likelihood that an alibi witness would lie for a suspect by corroborating an alibi that they know is false. Six specific questions were asked:

1) Does the likelihood that an alibi witness will lie for a suspect depend on how much this witness likes the suspect? (Study 1)

2) Can a suspect increase the likelihood that an alibi witness will corroborate a false alibi by simply asking the witness to do so? (Study 1)

3) Is an alibi witness more likely to corroborate a suspect's false alibi when the suspect has previously helped the witness than when he or she has not? (Study 2)

4) Are women more or less likely to lie for one another than are men in a false alibi corroboration situation? (Study 2)

5) Is a friend of a suspect more likely to lie for him or her than a stranger? (Study 3)

\begin{abstract}
Alibis
Born out of the social and cognitive psychology literature on eyewitness evidence as well as from social scientists' interest in wrongful conviction cases, research on alibi evidence is relatively new, but growing steadily (Burke et al., 2007). Alibi corroboration evidence is similar in nature to eyewitness evidence, in the sense that in both types of
\end{abstract}


evidence, a witness testifies that the suspect was at a certain place, during a certain time (i.e., during the time of the crime). The main differences are that eyewitnesses place the suspect at the scene of the crime whereas alibi witnesses place him or her anywhere but at the scene of the crime, and that eyewitnesses usually do not know the suspect whereas alibi witnesses often do (Burke \& Turtle, 2003). Despite the similarities, there is a relative lack of research on alibi evidence. For example, a keyword search on PsycINFO for "eyewitness" or "eyewitness evidence" yields 2523 hits, whereas a search for "alibi witness" or "alibi evidence" produces 51.

The research on alibi evidence that does exist can be separated into three different areas, which coincide with separate phases through which alibi evidence moves within the judicial process: alibi generation, alibi corroboration, and alibi evaluation (this classification is based in part on a model developed by Olson (2002, as cited in Burke et al., 2007) and elaborated by Burke et al., 2007). During the alibi generation phase, a suspect generates a memory of where he or she was and what he or she was doing at the time that a crime occurred. During the alibi corroboration phase, the suspect attempts to find either physical evidence that can corroborate his or her alibi or one or several individuals who can support the veracity of the alibi. Finally, during the alibi evaluation phase, the police, judge, or jury makes a decision about whether the alibi is likely to be true or false. The research developments made in these three literatures will be discussed in this section.

Alibi generation. When an individual is approached by the police and is informed that he or she is suspected of having committed a crime, a likely scenario, especially if the suspect is innocent, is that he or she will provide the police with a reason 
why it is impossible that he or she is guilty: the suspect was somewhere else during the time of the crime. According to a sample of police officers surveyed by Dysart and Strange (2012) criminal suspects spontaneously provide an alibi in $62 \%$ of cases. This initial alibi can either be a false alibi (if the suspect is guilty and wishes to deceive the police), a true alibi (if the suspect is innocent and remembers where he or she was at the time of the crime), or a mistaken alibi (if the suspect is innocent but misremembers where he or she was at the time of the crime, Burke et al., 2007). Thus, an alibi witness can be mistaken, just as an eyewitness can be. The accuracy and reliability of an alibi provider (and alibi witnesses) is dependent on autobiographical memory and is vulnerable to decay just as much as the accuracy and reliability of an eyewitness' testimony. In fact, alibi evidence may be even more vulnerable to the fallibility of human memory than eyewitness evidence since alibi providers and witnesses are required to remember events that were likely mundane and insignificant. Whereas eyewitnesses may immediately realize that they are witnessing a crime, it is unlikely that innocent suspects (or alibi witnesses) are aware that a crime is occurring elsewhere at a certain time and that they should therefore make note of their whereabouts and activities in the event that they may become a suspect. Thus, it is possible that the events recalled when generating an alibi are not encoded as well as the events remembered by an eyewitness, and are therefore more likely to be misremembered or not remembered at all. This may explain, for example, why alibi providers often rely on scripts (i.e., routine activities) as opposed to actual memory traces in order to remember events, especially those from the distant past (Olson \& Charman, 2012), and why a subset of suspects do not spontaneously give police investigators an alibi (Dysart \& Strange, 2012). 
Research on alibi generation is limited, with just a few recently published studies offering data on the accuracy of spontaneously generated alibis and on the likely characteristics of true and false alibis. In one such recent study, Olson and Charman (2012) asked participants to generate alibis for either a time in their recent past (e.g., 3 days prior to the study) or a time in their distant past (e.g., 6 to 14 weeks prior), by relying only on their memory. They then gave participants 48 hours to investigate their alibi and try to collect corroborating evidence to support it. Most participants (88\%) were able to spontaneously generate an alibi, but $8 \%$ of participants were still unable to remember their whereabouts and activities at the given time even after the 48-hr investigation period. In addition, $36 \%$ of the initial alibis required a change after the investigation, either in the alibi content itself (12\%) or in the corroborating evidence that participants initially thought they could offer (24\%). Not surprisingly, more alibis from the distant past needed to be changed than alibis from the recent past. In a similar study by Strange, Dysart, and Loftus (2014) in which participants were given one week to investigate and re-tell their alibi, only $53 \%$ of the details provided were consistent over the 7-day period. The inconsistencies included changes in activities during the time in question (32\% of alibis), the timing of their activities (44\%), the individuals present (23\%), the location (19\%), and their whereabouts before and after the critical alibi period (33\% and $40 \%$, respectively).

Entire changes in alibi stories over time, however, appear to occur much less frequently. In a third study using a paradigm similar to Olson and Charman (2012), Culhane and colleagues (2013, study 1) asked participants to report a true or a false statement about their activities and whereabouts on an evening either 5 or 12 days before, 
and repeat this statement two days later. Complete changes in stories were rare, but occurred more often for false alibis $(12 \%)$ than true alibis $(4 \%)$. The reason for the complete changes in false alibis were not described but one could speculate that participants either forgot their first lie, or thought of another story which they believed consisted of a better lie. Thus, a complete change may be indicative of deception, however the finding that substantial details of true alibis often change over time as well (e.g., Olson \& Charman 2012; Strange et al., 2014) may be cause for concern since, as will be discussed in the next section, alibis that are changed are likely to be perceived as false (e.g., Dysart \& Strange, 2012).

Allison, Michael, Mathews, and Overman (2011) speculated that guilty suspects who provide a false alibi likely rehearse an alibi (that reflect actual experiences) before being interviewed by police, whereas innocent suspects are more likely to be forced to spontaneously provide an alibi, without having much time to think about their whereabouts or consult external resources (e.g., cell phone, day planner). To try to identify unique characteristics of these rehearsed alibis, they analyzed and compared the audio-recorded narratives of (presumably true) alibis provided by participants who had either been given 3 minutes to think about their whereabouts for a certain time period or were unprepared and asked to spontaneously provide their alibi. The only differences between the two narratives were that prepared alibis contained more vocabulary indicating confidence in a statement (i.e., "definitely", "for sure”, "no doubt”) and fewer pauses during the beginning of the narrative than unprepared alibis. There were no differences in the use of vocabulary suggesting doubt, logical connections, temporal connections, personal pronouns, elaborations, or spatial order. Thus, as these findings 
suggest and as the literature on deception detection would predict (e.g., Vrij, 2008), it may be difficult to identify false from true alibi narratives using speech cues. In fact, when asked to judge the veracity of true and false alibis, undergraduate participants do not fare well, with an accuracy rate of only 43\% (Culhane et al., 2013, study 2). It may be a good thing, then, that people seem to rely on other content, context, and evidentiary cues when evaluating the accuracy and reliability of alibi evidence.

Alibi evaluation. The vast majority of the published literature on alibi evidence has focussed on how it is evaluated during the course of an investigation or in the courtroom. One of the most robust findings to date is that alibis that are corroborated by individuals who are closely related to the suspect are perceived as less believable than alibis that are corroborated by individuals who are distantly related or are strangers to the suspect (e.g., Culhane \& Hosch, 2004; Hosch et al., 2011; Olson \& Well, 2004). This finding was first revealed, almost by chance, in a mock-juror study by Lindsay, Lim, Marando, and Cully (1986, study 2). They examined whether a lack of consensus between eyewitnesses testifying for the prosecution and 'eyewitnesses' testifying for the defense (i.e., alibi witnesses) would decrease the strength of the prosecution's case compared to cases in which only prosecution eyewitnesses testified. The introduction of alibi evidence to a case in which an eyewitness identified the defendant as the perpetrator reduced the rate of guilty verdicts only when the alibi witness was a stranger. When the alibi witness was a family member, the guilty verdicts were just as high as when no alibi witness testified. Although the intended focus of the study was on eyewitness testimony, their findings suggested that the strength of alibi evidence, in terms of its ability to cast doubt on the guilt of the suspect, may depend on the identity of the alibi corroborator. 
Subsequent studies attempted to further investigate the relationship between the persuasive strength of alibi evidence and the type of corroborating evidence (or witness) offered by the alibi provider. In another mock-juror study, Culhane and Hosch (2004) compared the effect of the testimony from an alibi witness who shares a close social relationship with a defendant but is not biologically related (e.g., the defendant's girlfriend) with that of the testimony from an alibi witness who does not share a close relationship with the defendant, but is nonetheless not a stranger (e.g., the defendant's neighbour). Similar to the findings from Lindsay et al. (1986, study 2), in comparison to when the defense did not present any alibi evidence, testimony that the defendant was at home during the time of the crime did not decrease guilty verdicts when this information was provided by the girlfriend, but did when presented by the neighbour, again suggesting that corroborating alibi evidence from a person sharing a close relationship with the suspect does not increase the strength of the defense's case.

Physical vs. witness evidence. Olson and Wells (2004) investigated how different types of physical evidence, in addition to different types of 'person' evidence (i.e., alibi witnesses) could affect the evaluation of alibis. With the goal of creating a taxonomy of alibis based on the strength of the evidence supporting it, they manipulated both the presence and type of physical evidence (based on its ease of fabrication) and person evidence (based on the relationship between the alibi witness and the defendant) that corroborated a suspect's alibi in a series of fictitious crime scenarios. Mock investigators gave higher believability ratings for alibis that were supported by any kind of physical evidence (regardless of ease of fabrication) than for alibis that could not be corroborated by physical evidence, with the exception of when a stranger corroborated the alibi (in 
which case the addition of any kind of physical evidence did not increase believability ratings). When there was any kind of corroborating physical evidence, neither the presence nor the type of person evidence increased the believability of the alibis. The person evidence manipulation, however, did affect the believability of alibis when there was no corroborating physical evidence: under this condition, mock investigators gave higher believability ratings for alibis that were corroborated by strangers than for alibis that were either corroborated by no one or corroborated by what they termed 'motivated' others (individuals who would be motivated to lie for a defendant, such as friends and family members). A fourth type of alibi witness, 'non-motivated familiar others', or persons who know a defendant but would not be motivated to lie for them (e.g., the teller at the defendant's bank), also made alibis more believable than alibis which no one corroborated, but made them no more or less believable than alibis corroborated by strangers or by motivated others. Olson and Wells' (2004) findings are also consistent with those of Lindsay et al. (1986), Culhane and Hosch (2004) and several others since (e.g., Dahl \& Price, 2012) in that alibis corroborated by a motivated witness (i.e., a friend or a family member) are not significantly more believable than alibis that are offered by a defendant but are not corroborated.

Although most studies on alibi evaluation use college students as mock jurors or mock investigators, recent evidence confirms that American and Canadian police officers also rely on the type of corroborating evidence when evaluating the believability of an alibi. Officers surveyed by Dysart and Strange (2012) reported that the most believable alibis are those for which there is physical evidence, corroboration from multiple witnesses, corroboration from non-motivated witnesses, or a mix of person and physical 
evidence. Thus, the finding that alibis corroborated by physical evidence and/or alibi witnesses who are not closely related to the suspect are perceived as more believable than alibis corroborated only by adult witnesses who are related to a suspect has proved robust and has been replicated many times with different study designs and different samples (e.g., Allison \& Brimacombe, 2010; Allison et al., 2012; Hosch et al., 2011). One exception was recently noted, however, for situations in which the corroborating person evidence comes from a child alibi witness: Results from Dahl and Price (2012) suggest that when the corroborating witness is a child, the relationship that he or she shares with an alibi provider does not seem to influence perceived believability of the alibi. For example whereas the corroboration from an adult son of the suspect does not decrease perceived guilt, the corroboration from a child who is the suspect's son, on the other hand, does (Dahl \& Price, 2012). The authors speculated that child witnesses might be perceived as more honest than adult witnesses, and thus less likely to lie, even for a relative.

Degree of relatedness. Instead of simply comparing the effect of relationship type (e.g., family member vs. friend vs. stranger) on the believability of an alibi, Hosch et al. (2011) investigated whether the degree of relatedness between an alibi provider and an alibi corroborator had an effect on the perceived credibility of the alibi witness. The degree of relatedness was manipulated for three different types of relationships: biological, affinal (relation through marriage, i.e., 'in-laws'), and social. For example, a brother or sister is more closely related to a suspect than a cousin (biological relationship); a husband or wife is more closely related than a sister- or brother-in-law (affinal relationship); and a best friend is more closely related than a neighbour (social 
relationship). As expected, Hosch and his colleagues found a main effect of relationship type, where biologically related alibi witnesses were rated as less credible than affinally related witnesses, who were in turn rated as less credible than socially related witnesses. In addition, they found a linear relationship between credibility and the degree of relatedness within each of the three relationship types, where the closer the relationship, the less the alibi witness was thought to be credible. For example, within biological relationships, participants estimated that an identical twin would lie $85 \%$ of the time, a half-sister $54 \%$ of the time, and a second cousin $32 \%$ of the time.

Alibi content. Having established the link between alibi evaluation and the alibi provider-corroborator relationship, social psychologists turned to identifying other factors that might influence the believability of an alibi, namely the content of the alibi story and the context in which the alibi is presented. In a mock juror study by Allison et al. (2012), participants gave higher alibi believability ratings, lower suspect guilt ratings, and higher alibi witness credibility ratings when they read about a suspect's alibi that included watching an adult movie than when it included watching a regular movie. Interestingly, suspects who provided the salacious alibi were judged by mock jurors to be more trustworthy, honest, and open, and less suspicious, than suspects who provided the nonsalacious alibi (however, see Jung, Allison, \& Bohn, 2014). It is possible that mock jurors reasoned that if a guilty suspect had to fabricate an alibi, he would probably not purposefully portray himself as a morally undesirable individual (Allison et al., 2012). In another study by Maeder and Dempsey (2013), the degree to which an alibi provided by a murder suspect was female-stereotypic (e.g., shopping for skin care products) or malestereotypic (e.g., shopping for tools) indirectly influenced participants' rating of alibi 
believability and defendant credibility by impacting participants' perception of the femininity of the defendant: Defendants who were perceived as more feminine were more credible and their alibi more believable than defendants who were perceived as more masculine. It was speculated that since aggressive behaviour is often associated with masculinity, that a masculine defendant would be more likely to have committed an aggressive crime than a feminine defendant. Although further research is needed, the content of an alibi narrative seems a promising new factor to investigate within research on alibi evidence and evaluation.

Another factor that has appeared in recently published research on alibi evaluation is the level of consistency of an alibi over time. Despite the fact that initially-generated alibis are often mistaken and need to be modified (e.g., Olson \& Charman, 2012) findings from alibi evaluation studies overwhelmingly suggest that changed alibis are not believed. For example, $90 \%$ of participants in Culhane et al.'s (2008) study reported believing that an alibi provider who changes his or her alibi is most likely lying. Police officers surveyed by Dysart and Strange (2012) reported believing that $81 \%$ of suspects who change their alibi do so because they initially lied (as opposed to being mistaken). Culhane and Hosch (2012) compared participants' evaluations of alibis that remained consistent over time with those that either changed and became stronger (by increasing the strength of the corroborating evidence) or those that changed and became weaker (by decreasing the strength of the corroborating evidence). They found that, although alibis that changed by increasing in strength were slightly more believable than alibis that decreased in strength, both types of changes significantly reduced the believability of the alibis and increased ratings of suspect guilt in comparison with consistent alibis. The 
question of whether these credibility judgements reflect a true difference in the consistency of true and false alibi statements, however, has yet to be confidently answered. As stated earlier, when analyzing both true and false alibis before and after a two-day period, Culhane et al. (2012) found that the contents of false alibi stories were three times more likely to have changed over time than the content of true alibi stories. However, an opposite pattern emerged in a study by Granhag, Strömwall, and Jonsson (2003): Pairs of participants, who either had or pretended they had gone to lunch together, were interviewed about their true or made-up lunch date. Members of each pair were interviewed twice, individually, and the videos of their interviews were subsequently evaluated by a different group of participants. The interviews of those lying were rated as more consistent than the interviews of those telling the truth, both over time within the same individual and within the pair at both recall times. The authors of this study suggest that liars may simply repeat what they have rehearsed, whereas truth-tellers are reconstructing a true event from memory, making the latter more vulnerable to inconsistencies between and within individuals. Thus, this suggests that the alibis that are most likely to be believed may be the ones that are most likely to be false. One possibility could be that false alibi stories are more likely to change over time compared to true alibis, as seen in Culhane et al. (2012), but that the details provided within false alibis are less likely to change over time compared to true alibis (as per Granhag and colleagues' findings), although further research would be needed to explore this idea.

Alibi context. The evaluation of an alibi may also depend on when it is evaluated. For example, Sommers and Douglass (2007) manipulated the context in which college students were asked to evaluate an alibi: at the investigation stage (i.e., mock investigator 
context), or at the trial stage (i.e., mock juror context). They found that an alibi that was corroborated by a family member increased the believability of the suspect's alibi compared to an uncorroborated alibi, but only in the context of an investigation. When asked to evaluate an alibi in a trial context, the authors proposed, participants might have reasoned that if the case proceeded to trial despite an initial police investigation, then the alibi must not have been convincing, regardless of the corroborating evidence. In the investigative context, however, participants may have been more willing to give the corroborating witness the benefit of the doubt (Sommers \& Douglass, 2007). It is possible, however, that these findings may not hold with a sample of actual investigators, since interrogations of alibi providers (i.e., suspects) are usually conducted only once guilt is presumed (see Kassin, Goldstein, \& Savitsky, 2003), and since a thorough investigation of an alibi is not always conducted before an arrest is made (Dysart \& Strange, 2012).

Finally, there is some limited research available on how the presence of other kinds of evidence, such as eyewitness evidence and prior convictions, influences the evaluation of alibi evidence. Findings from mock investigator studies by Dahl, Brimacombe, and Lindsay (2009) and Price and Dahl (2014) suggest that, as might be expected, a suspect's alibi is perceived as less credible when an eyewitness identifies the suspect as the perpetrator than when an eyewitness fails to identify him or her from a lineup. In another study, Allison and Brimacombe (2010) tested the impact of an alibi provider's prior conviction on alibi believability and guilt ratings and found that a suspect who had a prior conviction for perjury was believed less than a suspect who had a prior conviction for a different crime unrelated to the current charge. Allison et al. (2012) also 
found that mock jurors gave higher suspect guilt ratings when the alibi corroborator had a criminal record than when he did not.

A general conclusion that can be drawn from the research on alibi evaluation is that people are generally very sceptical of alibi evidence. For example, Olson and Wells (2004) found that the highest average alibi believability score given on a 1 to 10 Likert scale was 7.4, which was for alibis corroborated with both physical evidence that was difficult to fabricate (e.g., a date and time-stamped surveillance video) and an alibi witness who was a stranger to the suspect. Furthermore, police officers believe that most suspects (78\%) have lied to them by providing a false alibi during the course of an investigation (Dysart \& Strange, 2012). Participants in Culhane et al.'s (2008) study reported believing that roughly $50 \%$ of alibi witness who are friends or family members of a suspect lie in court, and similarly, actual police officers believe that 'motivated' witnesses lie between 38\% and 53\% of the time (Dysart \& Strange, 2012). Officers also believe that even acquaintances and strangers lie $26 \%$ and $13 \%$ of the time, respectively, by corroborating a false alibi, either because they distrust the police, fear or are under pressure from the suspect, have been promised some type of reward, or "feel bad" for the suspect. With a general lack of objective knowledge as to the guilt or innocence of criminal suspects, however, it is difficult to determine the accuracy and reasonableness of these judgements.

On a more optimistic note, Olson and Wells (2010) have recently identified a potential way to reduce individuals' scepticism of alibi evidence: They asked participants to either generate their own alibi first and then evaluate a suspect's alibi, or 
perform these two tasks in the reverse order. They found that those who generated their own alibi before evaluating someone else's alibi gave it higher ratings of believability than participants who evaluated the alibi first, and then generated their own, a phenomenon that they termed the 'alibi-generation effect'. The robustness and generalizability of this effect remains to be confirmed with further study, but this may be a potential way to shift jurors' perceptions of what represents a 'strong' or 'weak' alibi by giving them first-hand experience in the potentially difficult task of generating an ironclad alibi.

Alibi corroboration. The fact that alibis that are corroborated by friends and family members are believed less than alibis that are corroborated by strangers or by physical evidence is of concern only if this type of corroborating evidence is not diagnostic of suspect guilt (or alibi truth). In other words, if innocent suspects are just as likely as guilty suspects to offer 'weak' evidence in support of their alibi, and if 'strong' evidence in support of an alibi is equally rare for guilty and innocent suspects, then the reliance on person and physical evidence in evaluating alibis may not be justified.

In reviewing a large sample of court cases in which alibi evidence was presented, Burke and Turtle (2003) observed that in approximately 86\% of the Canadian cases and $68 \%$ of the American cases an alibi witness testified and corroborated the alibi. All but two of the corroborating witnesses in the cases analysed by Burke and Turtle (or more than $98 \%$ ) were friends, family members, or co-workers of the accused (i.e., nonstrangers). Additionally, in only $2 \%$ of the Canadian cases and $14 \%$ of the American 
cases did the defendant offer physical evidence to support his or her alibi ${ }^{1}$. Although it is impossible to discriminate between the innocent suspects and the guilty ones when analyzing real cases, the data gathered by Burke and Turtle (2003) do suggest that alibi evidence that is considered strong by investigators and potential jurors (i.e., physical evidence and stranger alibi witnesses) is quite rare in actual court cases.

The prevalence of different kinds of corroborating alibi evidence for innocent and guilty suspects has been estimated by using research paradigms in which participants are asked to either imagine, or actually collect, evidence they think they could offer for true and false alibis. For example, Culhane, Hosch, and Kehn (2008), asked college students to imagine that they were suspected of having committed a crime that occurred at $9 \mathrm{pm}$ two days prior to the study session. They were then asked whether they thought they could find an alibi witness who could vouch for their actual whereabouts, and if so, what relationship they have to this individual. A large majority of participants (88\%) thought they could find an alibi corroborator, but most of the alibi witnesses listed (84\%) could be classified as motivated others (e.g., a parent, sibling, or friend). When asked whether they thought they could find or produce true physical evidence to corroborate their evidence, only $29 \%$ said yes, and of the pieces of evidence offered by these students, $59 \%$ were relatively weak pieces that could easily have been fabricated (or 'borrowed') such as receipts or ticket stubs. Going one step further than Culhane et al. (2008), several

\footnotetext{
1 This is in contrast to the estimate provided by U.S. and Canadian police officers surveyed by Dysart and Strange (2012), who thought that physical evidence was offered in $24 \%$ of cases. However, only $49 \%$ of these same officers reported that a thorough investigation of a suspect's alibi is always done before an arrest is made, which could explain why physical alibi evidence is so seldom presented at trial even though it may have been offered by the suspect beforehand.
} 
researchers (Olson \& Charman, 2012; Strange et al., 2014, describe in a previous section) have actually asked participants to investigate their alibis over the course of several days and to report back to the lab with any corroborating evidence that they could find. In these studies, between thirteen and 43 percent of all true alibis generated could not be corroborated by any kind of evidence, either person or physical, even after up to one week of investigation. In Olson and Charman (2012), 57\% of all alibis were corroborated by person evidence only (i.e., witnesses), $98 \%$ of whom were friends or family members. Similarly, in the study by Strange and colleagues (2014) $21 \%$ of alibis provided were corroborated only by witnesses, all but one of whom was a friend or family member. Surprisingly, in both studies, between 30 and $36 \%$ of alibis were corroborated by some kind of physical evidence, which is a high percentage in relation to the estimates provided by police officers (Dysart \& Strange, 2012) and court cases (Burke \& Turtle, 2003). However, most of the physical evidence offered by research participants was weak (64-70\%) and could easily be fabricated (e.g., a personal diary, a CD that was allegedly burned during the time of the crime, or a cash receipt, Olson \& Charman, 2012; Strange et al., 2014). Thus, when it comes to true alibis, the overwhelming majority of person evidence that is available to corroborate the alibis are statements provided by those who are assumed to be 'motivated' to lie: friends and family members of the suspect.

Obtaining a valid estimate of the frequency with which guilty suspects can obtain person and physical evidence to corroborate a false alibi is more difficult. Culhane et al. (2008) attempted to do so by also asking participants whether they thought they could find a person who would corroborate a false alibi in the event that they were suspected of 
committing a crime, and if so, who that person would be. This time, $61 \%$ thought they could obtain an alibi witness, but again, a large majority (97\%) of the proposed potential witnesses were friends or family members. Although this percentage of 'motivated' alibi witnesses available to corroborate hypothetical false alibis is higher than the estimated percentage of 'motivated' alibi witnesses available for hypothetical true alibis (84\%), it is highly similar to the percentage of 'motivated' witnesses available to corroborate true alibis (98\%), according to participants in Olson and Charman (2012). In addition, when Culhane and colleagues (2013, study 1) in their alibi generation study asked participants to actually provide corroborating evidence for both true and false alibis, false alibis were more likely to be corroborated by friends $(60 \%)$ than true alibis $(40 \%)$, whereas both types of alibis were equally likely to be corroborated by family members, acquaintances, co-workers, and strangers. When it comes to gathering physical evidence for a false alibi, similar to cases of true alibis, 34\% of Culhane and colleague's (2008) participants reported believing that they could obtain such evidence, but again most (80\%) pieces of (hypothetical) evidence offered were the kind that could easily be fabricated or found such as receipts and ticket stubs. Additionally, when asked to actually provide physical evidence to support true or false alibis, participants who offered false alibis were just as likely to provide physical evidence as those who offered true alibis (roughly $16 \%$ across both alibi types, Culhane et al., 2013, study 1). Thus far, the availability of physical evidence and stranger witness evidence in support of an alibi does not seem to have good diagnostic value for suspect guilt.

Another way to estimate how readily suspects could corroborate a false alibi is to ask people how willing they would be to lie for others. In Hosch et al.'s (2011) study on 
the degrees of relatedness between an alibi provider and an alibi corroborator (within biological, affinal, and social relationships, as described earlier), participants were asked to report on their willingness to lie in court for different individuals by corroborating a false alibi. Other details surrounding the hypothetical scenario, such as the crime committed and evidence again the suspect, were not specified. Participants were most willing to lie for biologically related defendants, followed by affinally related defendants, and finally were least willing to lie for socially related defendants. There were also linear relationships between willingness to lie and the degree of relatedness within each of the three relationship types. For example, $82 \%$ of participants reported being willing to lie for their spouse whereas only $45 \%$ were willing to lie for their sister-in-law, and $22 \%$ of participants reported being willing to lie for a co-worker whereas only $9 \%$ were willing to lie for a familiar stranger. It would therefore seem reasonable to predict that strangers and acquaintances of a suspect would be less likely to corroborate a suspect's false alibi than friends and family members of the suspect. However, there is sometimes a discrepancy between how people say they would act and how they actually act in a certain situation (e.g., Kraus, 1995).

We recently conducted a study to determine how likely individuals would be to lie for another by corroborating an alibi that they know is false (Marion \& Burke, 2013). Using a new experimental paradigm, participants were given the opportunity to either lie or tell the truth about a confederate's whereabouts. While a participant and confederate worked together on filler tasks, the confederate briefly left the testing room under the pretense of needing to use the restroom. After the confederate returned, the experimenter informed the pair that a theft had occurred in an office nearby and asked them if they had 
seen anyone enter the adjacent room. The confederate, in the presence of the participant, provided a false alibi by stating that she was in the testing room with the participant during the duration of the tasks and therefore had not seen anyone. Minutes later, when the participant was alone, the experimenter asked the participant to confirm the confederate's alibi. Two factors were manipulated in this study: how much the participant liked the confederate, and how suspicious the confederate's actions appeared to be. It was expected that those who were in a liking condition (manipulated by increasing their perceived similarity to, and the time they spent interacting with, the confederate) would be more likely to lie for the confederate than those who believed they were dissimilar to, and did not spend time with, the confederate. However, no such difference was found, and approximately $23 \%$ of participants in both conditions lied for the confederate. The suspiciousness of the confederate's actions, however, did have an impact on corroboration rates: participants were much less likely to corroborate the false alibi when they had previously seen the confederate handling money, the purported object of the theft, than when they had not (10\% vs. 36\%). This suggests that the closeness between an alibi witness and an alibi provider (at least as measured by liking) is not a good indicator of the likelihood that a witness will lie for a suspect. It does suggest, however, that other situational factors relating to the crime scenario (e.g., how suspicious the suspect seems) may be better predictors of when a witness is likely to corroborate a false alibi.

Summary. From the time an alibi is initially generated during a criminal investigation, to the time it is heard in court as testimony, alibis are being evaluated, and a judgement is being made as to whether the suspect or defendant is likely lying or not. 
The literature on alibi evaluation has mostly focussed on the effect of the relationship between alibi believability (and the suspect's likelihood of guilt) and the type of corroborating evidence that exists, including the relationship shared between the suspect and potential alibi witnesses. Findings from studies on this topic all point to the same conclusion: if an alibi is corroborated by a witness who is closely related to the alibi provider, whether biologically, affinally, or socially, it is unlikely to contribute much to the strength of a defendant's case. This phenomenon seems to be explained by the assumption held by investigators, mock investigator, and mock jurors alike that individuals who are closely related are more likely to lie for one another than are those who are distantly related or unrelated. People not only mistrust testimony from alibi witnesses who are closely related to a defendant, but they also expect that their friends and family would lie for them in a similar situation and report being willing to themselves lie in court in the event that their friends and family members would be suspected of committing a crime. However, despite these assumptions and expectations, the type of alibi evidence (i.e., person vs. physical) and the type of alibi witness (i.e., related vs. unrelated witnesses) that innocent suspects are able to provide in support of their alibis tend to be very similar to the kind of evidence provided by guilty suspects. Additionally, the (albeit limited) empirical evidence on the rate of false alibi corroboration suggests that the closeness between alibi witnesses and alibi providers may not affect the likelihood that witnesses will lie. Thus, social scientists may benefit from trying to identify other factors that could affect whether a witness will lie by corroborating a false alibi. One way to identify potential influential factors is to look to different but related psychological principles. 


\section{Helping, Lying, and Altruism}

If we are to investigate which alibi witnesses are more likely to lie and when, it is useful to start by placing the behaviour in a broader context. In essence, a witness knowingly corroborating a false alibi is lying, usually to an authority figure, to help a third party. Typically, there is no obvious direct benefit for the witness him or herself; in fact lying to authority carries risks in the form of social or legal sanctions if caught (e.g., being perceived as unreliable or being charged with obstruction of justice). Thus, knowingly corroborating a false alibi can be regarded as an altruistic lie. The expectation that alibi witnesses who are closely related to the alibi provider are likely to lie for them at a higher rate than alibi witnesses who are not can be explained by research on altruism. Hamilton's (1964) kin selection theory and Trivers' (1971) reciprocal altruism theory would predict that altruistic behaviour is more likely to occur between individuals who are biologically and socially close to one another, respectively. The tendency to help another in response to an implicit or explicit request (i.e., compliance) can be even more likely if liking is increased (Cialdini \& Trost, 1998), or if a sense of social responsibility is instilled, for example by responding to a previous act of helping (e.g., Berkowitz \& Daniels, 1964). The following section briefly discusses each of these topics (lying, affiliation and altruism, compliance, and social reciprocity) as potential explanatory concepts for false alibi corroboration behaviour.

Altruistic lying. A lie is a social interaction in which someone deliberately attempts to deceive someone else. Lying is very common. Social scientists who have conducted diary studies report that people lie on average one to two times per day (DePaulo, Kashy, Kirkendol, Wyer, \& Epstein, 1996; Serota, Levine, \& Boster, 2010). 
However, the distribution of lies told tends to be very skewed, with few people telling most of the lies. For example, in a study by Serota et al. (2010, study 1) in which a representative sample of community members was asked to report the lies they told in the previous 24 hours, the mode and the median were both zero. Only $40 \%$ of the sample reported having lied in the previous 24 hours, but of those respondents the average number of lies told was just over four, and $23 \%$ of all lies were told by only $1 \%$ of the sample. Nevertheless, almost everyone lies: estimates of the proportion of people who tell at least one lie in any given week sits at around 92\% (Serota et al., 2010).

There is some evidence to suggest that as individuals age, from adolescence to late adulthood, they tend to lie less, and they find lying less acceptable (Serota et al., 2010). Further support for this idea can be found in studies that compare college and community samples, which report a higher average frequency of lying for the former than the latter (e.g., DePaulo et al., 1996). Both young and old, however, tend to tell lies that have similar content (i.e., the subject matter of their lies, for example lies about how one feels) and for the same reasons (i.e., motivations, such as to benefit the self or someone else, DePaulo et al., 1996). The large majority of lies are told for selfish reasons (Camden, Motley, \& Wilson, 1984). Overall, people lie more often to strangers than they do to close friends or partners (Ennis, Vrij, \& Chance, 2008).

Several different types of lies have been identified and different classification systems have been proposed based on the liar's motivation, the beneficiary of the lie, the cause of the lie (i.e., the precipitating event), the content of the lie, and/or the relationship between the lie-teller and the target of the lie (e.g., Camden et al., 1984; DePaulo, Ansfield, Kirkendol, \& Boden, 2004; Kashy \& DePaulo, 1996; Ennis et al., 2008). Of 
particular interest for this discussion is the classification based on the beneficiary of the lies. 'Self-oriented' lies are those that benefit the liar, whereas 'other-oriented' lies benefit another person, such as the other person involved in the interaction. When an alibi witness lies by knowingly corroborating a false alibi, the lie is told not to benefit the person being lied to (i.e., the police, lawyer, or jury), but to benefit a third party (i.e., the suspect or defendant). ${ }^{2}$ Thus, false alibi corroboration is a kind of 'other-oriented' lie, but should be further sub-classified as an 'altruistic lie'. This would leave all other 'other-oriented' lies as those that benefit the person to whom the lie is being told.

Although most studies do differentiate between self-oriented lies and otheroriented lies, very few studies make the distinction between other-oriented lies that are meant to benefit the person to whom the lie is being told and those that are meant to benefit a third party (i.e., altruistic lies). Most studies thus include altruistic lies in their 'other-oriented' category (although see Camden et al., 1984; Ennis et al., 2008). The frequency with which these two types of lies are told, however, is not equal: Camden et al. (1984) reported that, of the other-oriented lies told by their participants, only $10 \%$ were altruistic, with the remaining lies benefitting the person to whom the lie was told. It is therefore difficult to generalize some of the findings from the literature on lying behaviour to altruistic lying situations, such as when an alibi witness corroborates a suspect's false alibi. For example, DePaulo and Kashi (1998) found that other-oriented lies are more likely to be told to close friends than to strangers. However, it is likely that most of these lies were told to benefit the friend in question (e.g., to avoid hurting her

\footnotetext{
${ }^{2}$ This is assuming, of course, that the alibi witness has not been threatened or blackmailed by the alibi provider, in which case there would be a benefit to the liar and the lie could be classified as a 'self-oriented' lie.
} 
feelings by hiding one's true feelings about her new clothes) as opposed to a third person, and it is questionable whether this finding would hold for altruistic lies (i.e., that altruistic lies are more likely to be told to close friends than to strangers). In another study by Kashy \& DePaulo (1996), participants who had lower-quality same-sex relationships were consistently found to tell the most lies (both self-oriented and other-oriented, although this relationship has not been further explained or explored since). However, it is possible that individuals who are most likely to tell the most altruistic lies are those who have very good relationships, at least with the beneficiaries of the lies. In fact, one commonly reported reason for lying is to manage relationships (e.g., Camden et al., 1984; DePaulo et al., 1996; Ennis et al., 2008; Kashy \& DePaulo, 1996; Vrij, 2008). For example, someone might lie to a friend about her positive feelings toward the friend's new engagement because telling the truth about her dislike for the fiancé would place their friendship in jeopardy. Similarly, a person lying to the police, an attorney, or a judge about a suspect or defendant's whereabouts may be doing so for very similar reasons: to maintain a good relationship with their friend.

Nevertheless, it may be possible for some of the findings from the literature on other-oriented lies to apply to altruistic lies. For example, several studies found that college-age women are more likely than men to tell other-oriented lies (e.g., DePaulo et al., 1996; Vrij, 2008). In DePaulo et al.'s (1996) 7-day diary study, 15\% of men's lies were other-oriented, whereas $32 \%$ of women's lies were other-oriented. This trend was especially pronounced in same-sex interactions. In other words, women were more likely to tell an other-oriented lie to another woman than men were to tell an other-oriented lie to another man. Also, all participants reported telling more other-oriented lies to women 
than to men. If we assume, as is suggested by Camden et al. (1984), that most of these other-oriented lies are of the type told to benefit the person being lied to, we can conclude that people are more likely to tell lies that benefit women than lies that benefit men. This gender discrepancy is perhaps due to traditional gender roles that place women as the emotionally sensitive and helpless gender (e.g., Diekman \& Eagly, 2000), and is also in line with the finding from a meta-analysis on helping behaviour that women are the recipient of help, from either gender, more often than are men (e.g., Eagly \& Crowley, 1986).

In a study in which altruistic lies were differentiated from other-oriented lies, Ennis et al. (2008) compared the frequency with which individuals told altruistic lies for the benefit of a close friend or romantic partner with the frequency with which they told altruistic lies for the benefit of strangers. Based on answers to survey questions, they concluded that people told altruistic lies benefitting a close friend more often than altruistic lies benefitting strangers (although they did not tell more altruistic lies benefitting a romantic partner than benefitting strangers). It is possible, however, that Ennis et al.'s (2008) findings may simply be due to their participants having more opportunities to lie about their close friend than opportunities to lie about strangers, which would tell us nothing about their general willingness to lie about a close other compared to a stranger. Therefore, it is still unclear whether the rate of altruistic lying is dependent on the closeness of the relationship between the liar and the beneficiary of the lie (as most of the literature on alibi believability would suggest).

Finally, it is important to note that the existing literature on lying deals almost exclusively with everyday “white lies” (e.g., DePaulo et al., 1996; Serota et al, 2010, 
although see DePaulo et al., 2004) which may significantly differ in their frequency, qualities, and predictors from more serious lies. In an alibi corroboration context, a witness is lying to a judge or police officer in order to protect another person. In DePaulo et al,'s (2004) study of serious lies, only 1.3\% of their participants' most serious lie ever told could be classified as having an altruistic motive (i.e., to protect another person) and a bad behaviour as a precipitating event (i.e., originated from wanting to cover up a bad behaviour). Thus, it would appear that serious lies told specifically to cover up another person's bad behaviour are quite rare, perhaps due to a lack of opportunity to do so. However, it is also possible that lies of the kind told when an alibi witness knowingly corroborates a false alibi are not adequately captured or classified even in research that deals with serious lies. This speculation comes from the finding in Marion and Burke (2013) that many alibi witnesses may lie not to cover up a bad behaviour, since many did not think the alibi provider was guilty of one, but instead to divert blame from a suspect whom they believed was innocent. Because of their certainty that a suspect is innocent, some alibi witnesses may not perceive a false corroboration as a serious lie, or even a lie at all.

In short, there is a lack of research on lying that is directly applicable to situations in which an alibi witness knowingly lies for a suspect or defendant. The research on altruistic lying that does exist offers very little empirical evidence to support the common sense notion that lies told to protect a third person are more likely to protect someone who is close to the liar than to protect someone distantly related or unknown to the liar (Ennis et al., 2008). Still, research and theory on altruistic behaviour (other than lying) would still suggest that this might be true. 


\section{Affiliation and altruism.}

Family affiliation. Evolutionary theories of kin selection and reciprocal altruism both offer an explanation as to why someone who is closely related to a suspect or defendant would be more likely to help him or her by corroborating a false alibi than someone who is unrelated or distantly related. Hamilton's (1964) theory of kin selection states that an individual (whether human or an another animal) is likely to act altruistically towards others if the survival advantage of his or her genetic material gained by the altruistic act outweighs the personal costs, or risk, associated with the act. In other words, the result of helping (or not) will depend on the degree of genetic relatedness between the two individuals, the degree to which the altruistic act will increase the chances that the genes of the person being helped will survive and remain in the gene pool, and the level of risk to the helper's genes' ability to remain in the gene pool. Thus, when the risk to the helper and the impact of the altruistic act are kept constant, an individual is more likely to help a person with whom he or she shares more of his or her genetic material than someone with whom he or she shares less genetic material. Kin selection theory would therefore predict an evolutionary advantage for the genes of alibi witnesses who lie to protect suspects or defendants who are biologically related to them, such as a child, sibling, or nephew. Of course, there is a risk involved with corroborating a false alibi (e.g., charges of obstruction of justice, perjury, or even imprisonment, if caught). Also, the impact of corroborating a false alibi on the survival advantage of the alibi corroborator's genes depends on several factors, for example the age and reproductive ability of the alibi provider. However for any one situation, when all factors are fixed, kin selection theory would predict that the closer a witness is to an alibi 
provider, biologically-speaking, the more likely it is that this witness will corroborate an alibi that is false. This idea fits nicely with Hosch et al.'s (2011) findings described in the alibi literature review above.

The principle underlying Hamilton's (1964) kin selection theory can be used to explain many day-to-day human behaviours. For example, it can explain why people tend to leave most of their belongings and wealth to close relatives than distant or nonrelatives in their will, and why people tend to buy more and bigger gifts for immediate family members (e.g., during Christmas) than to nieces and cousins. The theory has also been supported by experimental and correlational research. For example, Madsen et al. (2007) had participants in both Western and non-Western cultures hold a painful isometric physical exercise for as long as they could, and informed them that the duration achieved would determine how much of a highly-valued commodity (money for Western participants and food for non-Western participants) would be received by recipients whose genetic material overlapped to different degrees with the participant (e.g., 100\%, $50 \%, 25 \%, 12.5 \%$, and $0 \%$ ). Participants held the position for longer (i.e., suffered higher 'cost') when the commodity (for which its gain was used as a proxy for increased gene survival) was given to recipients who shared more of their genes with the participant than when it was given to recipients who shared fewer. The linear trend was significant for both men and women, in both cultures, and remained significant even when the recipients' past and future help toward participants (i.e., perceptions and expectations of reciprocal help) was controlled for. In another study, David and Daly (1997) conducted a secondary analysis of Statistics Canada data on the childcare practices of over 3000 Canadians, and found that adults were more likely to provide childcare for family 
members than for friends, but only when they did not already have young children living with them (and thus could not expect the recipient of the help to reciprocate the childcare). This finding, they suggested, supports the notion that true altruistic behaviour (i.e., help that is not expected to generate help or compensation in return) is more likely to occur between relatives than between non-relatives (David \& Daly, 1997).

Social affiliation. Of course, not all altruistic behaviours are directed at relatives. For example, according to Hosch et al. (2011), many of us say we would be willing to lie in court to protect our best friend, or even a co-worker. Trivers' (1971) reciprocal altruism theory can help explain why we act altruistically towards individuals who are socially - as opposed to biologically - related to us, and how this can be adaptive from an evolutionary point of view. It states that helping a person in need, even if there is a cost associated with this act, is beneficial to the helper if, in the future, this person is likely to reciprocate by helping in return. Thus, altruism is most likely to exist between individuals who are part of the same community or belong to common groups (i.e., are socially related) and who therefore are likely to see each other and interact again in the future. The theory works best among members of small, tight-knit communities, as one member who acts altruistically toward another is likely to be around the same individual when he or she is in need of help in the future.

It is not difficult to find examples of reciprocal altruism in the day-to-day lives of humans, but in addition to anecdotal evidence, empirical evidence also supports a relationship between altruism and reciprocity. In a self-report questionnaire study, Stewart-Williams (2007) found that how much help is given to a particular person (e.g., emotional support, help during a crisis, household help) is a significant predictor of how 
much help is received by that same person. This was true for both relatives and nonrelatives, but the trend was especially pronounced for acquaintances. This suggests that people may be motivated to help non-related individuals because they have a reasonable expectation that the help will be reciprocated in the future. In fact, Davis and Daly's (1997) analyses of Canadian childcare practices demonstrated that adults who did have young children living at home were more likely to provide childcare for friends than for relatives, presumably because it was expected that these friends would reciprocate the childcare support (whereas this expectation may not exist between family members). The theory of reciprocal altruism can therefore explain why non-relatives such as friends, coworkers, and neighbours may lie for one another by corroborating a false alibi despite their lack of biological relationship, and why close friends and individuals who share memberships to several small social groups and interact often may be more likely to do so than individuals who have a single common membership and interact infrequently.

Liking and compliance. Sharing a close biological or social relationship with another could lead to altruistic acts, even in the absence of a direct request to help from the recipient. Thus, even without being directly asked to lie by her son, a mother might choose to lie to police by telling them that her son was at home with her the previous evening when she realizes the potential costs to her son's wellbeing if she tells the truth. However another potential scenario is that a criminal suspect who gave police a false alibi may ask someone else to lie for him or her by corroborating the false alibi. How close this person is to the suspect (biologically or socially) is likely to influence whether or not he or she complies with the direct request, but compliance is also affected by other factors. For example, individuals are more likely to comply with requests from 
individuals they like than from those they do not like (Cialdini \& Trost, 1998). Since it is reasonable to assume that we like our friends and family members more than we like strangers, it would then follow that we should be more likely to corroborate a false alibi for these friends and family members. Nevertheless, all else being equal, a suspect who is liked by a potential alibi corroborator should have a better chance of having a false alibi corroborated by him or her than a suspect who is not liked.

The degree to which two individuals like one another can and has been experimentally manipulated. Social psychologists have increased liking between participants by increasing their perceived similarity in personality characteristics, opinions, or even first names (Byrne, 1997), by increasing the time spent interacting with one another (e.g., Insko \& Wilson, 1977), or by increasing the degree to which one person believes another likes them in return (e.g., Berscheid \& Walster, 1978).

Experimental manipulations of liking and perceived similarity between individuals have also been shown to lead to an increase in compliance (e.g., Burger et al., 2001; Burger, Messian, Patel, del Prado, \& Anderson, 2004). For example, in a series of studies by Burger et al. (2001), participants who had either previously interacted with the confederate, believed that they had similar personality traits as the confederate, or had spent time in close physical proximity to the confederate, were more likely to comply with a confederate's request to read and provide feedback on an eight-page essay than participants in respective control conditions. The effect they found was partially moderated by how much the participants liked the confederate, thus reinforcing the idea that liking increases compliance. In another study, Burger et al. (2004) manipulated characteristics of the confederate that should be unrelated to how likeable he or she was, 
but that were either similar to the participant, or not (birth date, fingerprint type, and first name). Participants who thought they shared a birthday or a rare fingerprint type with a confederate were more likely to comply with the same essay feedback request as in Burger et al. (2001) than when they thought they had a different birthday or fingerprint type, and participants donated more money to a charity when they thought the requestor had the same first name as them than when the requestor had a different name. It seemed, though, that when the participants thought they had something in common with a confederate that was very common in the general population (e.g., a common fingerprint type), the perceived similarity no longer increased compliance, nor liking of the confederate (Burger et al., 2004). Thus, sharing even arbitrary personal characteristics with someone, when these are relatively rare, can lead to an increase in compliance rates, presumably through an increase in liking of, or feelings of affiliation with, the person making the request.

Burger and colleagues $(2001 ; 2004)$ suggest that when people respond to a request, at least in face-to-face situations, they do not do so based on thoughtful considerations of the requests. Instead, they claim, people use a cognitively economical social heuristic that would lead them to comply with requests from those who are likeable and those who are similar to them. This would serve the adaptive goal of quickly and appropriately responding to friends and family members (i.e., those in our environment who we should be able to trust). The increased liking between the participant and the confederate demonstrated in the above studies, however ephemeral, would have activated this heuristic and promoted compliance (Burger et al., 2001; 2004). Following this logic, it is therefore possible that if an individual is approached by a friend or family member 
(or anyone else likeable and similar to them) and asked to corroborate his or her false alibi, the automatic and socially-appropriate response would be to comply with the request and lie on the suspect's behalf.

Reciprocity and social responsibility. Sometimes altruistic behaviour can't be described in terms of complying with a request or an innate drive to pass on our genetic material. At times people help others simply because they feel it is their social responsibility to do so. According to the reciprocity norm, individuals are motivated to help those particular individuals who have helped them in the past (Gouldner, 1960). (In contrast to Trivers' theory of reciprocal altruism, the reciprocity norm states that we help others in order to repay them, as opposed to helping others in order to receive future help.) The social responsibility norm, on the other hand, states that individuals do good to others in response to good that has been done to them in the past and will be done in the future, without any specific expectation of reward from a particular person at a particular time (Berkowitz, 1972).

Berkowitz and Daniels (1963; see also Daniels \& Berkowitz, 1964) have argued that the social responsibility norm should be most salient when the person receiving help is dependent on the person providing the help necessary for achieving a particular goal, and that the reciprocity norm should be most salient when the person expected to provide help has received help in the past. Several studies (Berkowitz \& Daniels, 1964; Daniels \& Berkowitz, 1963; Goranson \& Berkowitz, 1966) provide support for both norms and also demonstrate how dependency and prior help can activate different norms to affect helping behaviour. In these studies, a participant and confederate complete two tasks. In Berkowitz and Daniels' (1964) study, prior help as well as dependency of the confederate 
on the participant was manipulated. In the first task, the confederate either did or did not help the participant with her task, and in the second task the experimenter's evaluation of the confederate was either dependent or not dependent on the participant's productivity. Because the second task was easy and mundane (i.e., assembling paper boxes), the participants' productivity was taken as a measure of their effort in the task. Participants' productivity was significantly higher when the confederate's evaluation depended on them than when it did not, regardless of whether the confederate had previously helped the participant or not (i.e., even when there was nothing in it for the participant), suggesting the social responsibility norm was at play. However, dependency motivated participants even more when they had previously received help from the confederate than when they had not. In other words, prior help and dependency seemed to have a cumulative effect on participants' motivation to help their study partner obtain a good evaluation. The same results were replicated when dependency and liking (of the confederate) were manipulated (Daniels \& Berkowitz, 1963), again suggesting that liking and dependency also seemed to have a cumulative effect on participants' motivation to help.

Findings from other studies suggest that in a situation in which someone receives prior help, the reciprocity norm is more salient than the social responsibility norm. In a third study employing a similar experimental paradigm as Berkowitz and Daniels (1964), Goranson and Berkowitz (1966) manipulated whether the confederate whose evaluation depended on participants in the second task was the same or a different person as the confederate who either helped them or refused to help them in the first task. This time, prior help increased participants' performance only when they were working with the 
same confederate for both tasks. In other words, participants exerted an increased amount of effort to help the person who helped them in the first place, whereas the amount of help given to a new person was consistent regardless whether or not they had been previously helped. Regan (1971) further investigated the effects of a previous favour on subsequent compliance with a request. He manipulated whether a participant received a favour (was bought a soft drink) from a confederate, from the experimenter, or from neither, and measured how many charity raffle tickets participants subsequently bought from the confederate. Those who received a soft drink from the confederate bought significantly more tickets than those who received a soft drink from the experimenter or those who did not receive one. Thus, having received a favour did not seem to increase one's motivation to help others in general. In both studies, individuals seemed especially motivated to help others who had previously helped them.

Results from the above studies suggest that, in an alibi corroboration context, an alibi witness may be particularly likely to lie when he or she feels that the defendant is dependent on his or her testimony and that corroborating the false alibi may be the only way to protect the defendant's freedom, or when the alibi witness feels the need to reciprocate help that the defendant may have provided in the past. In addition, this increased propensity to lie may be particularly likely if the alibi witness feels a certain affinity for the defendant.

A single published study investigating the effect of prior help on altruistic lying was found. In this study the participants were children and the study was designed from a developmental perspective (Greenglass, 1972). The experimental paradigm involved 8and 12-year-old boys drawing a picture that was judged either favourably or poorly by a 
confederate of the same age. The children were told that they could win a prize if their drawing was rated favourably, thus participants were 'helped' if the confederate had given them favourable ratings. While the experimenter was away, this same confederate accidentally broke a vase and asked the participant not to tell the experimenter. When questioned about how the vase broke, the proportion of boys who lied to protect the confederate was greatest in the group of 12-year-olds who had previously received help. This might suggest that a certain level of cognitive development is necessary to recognize and act upon the social responsibility and reciprocity norms, and that perhaps all else being equal, an alibi witness who is a young child may not behave the same (i.e., may be less likely to lie) as older children and adult witnesses (as has been suggested by Dahl \& Price, 2012). Thus, because the different cognitive and moral developmental stages of children and adults may impact lying behaviour (see also Fu, Evans, Wang, \& Lee, 2008, for example), the focus of the current research will remain on adult alibi witness behaviour.

Summary. We can conclude from this brief review of the literature on helping, lying, and altruism that certain situational and personal characteristics can either increase or decrease the likelihood that a potential witness would lie by corroborating a false alibi. Some evidence from the lying literature suggests that, in general, older individuals may be less likely to lie (e.g., Serota et al., 2010), and that women may be more likely than men to be the beneficiaries of lies, especially when lies are told by women (DePaulo et al., 1996). There is also some evidence that altruistic lies are told more often to protect closely related others than to protect strangers, although this finding has not been consistent and needs further evidence to support it (Ennis et al., 2008). Evolutionary 
theories of altruism, however, would predict that individuals who are closely related, either biologically or socially, to a suspect or defendant may gain a survival advantage by helping the latter keep his or her freedom (Hamilton, 1964; Trivers, 1971) and thus should be more likely to corroborate a false alibi than more distantly related individuals. Alibi providers who are generally likeable and who find themselves having commonalities with a potential alibi witness may be more likely to have their false alibis corroborated than suspects who are not particularly liked and who do not share similarities with potential corroborators (e.g., Burger et al., 2001; 2004). Finally, a potential alibi witness who has been helped by a suspect or defendant in the past, and who feels that the alibi provider's freedom and well-being is dependent on them may be more likely to lie than those potential witnesses who have never received help from the suspect and do not feel responsible for helping (Berkowitz \& Daniels, 1963).

Of course, a witness who knowingly lies by corroborating a suspect's false alibi can be perceived as being helpful toward the suspect just as much as a witness who tells the police, attorney, or judge the truth can be perceived as being helpful to the criminal justice system. Thus, the same situational and personal factors that impact the likelihood that a witness will lie for a suspect may impact the likelihood that the witness will tell the truth to the police, an attorney, or a judge (e.g., how much the witness likes a police officer, or how much the two have in common). The studies described herein, however, focus on help and altruism between an alibi witness and a suspect. 


\section{Overview of the current studies}

Findings from the literatures on alibi evidence as well as from social and evolutionary psychology theory would support the expectation that close others are motivated to lie to protect one another and are therefore less believable as witnesses than unrelated others. Some of the most directly relevant supportive findings, however, came from self-report measures only (e.g., Hosch et al., 2011). As humans we often think and say we would behave in a particular manner in a given hypothetical situation (e.g., rushing to help an injured person on a busy street), but behavioural data often prove us wrong (e.g., Darley \& Latané, 1968).

The goal of the proposed research is to identity factors that, based on evolutionary and social psychology theories, are likely to impact the probability that a witness will lie for a suspect in an alibi corroboration context. Three studies will follow the basic experimental paradigm developed and used by Marion and Burke (2013) described earlier, with some modifications necessary to test the variables of interest.

In most previous studies on liking and compliance, and the few on altruistic lying, the individual needing help made an explicit request to participants. In Marion and Burke (2013), however, the confederate implicitly requested the participant's help by lying to the experimenter in the presence of the participant. It is possible that making a request to lie more explicit may increase participants' perceived responsibility to 'help', thereby increasing the rate of corroboration, especially between individuals who like one another. Thus, study 1 investigated whether a direct request from the confederate to not reveal his or her brief absence during the study would increase the likelihood that participants would lie by corroborating the confederate's false alibi (that the latter was in 
the study room during the entire study session). Study 1 also served as a replication of Marion and Burke's (2013) liking manipulation. Our lack of a significant effect of liking on the rate of false alibi corroboration came as a surprise, given the current standing of the alibi evidence literature and the established evolutionary psychology and social psychology theories on altruism.

Study 2 investigated whether the norm of reciprocity could induce participants to lie for a confederate by corroborating his or her false alibi. In this version of the experiment, the confederate sometimes helped the participant complete a difficult task before he or she was asked to corroborate the false alibi. The corroboration rate of participants who were previously helped was then compared to the corroboration rate of those who were not. Also, because men tend to help women more than other men, and women help other women and men equally (and thus women receive more help than do men, including help in the form of lying for their benefit, Camden et al., 1984; Eagly \& Crowley, 1986), study 2 compared the corroboration rates of men and women.

Finally, in study 3 , a more externally valid test of the effect of relationship was achieved by recruiting pairs of actual friends and comparing their rate of false corroboration to that of pairs of strangers. In this study, one participant from each pair was recruited to act as the 'confederate' who was suspected of stealing money. This permitted a more direct test of whether the relationship between two individuals is likely to influence altruistic lying (as opposed to using liking as a proxy for friendship). 


\section{Hypotheses.}

Hypothesis 1. It was predicted that participants who underwent liking-enhancing activities with a confederate would be more likely to corroborate the confederate's false alibi than participants who did not go through the liking-enhancing manipulation (Study 1).

Hypothesis 2. It was predicted that participants who were directly asked not to reveal the confederate's whereabouts would be more likely to corroborate the confederate's false alibi than participants who did not receive such a request (Study 1).

Hypothesis 3. It was predicted that participants who were previously helped by a confederate would be more likely to corroborate the confederate's false alibi than those who were not previously helped by the confederate (Study 2).

Hypothesis 4. It was predicted that the proportion of women who corroborated the (female) confederate's alibi would be higher than the proportion of men who corroborated the (male) confederate's alibi (Study 2).

Hypothesis 5. Finally, it was predicted that participants would be more likely to corroborate a friend's false alibi than a stranger's false alibi (Study 3). 


\section{Study 1 - Effect of liking and social pressure on corroboration of a false alibi}

Under the pretext of a problem-solving study investigating the role of personality similarity on performance, participants interacted with a confederate. During the first part of the experimental session, the participant's liking of the confederate was manipulated. In the 'friendship-enhancing' condition the participant-confederate pair was told that their personalities were very similar (based on a personality measure they had completed at the beginning of the term), and they then completed a collaborative task. In the 'stranger-maintaining' condition, the pairs were told that they had very different personalities, and they then completed individual tasks. During the second part of the experimental session, all participant-confederate pairs completed a collaborative task. During this task the confederate momentarily left the study room under pretext of needing to use the restroom: In the 'request' condition, the confederate returned to the study room and asked the participant not to reveal his or her absence to the experimenter. In the 'no request' condition, the confederate made no such request. The pair was then questioned, together, about a mock theft that had just occurred nearby, for which the confederate offered a false alibi. The participant was later questioned individually and given the opportunity to either corroborate the confederate's alibi that he or she remained in the study testing room during the entire study task, or to refute it. The design of this study was a 2 (relationship condition) $\times 2$ (request condition) between-subjects factorial. The main dependent variable was the participant's corroboration or non-corroboration of the confederate's alibi. 


\section{Participants.}

Ninety-three participants took part in the study. All participants were undergraduate students enrolled in an introductory psychology course and each received one course credit as compensation for their participation. Their age ranged from 17 to 38 years with a median age of 18 years. The study was advertised on SONA, an online experiment management system, under the title "Does Personality Affect Group Performance?" (see all study materials for study 1 in Appendix A). Males and females were recruited separately via two SONA studies, one advertising for male participants only and the other for female participants only, to ensure that the confederate was always of the same gender. Only students who had previously completed a pre-screen survey on the SONA system (which included a personality questionnaire - see Measures section) were able to sign up for this study. Although the results from the pre-screen questionnaire were not of interest in this study, it was necessary for participants to complete it because the cover story for the study was based on this personality measure.

Seven participants were dropped from the data set and subsequent analyses due to their strong disbelief of the cover story, as became evident during the debriefing session. Three more participants were excluded because they did not hear or understand the mock theft scenario, an additional two were excluded because they did not hear the confederate's alibi (due to the participant speaking over the alibi), and a final participant was excluded due to having already participated in the study the previous term (but only advised the experimenter during debriefing). Thus, the data from a final sample of 80 participants (58 females) were retained for analyses. 


\section{Setting and Apparatus}

The experimental session took place in a testing room adjacent to the lab office. At the start of the session and during the group tasks, the participant and confederate were seated opposite one another at a table. The experimenter was not in the room during the tasks, and the door was kept shut at all times, except for when the experimenter entered the room to provide materials and instructions.

The stimuli for some of the group tasks were presented on a laptop computer, and all other tasks and questionnaires were completed via paper and pen. A bogus heart rate monitor (made of scrap electrical pieces - see Figure 1) was used during the second part of the session as part of a task assigned to the participant (described later). This monitor was connected to the laptop computer via a USB cable, and a "sensor pad" at the end of a connected wire was affixed to the participant's finger.

A Sony ICD-PX720 digital audio recorder was used to record the participant's rejection or corroboration of the false alibi at the end of the session.

\section{Measures}

Pre-screening personality questionnaire. All participants answered the TenItem Personality Inventory (TIPI, Gosling, Rentfrom, \& Swann, 2003) as part of a larger online questionnaire completed at the beginning of the academic term (SONA prescreen). The inventory was used to increase the believability of the cover story, but personality characteristics were not of interest in this study and thus scores were not recorded and will not be reported. 


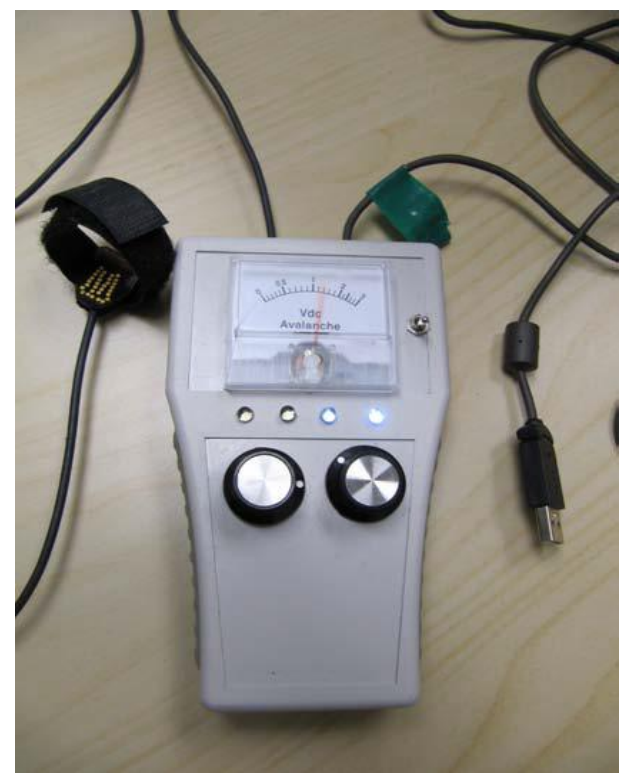

Figure 1. Bogus heart-rate monitor

Liking questionnaire. To ensure that the liking manipulation was successful, participants were presented with a questionnaire containing ten Likert-type statements assessing how much the participant liked the confederate and how willing they would be to pursue a friendship with him or her (e.g., Would you enjoy time spent with this person?). This measure has previously been shown to be highly reliable (ten items; $\alpha=$ .93; Marion \& Burke, 2013). The Liking questionnaire items were embedded amongst other demographic questions (gender, age) and filler questions relating to the wordgeneration task.

Post-measure questionnaire. Participants answered this questionnaire at the end of the experimental session. It consisted of only filler questions related to the last collaborative task, and was administered in order to provide an opportunity for the participant and confederate to be separated and for the experimenter to question the participant about the confederate's alibi (see Procedure section). 
Debriefing questionnaire. Participants were asked a series of questions during the debriefing interview in order to assess the efficacy of the manipulations. The experimenter asked participants whether they heard the confederate provide the false alibi, whether the confederate had asked them not to report that he or she went to the restroom, to what extent they suspected that the confederate was guilty of stealing the money (on a 10-point Likert scale), and how much they liked the confederate at the end of the study (on a 7-point Likert scale). Participants were also asked to explain why they did or did not corroborate the confederate's alibi.

\section{Procedure}

Participants were randomly assigned to one of two relationship conditions: friendship-enhancing (FE) or stranger-maintaining (SM), and to one of two request conditions: request $(\mathrm{R})$ or no-request $(\mathrm{NR})$. The experimenter was blind to the request condition assignment until the end of each session. The confederate, however, had access to the condition assignment list and thus was able to act accordingly for each session.

From the SONA study ad, participants were informed that the purpose of the study was to investigate the influence of personality similarity on peers' performance on problem-solving tasks. Participants were tested in pairs, however the second 'participant' was a confederate. The pair (i.e., the participant and confederate) was scheduled to arrive at the laboratory at the same time. They were presented with consent forms to read and sign, and were told that based on their score on the pre-screen personality questionnaire, they had been assigned to one of two 'personality' conditions (i.e., one of the two relationship conditions). In the FE condition the participant and confederate were told that they had similar scores on the personality measure and thus had been assigned to the 
'similar personalities' condition. In the SM condition they were told that they had very different scores on the personality measure and thus had been assigned to the 'different personalities' condition. The pair was then asked to complete another filler personality questionnaire, under the pretext of needing a second personality measure to ensure that the pre-screen personality scoring was accurate and that they had indeed been assigned to the correct 'personality' condition. All pairs were told that their score on this measure confirmed their group assignment. It was anticipated that a participant who perceived the confederate to be similar to him or herself would feel an increase in liking toward the confederate as compared to a participant who perceived the confederate to be dissimilar (e.g., Byrne, 1971; 1997; Daniels \& Berkowitz, 1963).

Verbal instructions for the tasks to be completed for the study were then given to the participant and confederate. Only the instructions for the first task differed between the FE and SM conditions. In the FE condition, the first task was done collaboratively to allow for the friendship-enhancing manipulation, whereas in the SM conditions it was done individually, in separate rooms, to limit the interaction and potential increase in liking between the participant and confederate. The experimenter provided the necessary material at the start of each task and questionnaire, but was not present during their completion.

Word generation task. In the FE condition the first task consisted of an 8-minute collaborative word-generation filler task, for which the participant and confederate were instructed to work together to find words in a large letter grid. In the SM condition, the confederate and participant were separated and the participant completed the same 8minute word generation task alone. The interaction necessary to complete the task 
collaboratively was expected to increase liking between the participant and the confederate in the FE condition. In addition, during this task (in the FE condition only), the confederate pretended to be in the same psychology class as the participant and invited him or her to a study group for an upcoming exam. This was done in order to create the expectation that the participant would likely see and interact with the confederate again in the future. This is important as we assume that in most cases in which a friend of a suspect is asked to corroborate the suspect's alibi, this friend expects to see and interact with him or her in the future, and this fact may impact their decision to either corroborate or reject a false alibi.

Liking questionnaire. After the word generation task the participants in both conditions completed the Liking questionnaire. Participants in the FE condition who were with the confederate during the word generation task were separated for the completion of this questionnaire, and participants in the SM condition remained alone.

Restroom break. The second 'problem-solving' task was a collaborative one for pairs in both conditions. The experimenter instructed the participant and confederate that she had to leave briefly to pick up office supplies in the next-door building, but that she would return before the end of their tasks. This was done in an attempt to ensure that the participant believed that the experimenter would not be watching them or monitoring their actions them during this the task. Before leaving, the experimenter provided the instructions for three filler tasks: The first part consisted of an anagram activity. The second part was an image-rating activity, for which the participant rated the attractiveness of abstract images. The third part was a memory activity. The experimenter explained that the goal of the image-rating activity was to obtain pre-ratings for an upcoming study, 
and that it would require a physiological measure (heart rate). The experimenter then informed them that since there was only one heart rate monitor, only one person would be able to complete the activity. The participant was told that he or she was randomly selected to do the image ratings, and that for reasons of convenience the physiological measure would be recorded throughout the entire duration of the three tasks. This was done in order to later exclude the participant as a suspect in the upcoming mock theft. The participant's hand was strapped to the bogus heart-rate monitor ${ }^{3}$ and the apparatus turned on. After providing all instructions and material for the three tasks, the experimenter left the room. It was during the image-rating task that the confederate left the room, under pretext of needing to use the restroom while his or her participation was not needed. The confederate returned to the room three minutes later. In the request condition he or she asked the participant to not reveal his or her absence to the experimenter by saying: 'I'm not sure if I was allowed to leave, so don't tell her I left, ok?" In the NR condition the confederate returned to the room and continued the tasks without making any such request.

Mock theft and false alibi. Nearing the end of the memory activity, the experimenter momentarily opened the door to the study room to announce her return. Upon completion of the task, the experimenter re-entered the room (with a concealed audio-recorder) and asked the pair, in a concerned tone, if they had seen or heard anyone enter the adjacent room during her absence, as some money was stolen. Immediately after the experimenter's question, the confederate denied any involvement and stated that both

\footnotetext{
3 The monitor was connected to a nearby computer and participants were told that a computer program was continuously recording their heart rate. The participants' heart rates, however, were not actually recorded.
} 
he or she and the participant were in the room for the entire duration of the task. The experimenter then sighed, yielded to the denial, and then separated the pair by bringing the confederate to a different room for the completion of the next questionnaire.

Alibi corroboration or rejection. Participants were given a questionnaire with filler questions about the three tasks just completed. During this time, the experimenter approached the participant, turned off and removed the bogus monitor, and told him or her that since a continuous heart rate measure had been recorded during the previous tasks it was evident that he or she did not leave the room, but asked if the confederate also remained in the room for the entire duration of the task. The participant's response was captured by the concealed audio-recorder still on the experimenter. The study concluded after this last questionnaire. The initial instructions given to the participant included a final collaborative task, but no such task took place - it was mentioned only to create an expectation that the participant would have to interact with the confederate again in the near future following the alibi-corroboration question. This was done in another attempt to better mimic actual cases in which individuals are given an opportunity to corroborate an alibi, and expect to see the accused person in the future.

Debriefing interview. The participant was debriefed immediately after the filler questionnaire. Participants were informed of the true identity of the confederate and that no theft had actually occurred, and the experimenter subsequently administered the debriefing questionnaire. Following the debriefing questionnaire, a detailed explanation of the study rationale and research questions was provided, with special attention to the rationale for the use of deception. As an ethical consideration toward participants' wellbeing, the experimenter refrained from using the term "lying", and instead used the 
phrases "cover up for" and "supporting an alibi" when explaining alibi corroboration or describing participants' own corroboration responses. Thus, the altruistic nature of a false alibi corroboration was emphasized. This was done in order to minimize the risk

that participants would experience psychological distress over having been 'caught' in a lie, when they corroborated the alibi. Similarly, when participants did not corroborate the alibi, the honesty and truthfulness of their responses was emphasized in order to minimize the risk that participants would feel bad for 'snitching' on a fellow student. In either case, participants were reassured that their responses were not uncommon. At the end of the debriefing interview a verbal consent for the use of the audio recording was also requested. No participant expressed concerns over the use of deception or the true purpose of the study, and the vast majority stated that they had enjoyed the experience.

\section{Results}

\section{Experimental Manipulation Checks}

Liking manipulation. The Liking questionnaire was used as a measure of how much each participant liked the confederate. Each participant's ratings of the ten items were added to create a single Liking score, with higher scores indicating greater liking. Two participants $(2.5 \%)$ had between one and four missing items, for which person-mean imputations were used (Downey \& King, 1998; Hawthorne \& Elliott, 2005). The measure had excellent internal consistency (Cronbach's $\alpha=.95)$ and reliability, with all corrected item-total correlations ranging from .40 to .86 (Nunnally, 1978).

Liking scores across all participants ranged from 16 to 70 , with a mean of 51.20 (out of a maximum score of $70, S D=13.47$ ). A 2 (relationship condition) $\times 2$ (request condition) ANOVA on participants' Liking score revealed a main effect of relationship 
condition, $F(1,76)=88.35, p<.001, \eta_{p}{ }^{2}=.54$. Participants in the FE condition had significantly higher Liking scores $(M=61.23, S D=6.84, n=39)$ than those in the SM condition $(M=41.66, S D=11.06, n=41)$. There were no differences in Liking scores based on the request condition $\left(p=.81, \eta_{p}^{2}=.001\right)$ and there was no significant interaction $\left(p=.11, \eta_{p}^{2}=.03\right)$. Participants were also asked again, during the debriefing interview, to rate their liking of the confederate (on a 7-point Likert scale). Because participants in both relationship conditions interacted with the confederate during the last half of the study, it was possible that any differences in liking produced by the manipulation at the start of the study would have been obliterated before the critical alibi corroboration phase. A 2 (relationship condition) $\times 2$ (request condition) ANOVA on participants' rating of this single-item liking measure was conducted. Again, a significant effect of relationship condition was obtained, $F(1,74)=13.93, p<.001$, $\eta_{p}{ }^{2}=.16$, in the expected direction (FE condition: $M=6.12, S D=.76$; SM condition: $M=5.38, S D=.96)$. There was no effect of request condition $\left(p=.34, \eta_{p}{ }^{2}=.01\right)$, and no interaction $\left(p=.45, \eta_{p}^{2}=.01\right)$. Thus, the liking manipulation had a strong effect on how much participants liked the confederate, and it can be assumed that this effect lasted throughout the study session.

To check if there were any differences in liking between confederates, a 2 (relationship condition) $\times 4$ (confederate) ANOVA on Liking scores was conducted (although six confederates were used, two of them were involved in only one or two sessions of each of the relationship conditions and were thus excluded from this analysis). As expected, there was again a strong effect of relationship condition, $F(1,67)=55.53$, $p<.001, \eta_{p}{ }^{2}=.45$ (FE condition: $M=60.97, S D=6.92 ;$ SM condition: $M=42.26$, 
$S D=11.09)$. There was also a significant effect of confederate, $F(3,67)=2.97, p=.04$, $\eta_{p}^{2}=.12$. Tukey's HSD post hoc comparisons revealed that one of our female confederates was significantly more liked than one of our male confederates $(p=.004)$ and non-significantly more liked than the other female confederate $(p=.08)$. More importantly, however, there was no interaction between confederate and relationship condition, $p=.52, \eta_{p}{ }^{2}=.03$, suggesting that any differences in confederate likeability was consistent across relationship conditions. In addition, a $t$-test confirmed that this well-liked confederate also obtained significantly higher Liking scores in the FE condition $(M=62.63, S D=6.58)$ than in the $\mathrm{SM}$ condition $(M=45.86, S D=10.54)$, $t(26)<.001, d=1.98$.

Attention to alibi. As was mentioned above, two participants were excluded from the analyses because they did not hear the confederate's alibi. This was explained and confirmed by a replay of the audio recording of the sessions showing that the participant spoke over the confederate's alibi. From this point, confederates were instructed to make eye contact with the participant during the statement of their alibi, and to repeat the alibi if they had any doubt that the participant had heard them (e.g., if they were speaking at the same time). Despite these precautions, a surprisingly high percentage of participants (18.8\%) still reported in the debriefing interview that they did not hear or did not remember hearing the confederate's alibi. In all cases the alibi was heard quite prominently on the audio recordings. It may be that some of these responses reflect self-serving answers, but others may reflect a lack of attention to the alibi. As a precaution, the main analyses were conducted with and without these cases. 


\section{Main Analyses: Liking and Compliance to a Request}

Thirty-four out of 80 participants (42.5\%) corroborated the confederate's false alibi by confirming, when asked by the experimenter, that the confederate had remained in the study room during the time the theft occurred. Of the participants who did not corroborate the alibi (i.e., those who reported that the confederate left to go to the restroom), almost all (43, or 93.5\%) did so when the experimenter asked them to confirm the alibi. The three other participants reported the confederate's absence either by immediately 'reminding' the confederate that they left when the latter stated her alibi, by reporting the absence to the experimenter after the confederate had left but before the experimenter questioned her, or by reporting the absence to the experimenter just before the debriefing started, but after having initially corroborated during the questioning phase. Of the 43 participants who reported the confederate's absence when the experimenter asked for a confirmation of the alibi, 5 participants initially corroborated but almost immediately changed their minds and reported the absence before the experimenter exited the study room.

A three-way hierarchical loglinear analysis was conducted which included relationship condition, request condition, and alibi corroboration outcome. The final model retained the request condition $\times$ alibi corroboration outcome interaction. The likelihood ratio of this model was $\chi^{2}(4)=1.86, p=.76$. As predicted, there was a significant interaction between request condition and alibi corroboration, $\chi^{2}(1)=9.81$, $p=.002$. Of the participants in the request condition, $60.5 \%$ corroborated the false alibi whereas of the participants in the NR condition, $26.2 \%$ corroborated the false alibi. Thus, Hypothesis 2 was supported: The odds ratio revealed that the odds that a participant 
corroborated the false alibi were 4.37 times higher when they were asked not to report the confederate's whereabouts than when they were not asked to do so (Figure 2).

Hypothesis 1, however, was not supported. Participants in the FR condition were not more likely to corroborate the alibi than participants in the SM conditions (35.9\% vs. $48.8 \%$, respectively), $p=.24$. There also was no interaction between relationship condition and request condition on corroboration rates, $p=.96$ (see contingency table, Table 1).

Since several participants reported during debriefing that they had not heard or could not remember the confederate's alibi, and because there was a non-significant

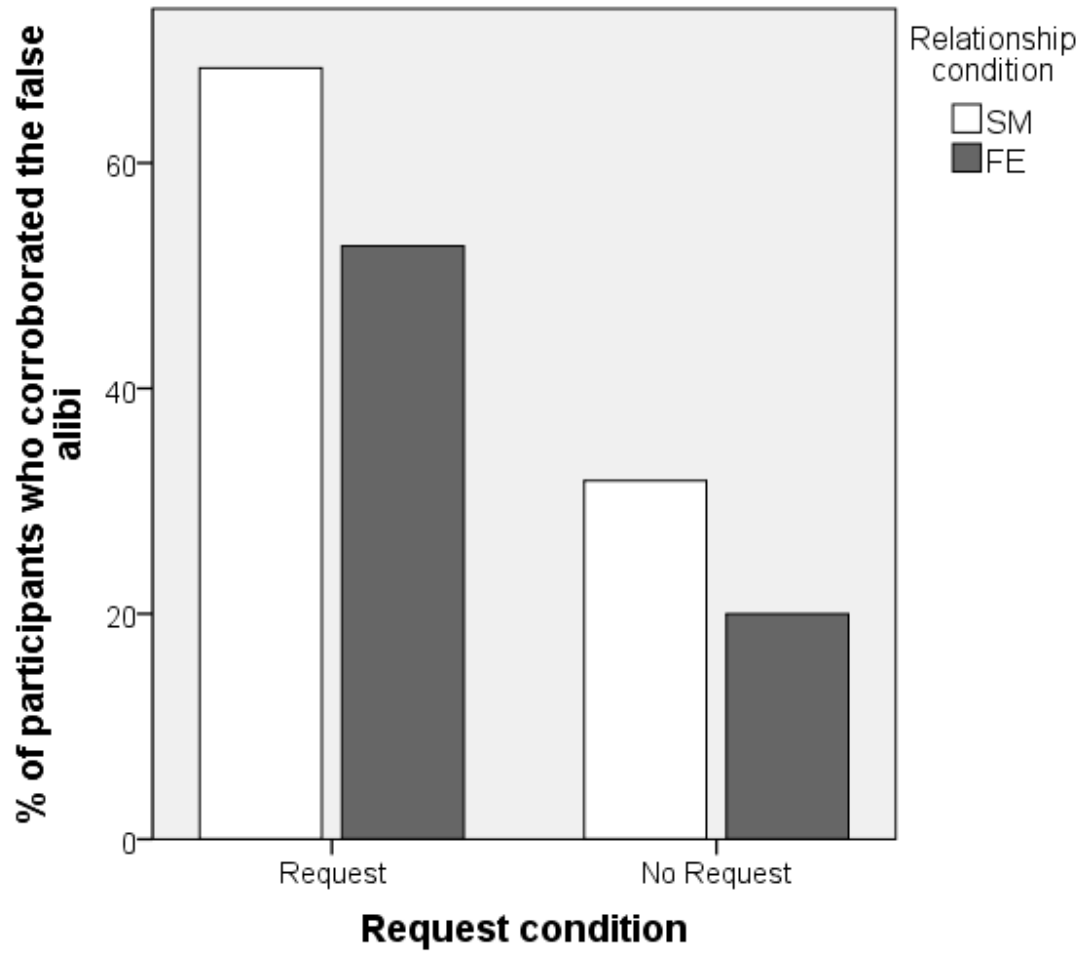

Figure 2. Percentage of participants who corroborated the confederate's false alibi, by relationship and request conditions 
Table 1.

Alibi Corroboration Contingency Table: Relationship Condition $\times$ Request Condition

\begin{tabular}{|c|c|c|c|c|c|c|c|}
\hline & & \multicolumn{2}{|c|}{$\begin{array}{l}\text { Participant did } \\
\text { not corroborate } \\
\text { alibi }\end{array}$} & \multicolumn{2}{|c|}{$\begin{array}{l}\text { Participant } \\
\text { corroborated } \\
\text { alibi }\end{array}$} & \multicolumn{2}{|c|}{ Total } \\
\hline & & $\mathrm{N}$ & $\%$ & $\mathrm{~N}$ & $\%$ & $\mathrm{~N}$ & $\%$ \\
\hline \multirow{3}{*}{$\begin{array}{l}\text { Friendship- } \\
\text { Enhancing } \\
\text { Condition }\end{array}$} & $\begin{array}{l}\text { Request } \\
\text { Condition }\end{array}$ & 9 & 47.4 & 10 & 52.6 & 19 & 100 \\
\hline & $\begin{array}{c}\text { No Request } \\
\text { Condition }\end{array}$ & 16 & 80.0 & 4 & 20.0 & 20 & 100 \\
\hline & Total & 25 & 64.1 & 14 & 35.9 & 39 & 100 \\
\hline \multirow{3}{*}{$\begin{array}{l}\text { Stranger- } \\
\text { Maintaining } \\
\text { Condition }\end{array}$} & $\begin{array}{l}\text { Request } \\
\text { Condition }\end{array}$ & 6 & 31.6 & 13 & 68.4 & 19 & 100 \\
\hline & $\begin{array}{c}\text { No Request } \\
\text { Condition }\end{array}$ & 15 & 68.2 & 7 & 31.8 & 22 & 100 \\
\hline & Total & 21 & 51.2 & 20 & 48.8 & 41 & 100 \\
\hline \multirow[t]{3}{*}{ Total } & $\begin{array}{l}\text { Request } \\
\text { Condition }\end{array}$ & 15 & 39.5 & 23 & 60.5 & 38 & 100 \\
\hline & $\begin{array}{l}\text { No Request } \\
\text { Condition }\end{array}$ & 31 & 73.8 & 11 & 26.2 & 42 & 100 \\
\hline & Total & 46 & 57.5 & 34 & 42.5 & 80 & 100 \\
\hline
\end{tabular}

association, among participants who corroborated the alibi, between request condition and alibi corroboration $(p=.07$, FET, $O R=6.0)$, the loglinear analysis described above was repeated without these participants. Once again, the final model retained only the request condition $\times$ alibi corroboration outcome interaction $\left(L R: \chi^{2}(4, N=65)=1.69, p=\right.$ .79). There was a significant interaction between request condition and alibi corroboration, $\chi^{2}(1, N=65)=10.62, p=.001, O R=5.58$, but again there was no association between relationship condition and alibi corroboration $(p=.21)$ and no threeway interaction $(p=.78)$. This suggests that participants' behaviour did not change based 
on whether they reported remembering the confederate say the alibi, or not. In fact, a simple chi-square test of the relationship between participants' recall of the alibi and their corroboration revealed no association at all, $p=.88, \varphi=.02$.

Similarly, participants were also asked during the debriefing interview to explain why they did or did not corroborate the confederate's alibi (see exploratory analyses section below). Despite the short time span between the confederate's absence and the alibi corroboration phase, six participants who corroborated the alibi reported having done so because they forgot that the confederate had left (two in the FE condition and four in the SM condition). I again suspect that at least some of these responses were selfserving (see discussion section), however to ensure that no effect was obliterated by analyzing data containing non-genuine corroborations, the loglinear analysis was replicated without these six participants. Once again, the final model retained only the request condition $\times$ alibi corroboration outcome interaction $\left(L R: \chi^{2}(4, N=74)=2.01\right.$, $p=.73)$. There was a significant interaction between request condition and alibi corroboration, $\chi^{2}(1, N=74)=12.96, p<.001, O R=6.09$, but again there was no association between relationship condition and alibi corroboration $(p=.34)$ and no threeway interaction $(p=.67)$.

In order to further explore a possible association between relationship and false alibi corroboration, liking questionnaire scores were entered as a predictor in a logistic regression analysis, to see if how much participants liked a confederate, regardless of condition assignment, had an influence on whether they corroborated his or her false 
alibi. Liking scores, however, did not significantly predict alibi corroboration, $\beta=-0.02$ $(S E=0.02), p=.38, O R=0.99, \mathrm{CI}_{.95}[.95,1.12] .^{4}$

\section{Exploratory Analyses}

Suspiciousness of the confederate. Participants were asked during the debriefing interview to rate how suspicious they were that the confederate was guilty of stealing the money (on a 10-point Likert scale). Participants' ratings ranged from 1 to 8, with a mean of $2.78(S D=1.96)$. It was possible that the confederate's request to keep his or her absence from the experimenter may have increased participants' suspicion of the confederate's guilt. It was also possible that due to a halo effect, participants in the FE conditions would be less suspicious of the confederate than participants in the SM condition. To see if any of the experimental manipulations affected participants' suspiciousness, a 2(relationship condition) $\times 2$ (request condition) ANOVA on participants' suspiciousness ratings was conducted. There was no significant main effect or interaction, all $p s>.87$, all $\eta_{p}^{2}<.001$.

Exploring predictors of false alibi corroboration. Although suspiciousness of the confederate was not affected by the request and liking manipulations, it was possible that those who were more suspicious of the confederate to begin with were less likely to corroborate the false alibi (Marion \& Burke, 2013). Thus, the potential predictive ability of suspiciousness ratings beyond request condition was investigated with a hierarchical

\footnotetext{
${ }^{4}$ Again, non-significant results were obtained when excluding participants who reported not remembering that the confederate had left the room, $\beta=-0.02(S E=0.02), p=.37$, $O R=0.98, \mathrm{CI}_{.95}[.95,1.02]$.
} 
logistic regression analysis (with request condition entered first). ${ }^{5}$ However, only request condition significantly predicted corroboration decision, $\beta_{\text {request }}=1.46(S E=0.48)$, $p=.002, O R=4.32, \mathrm{CI}_{.95}[1.68,11.15]$, model $\chi^{2}(2, N=80)=9.83, p=.007, R^{2}=.09$ (Hosmer \& Lemeshow), .12 (Cox \& Snell), .16 (Nagelkerke). Residual and collinearity statistics were all adequate. Suspiciousness ratings did not significantly predict alibi corroboration, $\beta_{\text {suspiciousness }}=-0.02(S E=0.12), p=.89$, OR $=0.98, \mathrm{CI}_{.95}[.77,1.25]$.

Reasons for corroboration or rejection of the alibi. During the debriefing interview participants were asked to explain why they did or did not corroborate the confederate's alibi (debriefing questions 4 and 6). Appendix $\mathrm{C}$ shows a breakdown of participants' reasons for either corroborating the alibi or rejecting the alibi, by relationship and request conditions (responses from 79 participants are shown, since one participant declined the debriefing interview), and category descriptions for the responses are outlined in Appendix B. ${ }^{6}$

Of the 46 participants who did not corroborate the alibi, the most commonly reported explanation was because of a belief that it was the right thing to do ('Moralitybased response', 38 or $83.6 \%$ of participants who did not corroborate), because of a lack of trust or personal knowledge of the confederate (12, or $26.1 \%)$, and because of a belief that going to the washroom was an irrelevant and innocuous event and could be reported (9, or 19.6\%). A higher percentage of participants in the SM than in the FE condition

${ }^{5}$ Although there is evidence suggesting that age may predict the tendency to lie (e.g., Serota et al., 2010), age was not a viable predictor in this case due to the very limited age range of our sample ( $>70 \%$ of participants were either 18 or 19 years of age).

${ }^{6}$ Due to the large number of low frequencies, non-independence of observations, as well as the exploratory nature of these data, only descriptive statistics are reported. 
(38.1\% vs. $16.0 \%$ ) and a higher percentage of participants in the NR than in the request condition (35.5\% vs. $6.7 \%$ ) said that they had a lack of trust in the confederate. Finally, more participants in the NR than in the request condition reported that the confederate's absence was irrelevant and innocuous (25.8\% vs. $6.7 \%)$. Other responses from participants who did not corroborate the alibi included trusting that the confederate was innocent (6 participants), simply responding to the experimenter ('Compliance', 6 participants), wanting to avoid a confrontation or involvement in the situation (2 participants), and being caught off guard without time to reflect on the situation (1 participant).

Of the 33 participants who did corroborate the alibi, the most common reasons for doing so were to protect the confederate or protect a relationship with the confederate (16 or $48.5 \%$ of participants who corroborated), because the participant trusted that the confederate was innocent (11, or 33.3\%), and because of a belief that going to the washroom was an irrelevant event that did not need to be reported (10, or 30.3\%). More participants in the request than in the NR condition reported wanting to protect the confederate or their relationship with him or her (56.5\% vs. $30.0 \%)$. Other reported reasons for corroborating the alibi included because the confederate asked them not to report their trip to the restroom (9 participants) a wish to avoid a confrontation or involvement in the situation (7 participants), because they forgot that the confederate left (6 participants), or because they were caught off guard (3 participants). (See Appendix C for a complete summary of participant responses. 


\section{Discussion}

Contrary to what I predicted, increasing the degree to which a participant liked a confederate did not increase the likelihood that the former would lie by corroborating the confederate's false alibi. A request from confederates to not disclose their whereabouts, however, did have an impact on participants' decision to corroborate the confederate's false alibi. Participants were much more likely to corroborate the alibi when they were asked not to reveal the truth, regardless of how much participants liked the confederate. In addition to examining the effects of the two main independent variables on corroboration rates, participants' suspiciousness of the confederate's guilt was also examined as a predictor of false alibi corroboration. Although manipulating how guilty a confederate appears has been shown to decrease the likelihood of corroborating a false alibi (Marion \& Burke, 2013), the natural variability of participants' suspiciousness of the confederate in this study was not associated with alibi corroboration likelihood. Finally, participants' explanations of their decisions to either corroborate the confederate's alibi or not offer some insight into some participants' motivations to lie or to tell the truth.

\section{Liking and Alibi Corroboration}

Hypothesis 1 stated that participants who underwent liking-enhancement manipulations with a confederate would be more likely to lie for him or her by corroborating a false alibi compared to those whose liking of the confederate was not increased. This manipulation was a direct replication from Marion and Burke (2013). The null findings obtained in the original study were largely unexpected given the existing literature on the psychology of alibis that suggests that those who are close to one another report being more willing to lie for one another (e.g., Hosch et al., 2011) and 
are expected to lie more frequently (e.g., Olson \& Wells, 2004). However, the same null effect was found in this study. In fact, participants in the stranger-maintaining condition were somewhat more likely to corroborate the confederate's false alibi (49\%) than participants in the friendship-enhancing condition (36\%, although this difference was not statistically significant). These results were once again obtained despite an apparent successful manipulation of liking as evidenced by the strong effect of relationship condition on participants' Liking scores. This effect also seemed to last the duration of the study, since liking ratings obtained at the debriefing interview were still significantly higher in the FE condition than in the SM condition.

It is true that previous relevant research on alibi corroboration always considered actual relationship difference as opposed to liking, but at a minimum, pairs in this study's FE condition would have been akin to emerging friends, acquaintances, or coworkers (i.e., two students in the same class who are planning to study together in the near future). Even among distantly-related individuals (familiar stranger vs. co-worker) there is an expectation that social distance will influence altruistic lying in an alibi corroboration context. For example, participants in Hosch et al. (2011) estimated that a complete stranger would corroborate a false alibi $6 \%$ of the time, a familiar stranger $15 \%$ of the time, and a co-worker $31 \%$ of the time (and reports of participants' willingness to lie for these same individuals mirrored these estimates). In this study, however, alibi witnesses in the FE conditions (who could be considered co-workers or acquaintances) lied for the suspect $36 \%$ of the time and alibi witnesses in the SM condition (who could be considered complete strangers or familiar strangers) lied $49 \%$ of the time. In light of these numbers, as well as those reported in Marion and Burke (23\% in both relationship 
conditions, 2013), it seems not that friends' (or co-workers') altruistic lying is overestimated, but that strangers' rate of altruistic lying is underestimated. The high corroboration rate in this study is undoubtedly due to half of the participants who were asked to withhold the truth, but the percentage of participants corroborating the false alibi in the NR/SM condition (32\%) is still much higher than the likelihood estimated in selfreport studies (e.g., Hosch et al., 2011). In addition, in real-life scenarios, a request to lie from the suspect is probable (and is likely assumed in hypothetical scenarios), making the

discrepancy between estimated and actual behaviour even more glaring. Despite the lack of an effect of relationship condition on alibi corroboration found in this study, it is still possible that more authentic differences in affinity between a suspect and an alibi witness (i.e., true friends or family members) can influence the witness' willingness to lie, but that the manipulations used did not create two groups distinct enough in affinity to show an effect (this was investigated in study 3 ).

\section{Compliance and Alibi Corroboration}

Hypothesis 2 stated that those who were explicitly requested not to tell the experimenter that the confederate went to the restroom would be more likely to corroborate the false alibi that he or she was in the testing room the whole time than those who were not asked to withhold this information. This hypothesis was supported: Participants were over twice as likely to corroborate the confederate's false alibi when the latter made an explicit request to withhold the truth than when no such request was made (61\% vs. $26 \%)$. This behaviour can easily be construed in terms of compliance with a request. An explicit request to lie (or at a minimum to not report the truth), as opposed to an implicit one made by simply stating the false alibi in the participant's 
presence, increased rates of compliance by more than half. The explicit request made by the confederate may have increased participants' perceived responsibility and motivation to 'help', thereby increasing the rate of corroboration (Cialdini \& Goldstein, 2004). It is important to note that the situation that participants were in was an ambiguous one: they had no objective knowledge of whether the confederate was guilty or not and the confederate did not ask them to lie for them in order to cover up a crime. He or she simply asked them to withhold some information that could (maybe) get them in trouble should the experimenter find out (i.e., the experimenter might get upset if she knew a study participant left without permission during the study session). Had the request been explicit in its aim to conceal a crime, the corroboration rate in the request condition may have been significantly lower. As suggested in the last section, a suspect requesting an alibi witness to lie (or withhold the truth) is likely a more realistic scenario than the absence of such a request. However whether suspects (innocent and guilty) take advantage of creating ambiguous situations (e.g., by implying a motivation to hide a negative albeit non-criminal behaviour) is currently unknown.

\section{The Role of Memory}

An unanticipated response from several participants in this study was that they did not report the confederate's absence, and instead corroborated the false alibi, because they had forgotten that he or she had left. This was surprising because they were claiming that they had forgotten an event that occurred at most 10 minutes earlier. One possible explanation for this response is that it is self-serving. Participants provided these responses after being told that their corroboration of the confederate's false alibi was the main measure of the study, and after realizing that the experimenter was aware of their 
lie. When asked to explain why they did not report the confederate's absence, a socially acceptable answer would be that they forgot that the participant left, thus no longer making their corroboration a lie.

Another possibility is, of course, that these participants actually did forget that the confederate had left. It is possible, for example, that the task they were engaged in (rating abstract images on physical attractiveness) was cognitively demanding enough for them not to notice the participant announce that he or she was going to the washroom, leave the room, and re-enter the room 3 minutes later. As unlikely as this seems, the data do provide some anecdotal evidence for genuine forgetting. For example, one participant initially corroborated the alibi when the experimenter asked her for a confirmation, but reported the absence at the very beginning of the debriefing, before being informed of the study's purpose. Another reason to suspect that forgetting did occur is that participants were more likely to report that they had corroborated the alibi due to forgetting if they were in the no request condition than if they were in the request condition $(40.0 \%$ vs. $8.7 \%$, although these statistics were based on small cell sizes with rather small expected frequencies). It is possible that the request itself emphasized the confederate's absence and acted as a reminder of the event, making it less likely for those participants to forget it. Without question, if an alibi witness can be mistaken about a suspect's whereabouts 10 minutes earlier, then it is reasonable to assume that an alibi witness' accounts for an event days or months in the past may be vulnerable to memory errors.

This last point, however, does also highlight a limitation of this study: The salience of the confederate's absence was confounded with the request condition. By requesting that the participant not report their absence to the experimenter, the 
confederate essentially 'reminded' participants in this condition that he or she had just left, whereas no such 'reminders' were included in the no request condition. However, if this difference does in fact affect memory, then one would expect more corroborations (due to forgetting) in the no request than in the request condition, which is the opposite from the pattern found. In addition, this difference in absence salience due to an explicit request would also be expected in real-life cases, thus eliminating this confound may reduce the external validity of the study.

\section{Reasons for Lying and Telling the Truth}

When participants were asked to explain why they did or did not report to the experimenter that the confederate had left the room, several recurrent themes were observed. A full summary of these responses can be found in Appendix C, but a few observations are worth highlighting. Interestingly, some explanations were offered as rationales for both lying and telling the truth. For example, $20 \%$ of participants who did not corroborate the alibi reported doing so because the confederate simply went to the restroom, which is an irrelevant and innocent behaviour that does not need to be concealed. Similarly, $30 \%$ of participants who corroborated also reasoned that going to the restroom is irrelevant and of little importance to the situation, but for that reason does not need to be reported. Another common response given by both those who corroborated and those who did not was believing that the confederate was innocent,

allegedly contributing to $33 \%$ of corroborators' decisions (e.g., "I don't believe she stole the money, so why make her appear suspicious by reporting that she left?") and $13 \%$ of non-corroborators' decisions (e.g., "I don't believe that she stole the money, so why lie about her going to the washroom?"). Thus, it appears that high trust in a suspect and a 
failure to appraise his or her behaviour as suspicious and potentially incriminating may lead to either lying or truth telling.

Also noteworthy was the lack of any discernable differences in participants' reasons for corroborating the confederate's alibi between those in the FE condition and those in the SM condition. This is in line with the lack of effect of relationship condition on corroboration rates. Those who did corroborate the confederate's false alibi did so for similar reasons, regardless of their affinity to the confederate. Close others have been called 'motivated others' in the psychological literature on alibis because these individuals are assumed to be motivated to lie for one another whereas strangers are assumed not to have such motivations. Such differences in motivations, however, were not found. Thus, the assumption may be incorrect, or, as alluded to above, the liking manipulation used in this study may not have been strong enough to engender the same kinds of motivation that a real friend or relative would naturally have to protect a suspect.

Finally, of the participants in the request condition who corroborated the confederate's alibi, 35\% admitted doing so because of the confederate's request, and this number accounts for the difference in corroboration rates between those in the request condition and those in the NR condition. Ironically, a difference that one would assume to exist between relationship conditions was present only between request conditions: $57 \%$ of participants in the request condition said they corroborated the alibi to protect either the confederate or their relationship with the confederate, versus $30 \%$ in the NR condition. Thus, it seems that the request directly influenced participants' decisions to corroborate the alibi. 
In this study, a direct request to withhold a confederate's whereabouts during the time of the crime largely increased the odds that a participant would lie to the experimenter. Thus, an explicit increase in the social pressure to conceal the confederate's absence led to more instances of false alibi corroboration. However, it is unclear whether a less conspicuous increase in social pressure could also influence an alibi witness' decision to lie. By modifying the experimental paradigm, I investigated in study 2 whether a reciprocity norm created by a confederate helping participants would create the social pressure required to increase the rate of corroboration compared to when a confederate did not help participants. Also included in study 2 were two personality measures to examine any possible associations between personality characteristics and propensity to corroborate a false alibi, and an exploration of gender differences by recruiting a sufficient male sample to compare corroboration between males and females. 


\section{Study 2 - The effect of gender and prior help on corroboration of a false alibi}

Under the pretext of a problem-solving study investigating the role of personality similarity on performance, male and female participants interacted with a confederate of the same gender. During the first part of the experimental session, help toward the participant was manipulated. In the 'prior help' condition the confederate helped the participant complete a difficult task, which increased the participant's chances of winning a prize. In the 'no prior help' condition, the confederate did not help the participant complete the difficult task. During the second part of the experimental session, all participant-confederate pairs completed a collaborative task. During this task the confederate momentarily left the study room under pretext of needing to use the restroom. Following the task the experimenter questioned the dyad about a theft that had just occurred, for which the confederate offered a false alibi. The participant was later given the opportunity to either corroborate the confederate's alibi that he or she remained with the participant during the time of the crime, or to refute it. The design of this study was a 2 (prior help condition) $\times 2$ (gender) between-subjects factorial. The main dependent variable was the participant's corroboration or non-corroboration of the confederate's alibi.

\section{Participants}

Eighty-five participants took part in the study. All participants were undergraduate students and each received either one course credit or ten dollars as compensation for their participation. Their age ranged from 18 to 38 years with a mean of 21.53 years $(S D=4.26)$. The study was advertised on SONA under the title "Does 
Personality Affect Group Problem-Solving?" for introductory psychology students, as well as via flyers posted around the university campus for non-psychology majors (see Appendix D for all material used in study 2). Both males and females were recruited separately on SONA, and non-psychology students were scheduled to participate with a gender-matched confederate. Efforts were made to recruit enough male participants to allow for gender comparisons.

Four participants were dropped from the data set and subsequent analyses due to their strong disbelief in the cover story, as became evident during the debriefing session. Another participant was excluded due to a confederate's error in timing of when he left the study room. Thus, the data from a final sample of 81 participants ( 44 females) were retained for analyses.

\section{Setting and Apparatus}

This study took place in the same setting as study 1 , and the same equipment was used, with the exception that the audio-recorder was not used in this study.

\section{Measures}

Liking Questionnaire. Participants were asked to complete a modified version of the Liking questionnaire used in study 1: Five of the 10 Likert-type items were used, for the sake of brevity. Because it was possible that those in the 'prior help' condition would come to like the confederate more than those in the 'no prior help' condition, the liking score was used as a potential covariate to isolate the effect of prior help on alibi corroboration. The Liking questionnaire items were embedded amongst other 
demographic questions (age and gender) and filler questions relating to the problemsolving task.

Revised Social Responsibility Scale (SRS). Participants were asked to complete the Revised Social Responsibility Scale (Berkowitz \& Daniels, 1964), a measure adapted for college students from Harris' (1957) scale for children. According to Berkowitz and Daniels (1964), the social responsibility norm should be more salient for participants who received help from the confederate than those who did not. Thus, this measure served as a check for the effectiveness of the helping manipulation as a way to increase participants' awareness of the social responsibility norm.

Right-Wing Authoritarianism (RWA) scale. Participants completed the RightWing Authoritarianism scale (Altemeyer, 1981). This scale measures the extent to which individuals ascribe to conservative values and unequivocally trust and submit to political leaders and authority figures (Altemeyer, 1981), and was included as an exploratory measure. It is possible that those participants who score higher on authoritarianism would be less willing to lie to an authority figure (i.e., the experimenter) and thus less likely to corroborate a false alibi.

Ten-Item Personality Inventory (TIPI). Participants completed the Ten-Item Personality Inventory (TIPI, Gosling et al., 2003), a brief measure of the 'Big Five' personality dimensions. This scale has been empirically validated, with adequate psychometric qualities (although of somewhat lesser quality than full length personality measures, the brevity of this scale was preferred as it was included for exploratory purposes only). 
Post-Measure Questionnaire. Participants again answered a filler questionnaire at the end of the experimental session. It was administered in order to provide an opportunity for the participant and confederate to be separated and for the experimenter to question the participant about the confederate's alibi (see Procedure section, Study 1).

Debriefing Questionnaire. The same debriefing questionnaire used for study 1 was used for study 2, with the exception of the manipulation check questions relating to the request and liking manipulations. Again, participants were asked to explain why they did or did not corroborate the confederate's alibi.

\section{Procedure}

The experimental paradigm was similar to the one used in Study 1. The first part of the experimental session (the 'Word generation task' and 'Liking questionnaire' phases) was modified to accommodate the helping manipulation. The second part of the experimental paradigm (the 'Restroom break', 'Mock theft and false alibi', 'Alibi corroboration or rejection', and 'Debriefing interview' phases) was identical to the 'No Request' condition of study 1 , with the exception that different filler tasks were completed during the 'Restroom break' phase.

Participants were randomly assigned to one of two helping conditions: prior help $(\mathrm{PH})$ or no prior help $(\mathrm{NH})$. The experimenter was blind to the helping condition assignment until the end of each session. The confederates, however, had access to the condition assignment list and thus were able to act accordingly for each session.

From the SONA study posting, participants were informed that the purpose of the study was to investigate how personality affects performance on problem solving when 
people work individually and together with someone else. Participants were tested in pairs, however the second 'participant' was a confederate. The participant and confederate were scheduled to arrive at the laboratory at the same time, and were presented with consent forms to read and sign. They were told that they would be asked to solve sets of problems, some individually and some together, and answer some questionnaires and personality measures. The participant and confederate completed all problem-solving tasks while they were in the same study room, and all questionnaires they completed individually in separate rooms. The experimenter provided the necessary materials at the start of each phase, but was not present during their completion.

Individual Problem-Solving Task. The participant and confederate were each given the same paper-and-pencil package containing a set of ten problems (a mix of anagrams, math and probability, pattern recognition, and logical reasoning problems) to be completed individually. They were given eight minutes to solve as many problems as they could, and the experimenter informed them that participants who answered at least six problems correctly would be entered into a draw to win a \$50 gift card to Milestones Restaurant. The task was designed, based on pilot data, so that it would be very difficult to actually obtain six correct answers in the time given to solve them, however participants were encouraged to guess if they were unsure of the answer to make sure all had answered at least six problems. In the PH condition, while working individually but in the same room, the confederate gave the participant the answers to three of the most difficult problems (\#s 6, 9, and 10 - answers that participants were unlikely to have obtained). To minimize participants' suspicion of the confederate's identity, the confederate stated that he or she had recently completed the same problems for another 
study. If participants asked for additional answers, confederates were instructed to provide them. In the NH condition, the confederate worked on his or her own package without helping the participant, but instead asked the participant for the answer to a difficult problem (\#6, which was never given). This was done as an attempt to match the level of interaction in the two conditions, as well as to make the confederate appear as willing to 'break the rules' in the $\mathrm{NH}$ condition as in the $\mathrm{PH}$ condition. If participants asked the confederate for answers, the latter was instructed to pretend not to know them. At the end of the eight-minute period, the experimenter instructed one dyad member (the confederate) to go to a different room to complete the next part of the study.

Personality Questionnaires. The participant and confederate were separated for this phase, under pretext that the answers to the personality questionnaires must remain confidential. The participant completed the Liking questionnaire, the TIPI, the RWA scale, and the Revised SRS. Shortly after starting these questionnaires, the experimenter informed the participant that he or she had made it into the draw for the gift card.

Restroom Break. Upon completion of the personality questionnaires, the confederate was returned to the study room. The procedure for this phase was the same as in the 'No Request' condition of Study 1, except that the tasks consisted of problems similar to those in the individual problem-solving task. The pair was asked to solve, in order, a set of quantitative, pattern recognition, and logical reasoning problems presented on a laptop computer. The experimenter provided all material and instructions for the three tasks. The pair was told that the pattern recognition task was being piloted, and that a physiological measure was required from the person completing it. Again, since only one heart rate monitor was available, the participant was told that only one person could 
complete this task and that he or she was randomly selected for it. The monitor was attached to his or her finger at the start of this phase, before the experimenter left. It was during the pattern recognition task that the confederate left to use the restroom.

All subsequent procedure for the Restroom break phase, as well as those for the Mock theft and false alibi, Alibi corroboration or rejection, and Debriefing interview phases were identical to those in Study 1 (NR condition), with the single exception of an additional set of questions asked to participants who did not corroborate the confederate's alibi. If a participant revealed that the confederate left to go to the restroom, the experimenter asked him or her 1) to estimate the duration of the confederate's absence, and 2) the direction in which the confederate turned (right was in the direction of the washroom, and left was in the direction of the office where the mock theft took place, although because the confederate always closed to door behind him or her it would be impossible for participants to know). This was done to determine whether participants in the $\mathrm{PH}$ condition would try to divert the blame away from the confederate by reporting shorter absence duration and/or a direction of travel away from the location of the theft. At the end of the study, all participants were debriefed. Again, no participant expressed concerns over the deception used, and the vast majority stated that they enjoyed the study.

\section{Results}

\section{Experimental Manipulation Checks}

Prior Help, Reciprocity, and Liking. The main reason that help was manipulated was in the hopes that this would increase the salience of the social 
responsibility norms in participants in the $\mathrm{PH}$ condition, as compared to those in the $\mathrm{NH}$ condition. This was measured using Berkowitz and Daniels' (1964) 22-item Revised Social Responsibility Scale (SRS), which has previously been shown to correlate with prior help. Two participants had missing ratings for either one or two items on this scale, for which person-mean imputations were used (Downey \& King, 1998; Hawthorne \& Elliott, 2005). One participant's score was excluded from this analysis due to having too many unanswered scale items (41\%). SRS scores across all participants ranged from 71 to 109 with a mean of 86.56 (out of a maximum score of $110, S D=7.61$ ). Surprisingly, a 2 (help condition) $\times 2$ (gender) ANOVA on participants' SRS scores revealed no effect of help condition, $p=.69, \eta_{p}^{2}<.01$ (PH: $M=86.92, S D=8.69$; NH: $M=86.21, S D=6.44$ ), but a main effect of gender, $F(1,76)=5.56, p=.02, \eta_{p}{ }^{2}=.07$. Females had significantly higher SRS scores $(M=88.37, S D=8.35)$ than did males $(M=84.46, S D=6.10)$. The interaction between help condition and gender was not significant, $p=.25, \eta_{p}{ }^{2}=.02$.

Although many participants in the PH condition took the three answers given by the confederate $(41.5 \%)$, others refused some or all of the answers (22\% took 2, 17.1\% took 1 , and $7.3 \%$ took none), and others asked for more than the three offered (9.8\% took 4 and $2.4 \%$ took 5). It was therefore possible that SRS scores varied as a function of how many answers participants took from the confederate (i.e., the more answers they obtained from the confederate, the greater their chances of being entered into the gift card draw). However, the number of answers obtained was not correlated with SRS scores, $r_{s}=-.03, p=.41$.

Another potential effect of the helping manipulation could have been an increase of participants' liking of the confederate. Participants who were helped may have liked 
the confederate more than participants who were not helped. A shortened, 5-item Liking questionnaire was administered for this purpose, which had good internal reliability (Cronbach's $\alpha=.80$ ), with all item-total correlations ranging from .55 to .62 (Nunnally, 1978). Liking scores across all participants ranged from 15 to 35 , with a mean of 25.6 (out of a maximum score of $35, S D=4.73$ ). A 2 (help condition) $\times 2$ (gender) ANOVA revealed a main effect of help condition, $F(1,77)=17.01, p<.001, \eta_{p}^{2}=.18$. Participants who were helped liked the confederate more $(M=27.56, S D=4.04)$ than participants who were not helped $(M=23.60, S D=4.58)$. There was no significant effect of gender, $p=.19, \eta_{p}{ }^{2}=.02$, and no interaction between helping and gender, $p=$ $.90, \eta_{p}{ }^{2}<.01$. Thus, in comparison to those in the $\mathrm{NH}$ condition, participants in the $\mathrm{PH}$ condition did not seem to experience an increase in feelings of social responsibility, but they did show an increase in confederate liking.

Finally, because helping participants entailed the confederate essentially cheating on the problem-solving task, and because suspiciousness may impact corroboration rates (Marion \& Burke, 2013), it was possible that participants in the PH condition would question the confederate's moral fiber, and in turn be more suspicious of him or her. Participants were asked during the debriefing interview to rate how suspicious they were of the confederate's involvement in the theft. A 2 (help condition) $\times 2$ (gender) ANOVA on suspiciousness ratings revealed no main effect and no interaction, all $p \mathrm{~s}>.43$, all $\eta_{p}{ }^{2}<.01$. Thus, efforts made to avoid this potential confound by having confederates in the $\mathrm{NH}$ condition attempt to cheat (by asking the participant for answers) seem to have been successful. 
Attention to alibi. As was done in study 1, confederates in study 2 were instructed to make their alibi as obvious as possible by making eye contact with the participant, and repeating it if necessary. Despite these best efforts, a substantial portion of participants (39.5\%) again reported during the debriefing interview that they could not recall the alibi. This did not have any impact on the results in study 1 , but again as a precaution, the main analyses were conducted with and without these cases.

\section{Main Analyses}

Twenty-five out of 81 participants $(30.9 \%)$ corroborated the confederate's alibi by confirming that the latter had remained in the study room during the time of the theft. Of those participants who truthfully reported that the confederate had gone to the restroom, the large majority did so when the experimenter asked them to confirm the alibi. Two participants reported the confederate's absence after the confederate had left the study room but before the experimenter questioned them, and the remaining 3 participants informed the experimenter of the absence at the start of the debriefing interview (after initially corroborating the alibi when questioned, but before they were informed of the true purpose of the study).

A three-way hierarchical loglinear analysis with help condition, gender, and alibi corroboration outcome was conducted. Although the final model did not retain the threeway interaction, there was a non-significant association between gender and alibi corroboration, $\chi^{2}(1)=2.99, p=.08, O R=2.32$. Contrary to what was predicted, male participants were 1.78 times more likely to corroborate the confederate's false alibi than female participants (40.5\% vs. $22.7 \%$, see Figure 3$)$. Also contrary to my prediction, participants in the $\mathrm{PH}$ condition were not more likely than those in the $\mathrm{NH}$ condition to 
corroborate the alibi ( $31.7 \%$ vs. $30.0 \%$, respectively), $p=.87$. Finally, there was no interaction between help condition and gender on alibi corroboration, $p=.89$ (and none was predicted, see contingency table, Table 2). Thus, neither hypothesis 3 nor hypothesis 4 was supported.

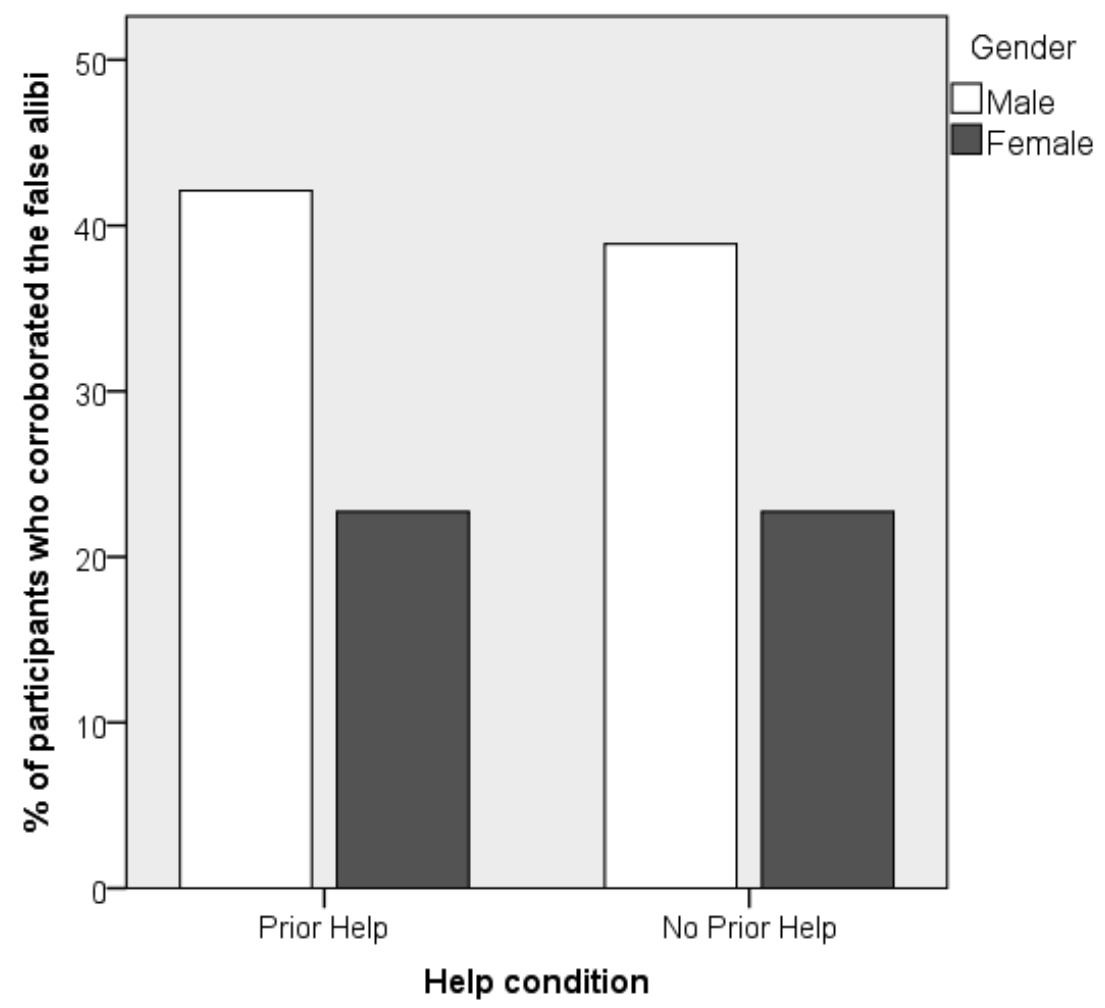

Figure 3. Percentage of participants who corroborated the confederate's false alibi, by help condition and gender

Because the helping manipulation seemed to affect participants' liking of the confederate, liking scores were entered into a logistic regression analysis to investigate their ability to predict the probability of corroboration. Not surprisingly, however, given the results in study 1 , liking scores did not significantly predict corroboration, $\beta=0.02$ $(S E=0.05), p=.65, O R=1.02, \mathrm{CI}_{.95}[.93,1.13]$. 
Since many participants reported not having heard the confederate's alibi (or could not remember the confederate saying it), analyses testing the main hypotheses were repeated without these participants. Because of the decrease in sample size $(N=49)$, the number of cells with expected frequencies below 5 rose to above $20 \%$, thus the data did not meet the assumptions necessary for a three-way loglinear analysis. Instead, separate

Table 2.

Alibi Corroboration Contingency Table: Help Condition $\times$ Gender

\begin{tabular}{ccc|ccc|cc}
\multirow{2}{*}{$\begin{array}{c}\text { Prior Help } \\
\text { Condition }\end{array}$} & Males & $\begin{array}{c}\text { Participant did } \\
\text { not corroborate } \\
\text { alibi }\end{array}$ & \multicolumn{2}{c|}{$\begin{array}{c}\text { Participant } \\
\text { corroborated } \\
\text { alibi }\end{array}$} & \multicolumn{2}{|c}{ Total } \\
\cline { 3 - 9 } & Females & 11 & 57.9 & 8 & 42.1 & 19 & 100 \\
& Total & 28 & 68.3 & 13 & 31.7 & 41 & 100 \\
\hline No Prior Help & Males & 11 & 61.1 & 7 & 38.9 & 18 & 100 \\
Condition & Females & 17 & 77.3 & 5 & 22.7 & 22 & 100 \\
& Total & 28 & 70.0 & 12 & 30.0 & 40 & 100 \\
\hline Total & Male & 22 & 59.5 & 15 & 40.5 & 37 & 100 \\
& Females & 34 & 77.3 & 10 & 22.7 & 44 & 100 \\
& Total & 56 & 69.1 & 25 & 30.9 & 81 & 100
\end{tabular}

chi-square tests of the relationship between alibi corroboration and each of the two independent variables were computed. In line with the previous results, there was no association between help condition and alibi corroboration, $p=.92, \varphi=.01$. The association between gender and alibi corroboration this time was not significant, $p=.21$, $\varphi=.18$ (likely due to the loss of power), despite males again being almost twice as likely to corroborate the alibi as females ( $32.0 \%$ vs. $16.7 \%$, respectively). 
As in study 1, a subset of participants who corroborated the alibi (15 out of 25) stated that they had done so because they forgot that the confederate had left, and thus were not actually lying for him or her (see exploratory analyses below). Although there are reasons to believe that these answers were self-serving (see discussion section), the main hypotheses were tested without these participants in case the full data set does contain non-genuine corroborations. Again, separate chi-square tests were done due to low expected frequencies precluding a loglinear analysis. There still was no association between help condition and alibi corroboration, $p=.31, F E T, \varphi=.14$, and there remained an association between gender and alibi corroboration, and this time it reached significance, $p=.04, F E T, \varphi=.29$. Male participants were almost 5 times more likely to corroborate the confederate's false alibis than female participants (26.7\% vs. $5.5 \%)$.

Because there were only four individuals playing the role of confederates for this study - two males and two females - it was possible that the gender effect found was due to individual differences (which may have led participants lie less often for one female confederate or more often for one male confederate). To explore this possibility, a chisquare test of independence between confederate identity and alibi corroboration was computed, which suggested independence between individual confederates and rates of corroboration, $p=.14$, FET, $\varphi=.26$. However, one female participant had a slightly higher corroboration rate than one of the male participant (28.6\% vs. $27.3 \%$, respectively).

Although there were no differences in alibi corroboration rates between participants in the $\mathrm{PH}$ and $\mathrm{NH}$ conditions, in order to further explore a possible relationship between helping and social responsibility on alibi corroboration, SRS scores 
as well as the number of answers that the participant obtained from the confederate were entered as predictors in a logistic regression analysis. However, neither SRS scores, $\beta=0.03(S E=0.03), p=.31, O R=1.03, \mathrm{CI}_{.95}[.97,1.10]$, nor number of answers obtained, $\beta=0.07(S E=0.17), p=.69, O R=1.07, \mathrm{CI}_{.95}[.77,1.48]$, predicted alibi corroboration. ${ }^{7}$ Finally, because gender did seem to be associated with corroboration, and because SRS scores significantly differed by gender, there was a potential for SRS scores to mediate the relationship between gender and corroboration. However, when SRS scores were regressed on corroboration outcome, while controlling for gender, the partial effect of SRS scores was not significant, $\beta=0.05(S E=0.04), p=.12, O R=1.06$, CI.95 [.99, 1.13].

\section{Exploratory Analyses}

\section{Estimates of Absence Duration and Direction of Travel. When participants} did not corroborate the confederate's alibi (which was most of the time), participants were asked to estimate the duration of the confederate's trip to the restroom, and if they knew which way the confederate turned when he or she exited the room. These questions were asked to explore how many participants would provide information suggesting either guilt or innocence, and if this correlated with how much they liked the confederate and how suspicious they were of him or her. Many participants failed to provide answers to these questions. Thirteen participants (out of 56) did not provide a time estimate. Of those who did, the average reported duration was 3.02 minutes $(S D=1.17)$ and ranged from 1 to 6.5 minutes. A $t$-test revealed no differences in duration estimates between the

\footnotetext{
${ }^{7}$ Non-significant results were also obtained when excluding participants who reported not remembering that the confederate had left the room.
} 
two help conditions, $p=.69, d=0.12$. Duration estimates were not significantly correlated with either liking scores, $r_{s}=-.16, p=.32$, or participants' suspiciousness of the confederate, $r_{s}=.04, p=.80$. Most participants (48 out of 56) did not provide a direction of travel. Confederates were instructed to close the door behind them as they left the study room, thus participants could not have known which direction they turned. However, 6 participants stated that the confederate turned left, toward the office, and 2 participants stated that the confederate turned right, toward the restrooms. No comparison between groups was possible due to the low response rate of this question.

Exploring Predictors of False Alibi Corroboration. Several exploratory measures were collected to investigate their potential associations with corroboration rates. These included the 'Big Five' personality traits as measured by the TIPI (conscientiousness, openness, emotional stability, extraversion, and agreeableness, Gosling et al., 2003), as well as a measure of authoritarianism, as measured by the RWA $\left(\right.$ Altemeyer, 1981) ${ }^{8}$. It was possible that participants low in authoritarianism may be more likely to corroborate the confederate's alibi than participants whose RWA scores reflect highly trusting and submissive behaviour toward authority figures. These measures, along with participants' gender, and their ratings of how suspicious they were of the confederate, were included in a logistic regression analysis. ${ }^{9}$ Because of the highly exploratory nature of this analysis, a stepwise (backward) method was used to decrease the risk of a significant predictor obtained by chance. Participants' suspiciousness ratings

\footnotetext{
${ }^{8}$ Person-mean imputations were conducted to calculate the RWA score of three participants who each had one missing item. Two other participants were excluded from these analyses due to missing $30 \%$ or more of the scale's items.

${ }^{9}$ Once again, age variability was too restricted to include in the analysis (80\% of participants were between 18 and 22 years of age).
} 
was the only factor retained in the final model, $\beta_{\text {suspiciousness }}=-0.20(S E=0.10), p=.049$, $O R=0.82, \mathrm{CI}_{.95}[.67, .999], R^{2}=.04$ (Hosmer \& Lemeshow), .05 (Cox \& Snell), .07 (Nagelkerke), model $\chi^{2}(1, N=79)=4.23, p=.04$. Residual and collinearity statistics were all adequate. For every unit increase in suspiciousness rating, the odds of a noncorroboration increased by 1.22 .

Reasons for Corroborating or Rejecting the Alibi. As in study 1, participants were asked during the debriefing interview to explain why they did or did not corroborate the confederate's false alibi. Appendix E shows a break down of participants' responses by help condition and gender (category descriptions can be found in Appendix B). ${ }^{10}$

Of the 56 participants who did not corroborate the alibi the most commonly reported reasons for doing so were due to a belief that it was the right thing to do (44 participants, or $78.6 \%$ of participants who did not corroborate), because of a lack of trust or personal knowledge of the confederate $(15$, or $26.8 \%)$, and because participants were simply complying with the experimenter's request to confirm or refute a fact $(12$, or 21.4\%). A higher percentage of participants in the $\mathrm{PH}$ than in the $\mathrm{NH}$ condition $(39.3 \%$ vs. $14.3 \%)$ and a higher percentage of male participants than female participants $(36.4 \%$ vs. $20.6 \%$ ) reported the confederate's absence because of a lack of trust in the confederate. Other responses from participants who did not corroborate the alibi included wanting to avoid confrontation or involvement in the situation (8 participants), believing that the confederate was innocent (7 participants), being caught off guard when asked the

\footnotetext{
${ }^{10}$ Again, because of low cell frequencies, non-independence of observations, as well as the exploratory nature of this data, only descriptive statistics are reported.
} 
question ( 2 participants), and believing that going to the washroom was irrelevant to the situation and innocuous (1 participant).

Of the 25 participants who did corroborate the alibi, the most common reasons for doing so were due to forgetting that the confederate had left (15 participants, or $60 \%$ of those who corroborated the alibi), and because of a belief in the confederate's innocence (9, or $36 \%)$. Participants in the $\mathrm{PH}$ condition were more likely than those in the $\mathrm{NH}$ condition to report belief in the confederate's innocence as a reason for corroborating (53.8\% vs. $16.7 \%)$. Other reasons given for corroborating the false alibi were being caught off guard (6 participants), thinking that going to the washroom was not important to report (5 participants, 4 of which were male), to protect the confederate or their relationship with him or her (4 participants), and to avoid confrontation or involvement in the situation (1 participant). (See Appendix E for a full summary.)

\section{Discussion}

Contrary to predictions, having a confederate help a participant during a previous task did not subsequently increase the participant's likelihood of corroborating the confederate's false alibi, and female participants were not more likely to engage in altruistic lying by corroborating a false alibi. In fact, the opposite pattern was found for gender: male participants were more likely to corroborate the false alibi. In addition to the investigation of prior help and gender, participants' personality characteristics and their belief in the confederate's guilt were explored as predictors of false alibi corroboration. None of the personality characteristics measured (authoritarianism and the 'Big Five' factors) predicted corroboration outcome. Participant's suspiciousness of the 
confederate, however, did predict corroboration. Finally, participants' explanations for why they did or did not corroborate the confederate's false alibi mirror those obtained in study 1 and offer additional insight into common motivations for altruistic lying in an alibi corroboration context.

\section{Prior Help, Reciprocity, and Alibi Corroboration}

Help was manipulated in the hopes of influencing the salience of the reciprocity norm, which would, in turn, have increased or decreased the social pressure to help the confederate by corroborate his or her false alibi. Confederates manipulated help by either giving participants answers to difficult questions thereby increasing participants' chances of winning a prize in one condition, or not providing such help in the other condition. Unfortunately, this manipulation of help did not have the intended effect. Neither the experimental condition (help vs. no help) nor a measure of how much help was provided (as determined by the number of answers taken by the participant) was associated with whether or not the participant decided to lie for the confederate. However, because these two variables also did not correlate with SRS scores (used to infer salience of the reciprocity norm, see Berkowitz \& Daniels, 1964), it is possible that either the help manipulation used did not have the intended purpose of affecting the salience of the norm, or that the SRS scale failed to detect this change in motivation to reciprocate help. The help offered by the confederate may have not been important or obvious enough to produce in those helped a wish to 'return the favour'. For example, perhaps if the confederate's help ensured the participants would win a prize instead of only entering them in a draw it would have increased the significance of the help enough to induce reciprocal help toward the confederate. Another possibility is that the kind of help 
offered by the confederate was too different in nature from the kind of help involved in corroborating a false alibi. In most previous studies investigating the effect of help on reciprocity, the reciprocated act was identical (e.g., childcare, Davis \& Daly, 1997) or similar in nature (e.g., monotonous laboratory tasks, Daniel \& Berkowitz, 1963). Thus, it is possible that the reciprocity and social responsibility norms are best primed through similar behaviours (although see Greenglass, 1972). Perhaps a better help manipulation would have involved the confederate covering up for the participants (e.g., by taking the blame for a planned computer crash that appeared to be the participant's fault).

Interestingly, however, participants who scored high on the social responsibility scale (regardless of condition) were not more likely to corroborate the alibi. In fact, female participants, who scored significantly higher on the SRS, were less likely to corroborate the alibi (see next section). Thus, social responsibility as a trait does not appear to be associated with altruistic lying in an alibi corroboration context.

Regardless of its affect on the reciprocity norm, however, the help manipulation itself did occur. What this seemed to have done is simply increased the participants' liking of the confederate in the $\mathrm{PH}$ condition in comparison to the $\mathrm{NH}$ condition. Liking, however, as was the case in study 1 and in Marion and Burke (2013), was not associated with alibi corroboration.

\section{Gender and Alibi Corroboration}

Hypothesis 4 proposed that female participants would be more likely to corroborate a female's false alibi, compared to male participants corroborating a male confederate's alibi, because of evidence suggesting that women are more likely than men to tell other-oriented lies, and that people in general are more likely to lie for the benefit 
of women (e.g., DePaulo et al., 1996; Eagly \& Crowley, 1986; Vrij, 2008). However, the opposite pattern emerged: Male participants corroborated male confederates' false alibi almost twice as much as female participants corroborated female confederates' false alibi. Although there is little in the psychological literature on lying that would suggest that men in general lie more than women, very little research has been published on the kind of altruistic lying that is at play in a false alibi corroboration context. The original hypothesis was based on research findings that relate to the larger category of otheroriented lies, but altruistic lies tend to make up only a small portion of other-oriented lies (e.g., Camden et al., 1984). Thus, it is possible that the gender difference found in the frequency of other-oriented lie-telling may not hold up for altruistic lie-telling more specifically, or that the difference is reversed, as suggested here. A possible reason why altruistic lie-telling, specifically in the context of corroborating false alibis, could be more likely among men then among women is because not corroborating the alibi (i.e., telling the truth) can be considered 'snitching' on a peer. Although this 'anti-snitch' culture is most often talked about in relation to street, criminal, and prison communities (e.g., Huey \& Quirouette, 2010), the norm does seem to have permeated popular culture (as evidenced by a handful of participants who referred to it during debriefing), and there appears to be some evidence suggesting that the rule is more strongly enforced among men than among women (Huey \& Quirouette, 2010).

Yet another possibility is that participants make different attributions about male and female suspects. Although all of the confederates in this study were pleasant, friendly individuals, it is nevertheless possible that participants perceived male suspects as more intimidating (e.g. one of the confederates was an athlete of over six feet tall), and thus 
male participants were perhaps more likely to be concerned about seeing the suspect again following a non-corroboration. This leads to an interesting avenue for future research on the potential impact of confederate characteristics on alibi witness corroboration. However it also leads to another important consideration of the gender difference found in this study: It is difficult to interpret this effect not only because it is rather small and inconsistent, but also because of a partial confound between gender and confederate identity (since only two confederates of each gender were used). The fact that no significant differences were found in corroboration rates among the four confederates suggests that it was not a single male confederate or a single female confederate driving this effect, but at the same time there wasn't a clear dissociation: one female confederate and one male confederate had equal rates of corroboration. Thus, it is difficult to estimate the generalizability of the association. Still, potential gender differences should be considered in future research on alibi corroboration and altruistic lying more generally.

\section{Suspiciousness and Alibi Corroboration}

Of the several exploratory factors investigated as predictors of alibi corroboration, only participants' ratings of how suspicious they were of the confederate (i.e., how much they believed the confederate was guilty of the theft) was a significant predictor. Participants who were more suspicious of the confederate were less likely to lie for him or her by corroborating the false alibi. This sentiment was also reiterated in participants' reasons for why they did or did not corroborate the alibi: a lack of trust in the confederate was a common reason for telling the truth, whereas trusting the confederate was a common reason for lying for him or her (see section below on reasons for lying and 
telling the truth). This finding is also in line with results from Marion \& Burke (2013) who found that participants were much less likely to corroborate the false alibi of a suspect who was made to appear guilty than a suspect who appeared innocent. Although suspiciousness here was not manipulated, participants' variations in how the perceived the confederate and the situation seemed to determine how they reacted. This finding should be interpreted with caution, however, because suspiciousness was not manipulated. The ratings were obtained after the questioning phase (i.e., retrospectively), thus the directionality of the association is in question. That is, it is possible that the decision to either corroborate or reject the alibi influenced subsequent suspiciousness ratings.

\section{The Role of Memory}

There were again several participants in this sample who reported that they corroborated the confederate's alibi because they had forgotten that the confederate left to go to the restroom (and thus believed, at least momentarily, that the alibi was true). Whereas $18 \%$ of those who corroborated in study 1 claimed a lapse in memory, an astonishing $60 \%$ of alibi corroborators in this study reported forgetting the confederate's absence at the time that the experimenter asked them to confirm the alibi. As discussed in study 1, this likely reflects some self-serving answers (i.e., as a way to disguise a lie as an honest mistake), and some support for this interpretation comes from some participants providing other reasons, in addition to forgetting, for why they did not report the confederate's absence (e.g., because they trusted the confederate or didn't think it was important to report). However, there were again some instances in this study that appeared to reflect true forgetting. For example, three participants reported the 
confederate's absence just before the debriefing session, after they had initially corroborated. They all stated that they had remembered that the confederate had left while they were answering the last filler questionnaire, between the questioning phase and the debriefing session. Several other participants who corroborated the alibi also admitted during the debriefing that they also initially forgot but later remembered the confederate's absence during this questionnaire, but still opted not to report it (in which cases genuine false alibi corroborations can be inferred). One possible explanation for the much larger proportion of participants who 'lied' due to forgetting in this study, compared to study 1 , is that the filler task that participants were engaged in during the confederate's absence was much more cognitively demanding in this study than in study 1 (solving timed pattern recognition problems vs. rating the attractiveness of images). This explanation would also support the possibility of genuine forgetting.

\section{Reasons for Lying and Telling the Truth}

In addition to forgetting, participants provided several different reasons for why they did or did not report the confederate's absence when the experimenter asked them to confirm the false alibi, which were quite similar to the reasons provided by participants in study 1 (with the exception of corroborating because of the confederate's request). No participant reported lying for the confederate as a returned favour from the help received earlier in the study session. Several trends are consistent with those found in study 1. Again, both participants who corroborated the alibi and those who did not stated their decision was based on their trust that the confederate was innocent (36\% vs. 13\%). A trusting alibi witness could then lead to either lying or truth telling. 
There were similar differences in trust-related explanations between help conditions. Participants who were helped and did not corroborate the alibi were more likely to report doing so because of a lack of trust in the confederate than those who were not helped and did not corroborate (39\% vs. 14\%). On the other hand, participants who were helped and corroborated the alibi were more likely to report doing so either because they trusted the confederate, or because they didn't think going to the restroom was important to report, compared to those who were not helped and corroborated (54\% vs. $17 \%$ and $30 \%$ vs. $8 \%$, respectively). It seems, then, that participants who were previously helped by the confederate were more likely to invoke trust-related explanations. It is possible that non-corroborators interpreted the nature of the confederate's help as morally-questionable (i.e., cheating) more so than did corroborators. Unfortunately, however, no measure of participants' interpretation of the confederate's help was obtained.

Whereas study 1 explored the impact of liking on false alibi corroboration, and study 2 looked at the influence of implicit increases in social pressure on false alibi corroboration, study 3 was designed to further explore the role of affinity between alibi provider and witness on corroboration rates. Although an increase in a witness' liking of a suspect, and the introduction of a reciprocal helping situation, did not impact rates of false alibi corroboration, it is still possible that a real friendship (which is likely characterized by both mutual liking and reciprocity, among other positive qualities) may sufficiently motivate an alibi witness to lie for a suspect. Thus, the next study builds on the previous two by going beyond the effects of liking and social pressure and comparing alibi witness and suspect pairs who were strangers to those who shared genuine 
relationships. The 'confederate' (i.e., suspect) in study 3 was a randomly chosen pair member who was recruited and 'trained' on the spot. 


\section{Study 3 - The effect of friendship on corroboration of a false alibi}

Under the pretext of a problem-solving study investigating the role of interpersonal closeness on performance, participants were tested in gender-matched dyads - completing the study with either a friend or a stranger. During the first part of the experimental session, the dyad completed filler tasks individually, during which time one of the two participants was recruited as a confederate. During the second part of the experimental session, dyads completed a collaborative task. During this task the newly recruited confederate momentarily left the study room under the pretext of needing to use the restroom. Following this phase the experimenter questioned the dyad about a theft that had just occurred, for which the confederate offered a false alibi. The naïve participant was later given the opportunity to either corroborate the confederate's alibi that he or she remained in the study testing room during the time of the crime, or to refute

it. The design of this study was a one-way quasi-experimental design with two levels of the independent variable (relationship condition: Friends vs. Strangers). The main dependent variable was the participant's corroboration or non-corroboration of the confederate's alibi.

\section{Participants}

One hundred and sixty participants (80 pairs) took part in the study. Participants in both relationship conditions were recruited via SONA. Three separate studies were advertised on SONA: One was for the Friends condition, for which introductory psychology students were instructed to bring a same-gender friend with them to the study session. The other two ads were for the Strangers condition (one for males and one for 
females), and were almost identical to the advertisement used for study 1 (see Appendix F for all material used for study 3). For this study, however, two participants could sign up for each session. In the event that two friends intentionally signed up for the Strangers conditions on SONA, they were re-assigned to the Friends conditions by the experimenter. All participants who signed up for the Strangers condition were introductory psychology students, whereas of those who signed up for the Friends condition, one participant per session was an introductory psychology student and the other was not (although most friends were still undergraduate students). Introductory psychology students received one course credit and non-intro psych friends received ten dollars as compensation for their participation. The age of the naïve participants ranged from 17 to 33 years with an average of 19.32 years $(S D=2.28)$.

Two participants were dropped from the data set and subsequent analyses due to their strong disbelief of the cover story, as became evident during the debriefing session. Eight more participants were dropped due to the newly recruited confederate not following the instructions (see Procedure section). One participant was not paying attention and was surprised to hear about the mock theft at the debriefing interview, and a final participant refused to wear the heart monitor. Thus, the data from a final sample of 136 participants (68 pairs, 59 of which were female pairs) were retained for analyses.

\section{Setting and Apparatus}

This study took place in the same setting as study 1 , and the same equipment was used. A concealed voice-activated Sony ICD-PX720 digital audio recorder was used to record the study session to ensure that the recruited confederates did not reveal the true purpose of the study to the naïve participants. 


\section{Measures}

Demographic and Relationship Questionnaire. Participants in both conditions completed a brief demographic questionnaire (age and gender), which also included questions about the nature and duration of their relationship with the other participant. In the Strangers condition, participants were asked to confirm that they did not know their study partner. When they did (i.e., when two friends signed up together), they were reassigned to the Friends condition.

Friendship Qualities Scale (FQS). Participants in the Friends condition completed the Friendship Qualities Scale (FQS, Bukowski, Hoza, \& Boivin, 1994) to assess the closeness of the relationship between each participant and his or her friend. This 23-item scale, which measures five dimensions of friendship (Companionship, Closeness, Help, Security, and Conflict), has been shown to have good reliability and validity with both child and adult samples (Bukowski et al., 1981; Ponti, Guarnieri, Smorti, \& Tani, 2010). Ponti et al.'s (2010) version of the scale items was used as it uses language appropriate for adult relationships (e.g., 'If other people were bothering me, my friend would help me' vs. 'If other kids were bothering me, my friend would help me').

Right-Wing Authoritarianism (RWA) scale. Participants completed the RWA (Altemeyer, 1981 - see study 2 for description), again included as an exploratory measure. It was possible that those participants who scored higher on authoritarianism would be less willing to lie to an authority figure (i.e., the experimenter) and thus less likely to corroborate a false alibi. 
Alibi Provider's Prediction. In order to be able to compare alibi providers' expectations of a witness' corroboration of a false alibi in a specific situation to the alibi witness' actual response, the alibi provider in each session was asked to predict whether or not the naïve participant would corroborate his or her alibi.

Post-Measure Questionnaire. The naïve participant answered this filler questionnaire at the end of the experimental session. It was administered in order to provide an opportunity for the dyad to be separated and for the experimenter to question the naïve participant about the recruited confederate's alibi (see Procedure section, Study 1).

Debriefing Questionnaire. Participants were asked a series of questions during the debriefing interview in order to assess their beliefs about the experimental situation (the same debriefing questionnaire used for study 2 was used for study 3 ). The experimenter asked participants whether they heard the other participant provide the false alibi, whether they were suspicious of the other participant's role as a confederate, and to what extent they suspected that the confederate was guilty of stealing the money. Participants were also asked to explain why they did or did not corroborate the confederate's alibi.

\section{Procedure}

An experimental paradigm similar to the one used in Study 1 was used. The first part of the experimental session (the 'Word generation task' and 'Liking questionnaire' phases) was modified to accommodate for the need to recruit one of the dyad members as a confederate. The second part of the experimental paradigm (the 'Restroom break', 
'Mock theft and false alibi', 'Alibi corroboration or rejection', and 'Debriefing

interview' phases) was identical to the No Request condition of study 1 , again with the exception that participants were asked about the duration of the confederate's absence and his or her direction of travel.

From the SONA study ad, participants were informed that the purpose of the study was to investigate the influence of personality and interpersonal closeness on dyadic performance on problem-solving tasks. Participants in the Strangers condition were told that they were in the 'control group' for this study (those who were not interpersonally related). Participants were tested in pairs, however one of the dyad members was recruited to act as a confederate. Upon arrival at the laboratory, dyad members were assigned to be the confederate (from here on referred to as the 'alibi provider') or the naïve participant (from here on referred to as the 'alibi witness'), based on which seat they chose in the testing room. After signing consent forms, they were instructed that the first half of the study would entail completing a set of questionnaires and a problem-solving task individually, that the second part of the study would entail completing a set of problem-solving tasks together, and finally the third part would entail completing some questionnaires individually.

Recruitment Phase. After being informed of the general study procedures, the alibi provider was asked to go in an adjacent room to complete the first part of the study individually. The alibi witness completed a questionnaire package that included the RWA scale (under pretext of needing a personality measure) and the Demographic and Relationship questionnaire. Alibi witnesses in the Friends condition were also asked to complete the FQS. Following the questionnaire package the alibi witness was asked to 
complete a filler task (the word generation task used in Study 1). During this time, the alibi provider was recruited as a confederate. He or she was informed of the true purpose of the study (i.e., that I was investigating the frequency of altruistic lying in an alibi corroboration context) and asked to sign a second consent form. He or she was then given instructions for the second part of the study: During the image-rating task (see Procedure for study 1), he or she would excuse him or herself to go to the restroom. After a 3-minute period (timed by the experimenter), he or she would return to the study room and continue with the assigned tasks. When the experimenter announces the theft and inquires about it, the alibi provider was to say: "No, we were both in here the whole time". The experimenter stressed the importance of not telling the alibi witness about the true nature of the study, and informed the alibi provider of the concealed audio-recorder in the study room (and consent for this audio-recording was also obtained at this point). The experimenter also asked the alibi provider to re-iterate what they were asked to do to ensure they understood the instructions. Finally, the experimenter asked the alibi provider to predict whether the alibi witness would lie for them by corroborating their false alibi, or tell the truth and report their absence.

The Restroom break, Mock theft and false alibi, Alibi corroboration or rejection, and Debriefing interview phases proceeded exactly as they did in Study 1 (NR condition). Alibi providers were debriefed while alibi witnesses answered the filler post-measure questionnaire, and the latter were debriefed separately immediately after, during which time the experimenter administered the debriefing questionnaire. A written consent for the use of the audio recording was also obtained. Again, no participant expressed concerns over the deception used, and most stated that they enjoyed the study. 


\section{Results}

\section{Sample Description: Strangers and Friends}

Thirty-four pairs of strangers ( 7 of which were males) and 34 pairs of friends ( 2 of which were males) participated in this study. The alibi witnesses in the Strangers condition were between 18 and 33 years old, with a mean age of $19.82(S D=2.80)$. The alibi witnesses in the Friends condition were between 17 and 23 years old, with a mean age of $18.82(S D=1.49)$. Most participants in the Friends condition brought with them someone they described as a friend (31 out of 34), two participants brought a family member (a sister and an aunt), and one brought a romantic partner. These individuals had known each other for an average of 5.46 years $(S D=5.73)$, ranging from less than one year to 21 years. Most (61.8\%) had known each other for more than 3 years, $8.8 \%$ had known each other between 1 and 3 years, 14.7\% had known each other between 6 and 12 months, and $14.7 \%$ had known each other for less then 6 months at the time of the study. They reported seeing each other on average 11.04 times per month $(S D=8.64)$, ranging from less than once a month to 30 times (i.e., every day). Alibi witnesses in the Friends condition also completed the Friendship Qualities Scale (FQS). Their average score on the scale was $85.53(S D=11.37$ ), and ranged from 48 to 100 (out of a maximum score of 110).

Attention to Alibi. Predictably, a substantial subset of alibi witnesses (36.8\%) reported during the debriefing interview that they did not hear or did not remember hearing the confederate state the alibi. This may have been more likely to have genuinely happened in this study because less control could be exerted over how the confederates, recruited on the spot, expressed the alibi. Thus, again as a precaution, all main analyses 
were computed with and without these cases, to ensure alibi witnesses did not respond differently based on whether or not they heard the alibi.

\section{Main Analyses}

Twenty out of 68 alibi witnesses (29.4\%) corroborated the confederate's false alibi by confirming that he or she was in the study room during the time the theft occurred. Of the participants who instead reported to the experimenter that the confederate had left the room, the majority (46 out of 48) did so when the experimenter asked them to confirm the alibi. The remaining two participants reported the confederate's absence by 'reminding' the latter that they went to the restroom immediately when they stated the false alibi.

A chi-square test of independence between relationship condition and alibi witnesses' decision to corroborate or reject the alibi revealed a significant association, $\chi^{2}(1)=4.53, p=.03, \phi=.26$. As expected, alibi witnesses in the Friends condition were more likely to corroborate the false alibi than those in the Strangers condition $(41.2 \%$ vs. 17.6\%, Table 3). The odds that an alibi witness corroborated the false alibi were 3.27 times higher if the confederate was a friend than when the confederate was a stranger.

To ensure this difference held among participants who reported that they did not hear the confederate's alibi, the chi-square test was repeated without these participants. The effect was near significant, and was just as strong, $p=.08, F E T, \phi=.31, O R=5.10$. Similarly, when alibi witnesses who reported that they corroborated the false alibi because they forgot that the confederate had gone to the washroom were omitted, the effect remained, $p=.01, F E T, \phi=.34, O R=11.20$. 
Table 3.

Alibi Corroboration Contingency Table

\begin{tabular}{l|cc|cc|cc}
\multicolumn{2}{c|}{} & \multicolumn{2}{c|}{$\begin{array}{c}\text { Participant did not } \\
\text { corroborate alibi }\end{array}$} & \multicolumn{2}{c|}{$\begin{array}{c}\text { Participant } \\
\text { corroborated alibi }\end{array}$} & \multicolumn{2}{|c}{ Total } \\
& $N$ & $\%$ & $N$ & $\%$ & $N$ & $\%$ \\
Strangers & 28 & 82.4 & 6 & 17.6 & 34 & 100 \\
\hline Friends/Related & 20 & 58.8 & 14 & 41.2 & 34 & 100 \\
\hline Total & 48 & 70.6 & 20 & 29.4 & 68 & 100
\end{tabular}

To investigate whether certain characteristics of the friendship between alibi witnesses and confederates were associated with corroboration decision, FQS scores, how long each pair had known each other for (in years), and how often they saw one another (per month) were entered in a logistic regression analysis. None of these variables, however, predicted the likelihood of corroboration (FQS scores: $\beta=0.001(S E=0.04)$, $p=.98, O R=1.00, \mathrm{CI}_{.95}[.93,1.08]$; relationship duration: $\beta=-0.02(S E=0.11), p=.87$, $O R=0.98, \mathrm{CI} .95[.80,1.21]$; frequency: $\beta=-0.05(S E=0.06), p=.42, O R=0.95, \mathrm{CI} .95$ $[.84,1.08]) .{ }^{11}$ Thus, relationship closeness could not further predict alibi corroboration within the Friends condition.

Suspects' Prediction of Alibi Witnesses' Corroboration Decision. Before the mock theft phase of the study, newly-recruited confederates were asked to predict the other participant's response when they would be asked to confirm the false alibi. To examine the confederates' success in their prediction, in both relationship conditions, a

\footnotetext{
${ }^{11}$ Again, similar non-significant results were obtained when excluding participants who reported not remembering that the confederate had left the room.
} 
three-way hierarchical loglinear analysis including relationship condition, alibi corroboration prediction, and alibi corroboration outcome was computed. The final model retained the relationship condition $\times$ alibi corroboration outcome interaction (reiterating the findings described above), as well as the relationship condition $\times$ alibi corroboration prediction interaction. The likelihood ratio for this model was $\chi^{2}(2)=2.13$, $p=.34$. Confederates in the Friends condition were more likely to predict that the alibi witness would corroborate their false alibi than confederates in the Strangers conditions $(44.1 \%$ vs. $14.7 \%), \chi^{2}(1)=7.33, p=.007, O R=4.58$. Friends' predictions, however, were neither more or less accurate than strangers' predictions, $p=.19$. In fact, confederates' predictions overall were not associated with actual corroboration outcome, $p=.51$ (Figure 4). The accuracy rate of confederates in the Friends condition was 50\%, and the accuracy rate of confederates in the Strangers condition was $68 \%$.

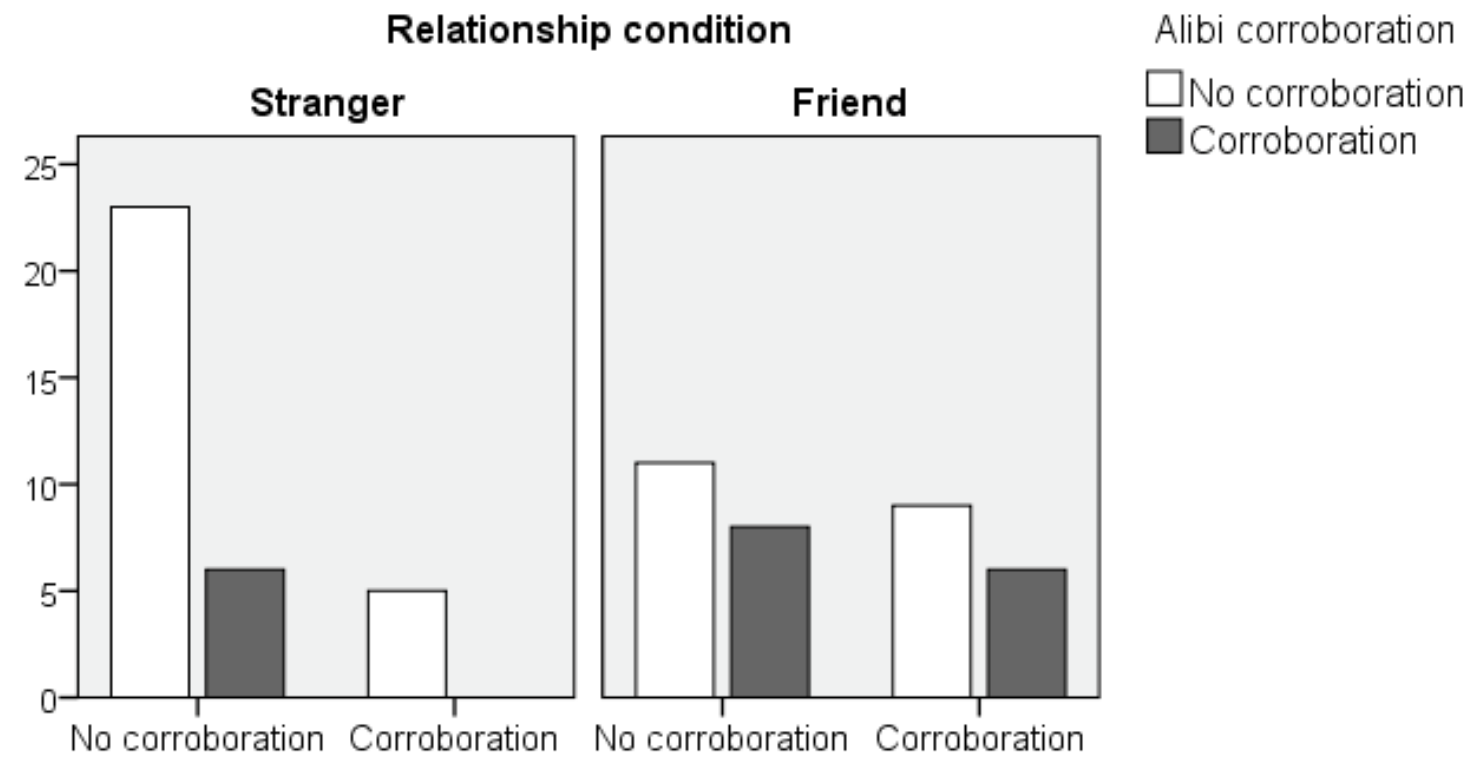

Predicted corroboration

Figure 4. Number of alibi witnesses who corroborated the false alibi, as a function of relationship condition and confederates' predictions 


\section{Exploratory Analyses}

Estimates of Absence Duration and Direction of Travel. Alibi witnesses who did not corroborate the confederate's alibi were asked to estimate the duration of the confederate's absence, and if they knew which way the confederate turned when he or she exited the room. These questions were used to assess if alibi witnesses would provide information suggesting either the guilt or innocence of the confederate. Since alibi witnesses in the Strangers condition reported being more suspicious of the confederate's guilt $(M=3.79, S D=2.53$, on a 10 -point Likert scale) than alibi witnesses in the Friends condition $(M=1.49, S D=1.10, t(43.53)=4.81, p<.001, d=1.19)$, it was possible that the former may have provided more incriminating answers than the latter. Again, however, many alibi witnesses did not provide answers to these questions. Twenty-seven (out of 48) did not provide a time estimate for the confederate's absence. Of those who did, the average reported duration was 2.36 minutes $(S D=0.81)$ and ranged from 1 to 4.5 minutes. A $t$-test revealed no differences in duration estimates between alibi witnesses in the two relationship conditions, $p=.36, d=0.41$. Duration estimates, however, were moderately and positively correlated with alibi witnesses' suspiciousness ratings, $r_{s}=.37$, although this did not reach significance, $p=.095$. Most alibi witnesses (40 out of 48) did not provide a direction of travel. Of those who did, 4 (3 from the Friends condition) stated that the confederate turned left, toward the office, and 4 (3 from the Friends condition), stated that the confederate turned right, toward the restrooms. No comparison between groups was possible due to the low response rate of this question.

Exploring Predictors of False Alibi Corroboration. The FQS measures five dimensions of friendship (Companionship, Closeness, Help, Security, and Conflict). It 
was possible that strength in certain aspects of friendship, for example feeling a responsibility to help the other (Help dimension, Ponti et al., 2010), would increase the likelihood of alibi corroboration, whereas strength in other aspects, for example feeling that a friendship is secure (Security dimension, Ponti et al., 2010), would decrease the likelihood of corroboration. However, due to the small sample size (27 in the Friends condition who completed the $\mathrm{FQS}^{12}$ ), not all five dimensions were explored as potential predictors of corroboration decisions. Thus, the two dimensions described above, Help and Security, along with Closeness (a measure of the emotional bond between friends), were entered in an exploratory stepwise (backward) logistic regression analysis to assess their predictive ability. The two other dimensions, Companionship (a measure of how much time friends spend together) and Conflict (a measure of the frequency of disagreements) were excluded based on an assumption that these concepts would be less relevant to altruistic lying. None of the predictors, however, were retained in the model, all $p \mathrm{~s}>.54$, model $\chi^{2}(1)=0.18, p=.67$.

Finally, the RWA scores and suspiciousness ratings of all alibi witnesses (i.e., in both conditions) were again investigated as potential predictors of alibi corroboration decision ${ }^{13}$. A combined hierarchical and stepwise logistic regression analysis was conducted to see if either of these two variables could significantly predict corroboration decision beyond relationship condition (entered in the first block). The final model, however, did not retain any additional variables, $\beta_{\text {relationship condition }}=-1.15(S E=0.57)$,

\footnotetext{
${ }^{12}$ Seven alibi witnesses in the Friends condition failed to respond to half of the items on the FQS, which were printed on the back side of the page.

${ }^{13}$ Again, due to a restricted age range (66.2\% of alibi witnesses were either 18 or 19 years old), age was not investigated as a viable predictor.
} 
$p=.04$, OR $=0.32, \mathrm{CI}_{.95}[.10, .97]$, all other $p \mathrm{~s}>.13$, model $\chi^{2}(1, N=67)=4.32, p=.04$, $R^{2}=.05$ (Hosmer \& Lemeshow), .06 (Cox \& Snell), .09 (Nagelkerke). Residual and collinearity statistics were all adequate.

Reasons for Corroborating or Rejecting the False Alibi. During the debriefing interview alibi witnesses were again asked to explain why they did or did not corroborate the confederate's alibi. Appendix G shows a complete breakdown of the reasons reported by alibi witnesses in both relationship conditions. The same categories used in studies 1 and 2 were coded (see Appendix B for the category descriptions). ${ }^{14}$

The most commonly reported reasons for not corroborating the confederate's false alibi included doing so for moral reasons (30 out of 48 alibi witness, or $62.5 \%$ ), because they trusted that the confederate was innocent $(15$, or $31.3 \%)$, to avoid a confrontation or involvement in the situation (12, or $25.0 \%)$, because they did not know or trust the confederate (10, or $20.8 \%$ ), and because they were simply complying by answering the experimenter's question (8, or $16.7 \%)$. More alibi witnesses in the Friends than in the Strangers condition reported not corroborating the false alibi because they trusted that the confederate was innocent (55.0\% vs. $14.3 \%)$, whereas more alibi witnesses in the Strangers than in the Friends condition reported not corroborating because they doubted the confederate's innocence $(28.6 \%$ vs. $10.0 \%)$.

Among alibi witnesses who corroborated the alibi, the most commonly reported reason for doing so was because they believed the confederate was innocent (16 out of 20 , or $80.0 \%)$, because they forgot that the confederate had left the room (10, or $50.0 \%)$,

\footnotetext{
${ }^{14}$ Due to low cell frequencies and non-independence of data, only descriptive statistics are reported.
} 
to protect the confederate or their relationship with the confederate $(4$, or $20 \%)$, and because they were caught off guard and didn't have time to think about the situation (2, or $10.0 \%)$. One alibi witness reported corroborating the alibi to avoid confrontation or involvement in the situation, and one more stated doing so because she thought her friend was guilty.

\section{Discussion}

\section{Relationship and Alibi Corroboration}

Hypothesis 5 was supported by the data: Alibi witnesses were more than twice as likely to corroborate a friend's false alibi as they were to corroborate a stranger's false alibi. Among friends, however, the strength or length of the relationship did not appear to be associated with alibi corroboration decision. Still, this finding lends some validity to the assumption that is consistently found in the psychological literature on alibis that friends are more likely to lie for one another than are strangers (e.g., Olson \& Wells, 2004; Hosch et al., 2010). Not surprisingly, alibi witnesses were more suspicious of the confederate's guilt when the latter was a stranger than when he or she was a friend. Alibi witnesses in the Friends condition overwhelmingly believed that their friend was not involved in the theft, and according to these individuals' self-reports (see section below), this trust seemed to have influenced their decisions of whether or not to corroborate the false alibi. Despite this difference, however, both groups, on average, were equally accurate at reporting the events that occurred when they did not corroborate the false alibi (i.e., the duration of the confederate's absence and his or her direction of travel). Participants' suspiciousness of the confederate's guilt, on the other hand, was correlated with reported time estimates. Higher beliefs in the suspect's guilt were associated with 
longer estimates of the duration of the confederate's absence. Interestingly, it was generally those alibi witnesses who gave the lowest suspiciousness ratings (1 out of 10) who underestimated the duration of the absence, rather than those who were more suspicious giving overestimates. It is unclear, however, whether more or less suspiciousness led to a cognitive bias influencing their estimates of time, or whether this correlation reflected a more conscious effort to direct blame away from the confederate.

\section{Suspects' Predictions of Alibi Corroboration}

An interesting finding from this study comes from confederates' predictions of whether the alibi witness (i.e., the other participant) would lie for them or tell the truth. Suspects in both relationship conditions were not very accurate in their predictions. Friends of alibi witnesses performed at chance level (50\%), whereas strangers were above chance (67\%), but still not significantly more accurate than friends. In fact, the increase in accuracy in the Strangers condition was due to the overwhelming prediction of non-corroboration (i.e., a prediction that alibi witnesses would tell the truth), a prediction somewhat akin to the truth bias often found in lie detection studies (e.g., Culhane et al., 2013).

The findings that confederates in the Friends condition were almost three times more likely than those in the Strangers condition to predict that the alibi witness would corroborate their false alibi is in line with previous self-report research showing that mock suspects often report that they would rely mostly on friends or family members to corroborate a false alibi (vs. strangers, e.g., Hosch et al., 2011). However, findings from this study would suggest that the friends that are relied upon may not be the same ones who would actually lie for them, or at least not based on an implicit request to lie as was 
the case in this study (i.e., it is possible that individuals' predictions about which of their friends would lie for them upon directly asking them to may be more accurate). Although studies that have asked mock suspect to collect corroborating evidence for a false alibi have found that friends are more likely to provide such corroboration than strangers (who almost never do, e.g., Culhane et al., 2013), it is difficult to determine with certainty whether these corroborators were the same individuals initially relied upon by the 'suspect', and whether these friends would actually lie in a real-life setting.

\section{Reasons for Lying and Telling the Truth}

Two interesting patterns from alibi witnesses' explanations of why they did or did not corroborate the confederate's false alibi that were found in the first two studies were observed again in this study. First, alibi witnesses reported that their trust in the confederate's innocence was a reason for both lying and telling the truth. However proportionally more alibi corroborators invoked trust in the confederate as a reason for their decision than non-corroborators (80\% vs. $31 \%)$. Moreover, in this study, alibi witnesses in the Friends condition were more likely than those in the Strangers condition to state a trust in the confederate as their reason for their decision, regardless of whether they did or did not corroborate the false alibi (68\% vs. 24\%). Conversely, alibi witnesses who did not corroborate the alibi were more likely to report doing so because they did not know or trust the confederate if the confederate was a stranger than if he or she was a friend (29\% vs. $10 \%)$. Thus, overall, it seems alibi witnesses were more trusting of their friends than they were of strangers.

Second, as in studies 1 and 2, many of the alibi witnesses in this study who corroborated the false alibi stated that they failed to report the confederate's absence 
because they had forgotten, at the time the experimenter questioned them, that confederate had left. But again, although some may have genuinely forgot, others may have provided that answer as a way to explain their behaviour as socially acceptable (i.e., not as a lie). Evidence supporting this idea comes from the high number of 'forgetters' who also stated other reasons for corroborating the alibi, in addition to their memory lapse (usually that they also trusted the confederate was innocent). A portion of these participants, however, also stated that they remembered the confederate's absence a few minutes after corroborating the alibi and consciously decided not to change their corroboration. Thus, whether reports of initial forgetting were always genuine or not is difficult to determine, but at least some of these alibi witnesses nevertheless genuinely corroborated the alibi in the end.

This study has shown that this experimental paradigm can be successfully used with pairs of participants with pre-existing relationships, in which one person can be rapidly recruited as a confederate. In this study, friends were recruited primarily because of convenience: It was more likely that a student would have a friend readily accessible on campus, rather than a family member, romantic partner, or co-worker. However this paradigm could easily be used, and should be used in future research, with pairs of participants who share different types of social and familial relationships. It would be interesting to compare the rates of actual false alibi corroboration with the rates of hypothetical willingness to lie in an alibi corroboration context reported in Hosch et al. (2011), for all levels of relatedness. As was done in the previous studies, other factors could also be manipulated, such as increased social pressure to lie via explicit requests, and the suspiciousness of, or level of evidence against, the alibi provider. We found in 
Marion and Burke (2013) that increasing the suspiciousness of a suspect reduces the rate of false alibi corroboration among stranger/co-worker alibi witnesses, however it is unclear whether such a drop in altruistic lying would be found among friends or family members, given how trusting friends were of one another in the current study. It is possible that what a stranger would interpret as suspicious behaviour (e.g., a confederate handling money around the time of the theft) would not be appraised as such by a mother who would never think of her son as a thief, for example. In other words, the point at which contextual information increases suspiciousness of a suspect may be higher for close others. The paradigm described herein could easily be adapted to answer these questions. 


\section{General Discussion}

\section{False Alibi Corroboration}

The goal of the research described above was to identify factors that can influence the likelihood that a witness will lie for a suspect by corroborating a false alibi, or in other words, factors that can predict the reliability of alibi witnesses. A novel experimental design permitted the direct observation of alibi corroboration behaviour in a setting that was both reasonably ecologically valid and ethically sound. The factors examined in the three studies conducted can be classified into three groups: characteristics of the relationship between a suspect and an alibi witness, elements that create social pressure, and individual differences.

Findings from studies 1 and 2 suggest that an increase in liking of an individual does not increase the likelihood that one will lie for that person in an alibi corroboration context. The liking manipulation in study 1 was a direct replication of that used in Marion and Burke (2013), who also did not find an association between false alibi and liking, despite a successful manipulation of how much alibi witnesses liked suspects. The original finding and its replication here comes as a bit of a surprise given the evidence from the social psychological literature that increased liking and perceptions of similarity (which were incorporated in the liking manipulation) often increases compliance rates (e.g., Burger et al., 2001; 2004; Cialdini \& Trost, 1998). Additional evidence that an increase in liking of a suspect does not lead to a higher likelihood of false alibi corroboration also comes from results obtained in study 2. Although liking was not directly manipulated, it seemed to have been an indirect effect of the helping manipulation. Alibi witnesses who had been previously helped by a confederate liked 
him or her more than those who were not previously helped, yet the alibi corroboration rate in the two groups was equal. A possible explanation for this lack of effect is that liking increases compliance only to explicit requests, as opposed to the implicit request made by confederates in these studies who simply stated a false alibi in the participants' presence. However, liking did not increase the rates of corroboration even when an explicit request to do so was made by the confederate in study 2. Another alternative, then, is that a relationship between liking and compliance does not apply to situations in which the act being requested is morally questionable, or in situations in which the request (either implicit or explicit) is not taken seriously. Support for these ideas comes from participants' self-reported explanations for why they did not corroborate the confederate's alibi. A large proportion of participants in studies 1 and 2 reported that they told the experimenter about the confederate's absence because of moral reasons (e.g., 'lying is wrong' or 'thefts are serious', 79-83\%), and a few more reported doing so because they did not perceive the absence as anything of importance that needed to be concealed (and thus undermined the confederate's wish for them to lie, 2-20\%).

Although the level of affinity toward a suspect did not affect the likelihood that a witness would corroborate his or her false alibi, the type of social relationship shared between a suspect and a witness did have such an effect. Witnesses who were friends of suspects in study 3 were over twice as likely to lie for him or her as witnesses who did not know the suspect (i.e., strangers). This finding is in line with the extant literature on alibi evidence describing investigators' and jurors' scepticism of alibis that are corroborated by close others, seemingly due to the assumption that these individuals are motivated to lie for one another whereas strangers are not (e.g., Dysart \& Strange, 2012; 
Olson \& Wells, 2004). Lying for a suspect by corroborating a false alibi has been described as an altruistic act due to the potential risks to the corroborator that accompany the benefits to the suspect (Hosch et al., 2011; Marion \& Burke, 2013). Thus, the effect of relationship type on corroboration rates found in study 3 also supports evolutionary psychology's reciprocal altruism theory (Trivers, 1971) which states that socially-related others are more likely to act altruistically toward one another than strangers not socially related. However, this same theory would suggest that the rate of altruistic behaviour found among socially-related others would increase with the closeness of the relationship, which was not found in study 3. It is quite likely, however, that due to the dichotomous nature of the outcome measure and the limited range of social relationship (i.e., $91 \%$ of pairs in the Friends condition were friends, as opposed to co-workers, neighbours, or other social relationships), this study was underpowered to uncover such finer associations.

There were some interesting differences between the rate of corroboration observed in the behavioural paradigms used in the current studies and the expected and predicted rates of corroboration described in studies that have used self-report measures. The overall rate of corroboration among friends in study 3 was $41 \%$, whereas the overall rate of corroboration among strangers in studies 1 (NR condition only), 2, and 3 was between $18 \%$ and $31 \%$. When participants in Hosch et al.'s (2011) study were asked to estimate the percentage of alibi witnesses who would lie in court to protect an armed robbery suspect, their estimate was around $6 \%$ for strangers, $15 \%$ for familiar strangers $31 \%$ for co-workers, and $78 \%$ for best friends. Thus, it would appear that estimates of lying among friends may be too high, and that estimates for lying among strangers are 
much too low. If, however, 'strangers' in the current studies can be more aptly categorized as familiar strangers or even coworkers (or in this case students attending the same school), then predictions of how much these individuals lie are more accurate. Of course this is assuming that these predictions are based on situations in which the suspect makes no direct request to the alibi witness to lie. If these estimates are assuming that direct social pressure from a suspect was applied to the alibi witness (as is likely in actual cases), then in comparison to the false alibi corroboration rates obtained among strangers in study $1(61 \%)$, expected lying rates among strangers are grossly underestimated. An alternative explanation for the discrepancy between the rates of alibi corroboration obtained in the current studies and those obtained by self-report measures in Hosch et al. (2011) may lie in the lack of contextual information provided to participants in the latter. It is possible that self-report studies that include a more complete picture of the details surrounding the type of crime, level of evidence against a suspect, and the nature of the interaction between a suspect and a witness (e.g., implicit or explicit request to lie), may obtain figures that better mirror those obtained by behavioural measures, and future research should address this possibility.

Adding direct social pressure to lie, in the form of a request to withhold information about a suspect's true whereabouts, more than doubled the rate of false alibi corroboration (study 1). Indirect social pressure to return a favour through the norm of reciprocity, however, did not have an effect on participants' decisions to lie or tell the truth (although as previously explained, the help manipulation used in this study may not have been salient enough to produce an effect). It is also important to note that the situation created in this paradigm made the guilt of the confederate ambiguous. The 
confederate did not ask participants to lie about him or her stealing, or even about being in the vicinity of the crime scene. Instead, the confederate asked the participant not to reveal that (s)he went to the washroom for fear that the experimenter would disapprove of him or her leaving the during the study session. It was quite likely that fellow students had empathized with this concern and thus were motivated to lie to protect the confederate from an unrelated 'transgression' and not because they thought he or she was guilty. This was reflected in participants' reports of why they corroborated the alibi: More participants in the request condition (57\%) than in the no request condition $(30 \%)$ said that they lied to protect the confederate. It is quite possible, however, that an alibi witness' knowledge of the suspect's guilt would yield different results (e.g., a decrease in the rate of false alibi corroboration).

The personality characteristics of the alibi witnesses in the current studies did not seem to be associated with altruistic lying in an alibi corroboration context, however gender may be a factor worthy of further investigation in future research. Although men in study 2 were somewhat more likely to lie for a male confederate than women were to lie for a female confederate, an obvious confound in the design was the gender of the confederate. Because pairs were gender-matched, it is impossible to tell if the effect observed was because male alibi witnesses are more likely to lie, or because people in general are more likely to lie for male suspects. But because the psychological literature on lying reports that other-oriented lies (which encompass altruistic lies) are more likely to be told to benefit women than men (e.g., DePaulo et al., 1996), and because most individuals are likely to stereotype criminals as being male (e.g., MacLin \& Herrera, 
2006), which in turn should make male suspects more suspicious, I believe that the former explanation is the most likely.

Although it is sometimes difficult to compare results between studies, the differences and similarities in corroboration rates obtained in these three studies deserve a mention. Since the experimenter was the same person, and the procedures and participant pool very similar in all three studies, it is reasonable to assume that any major differences in corroboration rates can be attributed to the independent variables investigated. The corroboration rate among female, non-friend pairs is fairly consistent, varying between $18 \%$ (strangers in study 3 ) and $26 \%$ (NR condition in study 1). These values are also consistent with the rate of corroboration obtained by Marion and Burke $(23 \%, 2013)$. Increases in corroboration rates were obtained in study 1 when a request to lie was added $(61 \%)$, when men were recruited (41\%), and when friends were recruited (41\%). This observation lends further support to the conclusions that the request manipulation (study 1), participant gender (study 2), and suspect-witness relationship (study 3) had true effects on the corroboration rates of alibi witnesses. Future research should explore whether a combination of these factors would have a cumulative effect on the corroboration rate of alibi witnesses. For example, it is possible that a suspect who asks a friend to withhold information about their whereabouts may be even more likely to lie for them than strangers who are asked the same or friends who are not asked to withhold information. If, however, a request to withhold information simply engenders, in a stranger, sympathy towards a suspect whom they believe is innocent, a request may not further increase an already-sympathetic friend's motivation to lie for him or her. 


\section{Memory for Alibi Events}

Given the significant number of participants in all three studies who reported corroborating the false alibi due to forgetting the confederate's absence, it is debatable whether a good proportion of the false alibi corroborations provided by participants can even be classified as lies. Just as suspects often do not always have a great memory of where they were and what they were doing at the time of a crime (e.g., Olson \& Wells, 2010), it is not inconceivable that alibi witnesses would have a less than perfect memory for the whereabouts and actions of another person. Of course what is surprising in these claims of failed memory in these cases is the very short period of elapsed time between the event itself and the misreport of the recalled event. From the time the confederate returned to the study room until the time the experimenter asked the participant to confirm the alibi, 10 minutes, at most, would have elapsed. Most investigators, it seems, would be sceptical of such memory errors. In Dysart and Strange's (2012) survey, 93\% of police officers reported that an honest error in an alibi would be extremely unlikely to occur after 10 minutes of an event in question. When an error does happen within such a short timeframe, investigators most commonly attribute these errors to the influence of drugs or alcohol, mental illness, or intense trauma or stress. This combined with a general mistrust of changed alibi stories (regardless of whether the change weakens or strengthens the alibi, e.g., Culhane \& Hosch, 2012) does not fare well for suspects whose alibi witnesses are inconsistent in their accounts of relatively recent events. Unfortunately, however, as was consistently found in all three studies herein, memory errors for other people's recent activities do seem to occur in the absence of, as far as I could tell, drugs, mental illness, or severe stress. A more reasonable explanation for 
participants' forgetfulness may be something akin to inattentional blindness (Mack \& Rock, 1998), where participants' attentional focus on the filler task during the confederate's absence made them functionally blind to the latter's exit and return.

However, no participant ever claimed to not remember the confederate leaving once they were reminded by the experimenter, suggesting that there was not a complete lack of encoding of the event (Simons, 2000). Thus, what an alibi witness is doing during the time covered by an alibi may impact the reliability of his or her account of the suspect's activities.

\section{Motivations to Lie: How Trust Can Backfire}

It was first reported in Marion and Burke (2013) that alibi witnesses often lie not to cover up a bad behaviour, but to divert blame from a suspect whom they believe is innocent. The same pattern of results was found again in all three current studies. Both participants who reported the confederate's absence and those who corroborated the false alibi reported doing so because they trusted that the confederate was innocent, but in all three studies belief in the suspect's innocence led to lying more often than it led to telling the truth $(13-32 \%$ vs. $33-80 \%)$. Another related and often-stated motivation for corroborating the false alibi was because alibi witnesses believed that the confederate's actions (i.e., going to the washroom) were innocuous and irrelevant to the situation (although the experimenter clearly asked if the confederate was in the room "the whole time"). This response also implies that participants believed that the confederate had indeed gone to the washroom. Lying due to a belief in innocence, however, may be a dangerous game to play, as we know that alibis that are changed lead to the perception of a guilty alibi provider (e.g., Culhane \& Hosch, 2012), and this could possibly extend to 
the perception of a complicit alibi corroborator. Thus, if caught in a lie, even if the lie was told to divert blame away from an innocent suspect, corroboration of a false alibi could very well backfire by increasing perceptions of guilt.

\section{False Alibi Corroboration: Not Always an Altruistic Act}

The corroboration of a false alibi has often been described as an altruistic behaviour (e.g., Hosch et al., 2011; Marion \& Burke, 2013), and in fact on the surface it does contain all the elements necessary to be construed as such (i.e., an act that benefits another person, and does not benefit but instead caries significant risk to the actor). Corroborating a false alibi could potentially save another person from being sent to prison, but if caught could send the corroborator (and the suspect) to prison. The reasons provided by participants in all three studies for why they did or did not corroborate the false alibi provided a preliminary look into the motivations that people may have to lie for the benefit of a third party (i.e., to engage in true altruistic lying, as defined in the introduction). These responses do show that altruistic reasons were frequently at the heart of the corroboration (e.g., participants corroborating to protect the confederate, to comply with his or her request, because they trusted him or her and didn't want to make them seem suspicious, etc). However, there were a number of other motivations that were not geared toward benefitting the confederate but instead were selfish in nature. For example, participants frequently reported lying to avoid a confrontation with the confederate, or avoid being involved in the situation in general. Thus, although rates of altruistic lying between friends and strangers support Triver's theory of reciprocal altruism (study 3), the reported reasons for lying suggest that the decision to corroborate a false alibi or not is based on a more complicated interaction between social 
relationships, cognitive biases, and selfish motivations. Future research on alibi corroboration should attempt to investigate further the combined influence of these factors on lying in legal and criminal contexts.

\section{Limitations and Future Directions}

As is the case with any laboratory experiment, there are certain limits to the generalizability of these studies' findings. It is likely that the severity of the crime was perceived by participants to be quite low in comparison to the subject of most actual crime investigations, and similarly that the perceived consequences of being caught lying were lower than those perceived by actual alibi witnesses. One way to address this limitation would be to manipulate the type of crime (e.g., violent, non-violent, and property crime), and to better mimic a real crime scenario, for example by incorporating a mock security guard or police officer. In the current studies, the 'interrogator' (i.e., the experimenter), was also the victim of the theft, which could have shifted any motivation to help away from the confederate and toward the experimenter. Including a separate authority figure as the interrogator would increase the external validity of the experiment.

As a matter of convenience, undergraduate students were recruited to participate in these studies. However the average undergraduate student likely is not representative of the average criminal suspect or the average witness interviewed in criminal investigations. Thus, community samples, or samples from populations that likely have a higher proportion of criminals (e.g., street youth), may behave differently. Similarly, there was not enough variability in the ages of our participants to evaluate the effect of this variable. As lying does tend to decrease with age (e.g., Serota et al., 2010), it is 
possible that older alibi witnesses are less likely to lie. Future studies using behavioural measures of false alibi corroboration should attempt to increase the demographic variability of their participants.

As previously alluded to, a witness' knowledge of the guilt of the suspect may have altered the obtained rates of false alibi corroboration, as well as the influence of the several independent variables investigated. It is likely that at least a portion of actual alibi witnesses who lie to police do so under an explicit knowledge of the suspect's guilt. However, the findings obtained in studies described herein cannot be generalized to this specific situation, as the guilt of the confederate in these studies was purposefully made ambiguous. Therefore, in order to better understand the behaviours and motivations of the full range of criminal witnesses involved in investigations, a manipulation of the witness' knowledge of whether or not the suspect is guilty would be beneficial.

Finally, another interesting avenue for future research would be an attempt at determining why a witness' trust in a suspect sometimes leads to false alibi corroboration, and when it sometimes leads to reporting the truth. In all three studies conducted here, a trust in the confederate's innocence was provided as a reason for both lying and reporting the truth. For example, perhaps a deeper investigation of witnesses' appraisal of the seriousness and urgency of the situation, or their attributions of characteristics to the suspect (trustworthy, intimidating, etc.) would uncover a mediating factor between trust and false alibi corroboration. 


\section{Conclusion}

In conclusion, whereas most of the psychological literature on alibis has emphasized or hinted at a problematic mistrust of alibi witnesses who are close to a suspect (which is also evident in cases of wrongful convictions), the high rates of false alibi corroboration obtained in these studies suggest that a general trust in alibi witnesses who are strangers or distantly-related to the suspect should also be a concern. Although a friend of a suspect is more likely to lie for him or her than a stranger, the chances that alibi witnesses will corroborate a false alibi may also depend on whether they were asked to lie or conceal the truth, and whether or not they believe that the suspect is implicated in the crime. As of yet, no personal characteristics have been found to reliably predict altruistic lying in an alibi corroboration context, although witness gender may be a topic worthy of future research. This series of studies also highlights the need for more behavioural measures of false alibi corroboration, as our reflections of how we and others would hypothetically react in a certain situation is not always aligned with how we actually behave, and there are often factors influencing our behaviour that are unknown to us. 


\title{
APPENDIX A: MATERIAL FOR STUDY 1
}

\author{
Recruitment Advertisement for Study 1
}

\section{Study Name: Does Personality Affect Group Performance? MALES/FEMALES ONLY}

\section{Abstract:}

This 60-min social/cognitive study examines the role of personality traits and personality similarity or dissimilarity between peers on problem-solving. Participants will complete individual and group tasks and answer personality questionnaires.

\section{Description:}

The study is available to male students and will take place at the South Bond Building (105 Bond Street), 2nd floor. Please call ext. 2190 upon your arrival and wait at the main lobby area on the ground floor (do not go up the stairs but please instead use the phone near the elevator on the ground floor). All participants will be students at Ryerson University who are currently enrolled in an introductory psychology course. The session will be conducted with two participants at a time. Participants will complete a word generation task, a world-scramble task, and a memory task. They will also answer questions about their problem-solving strategies, their personality, and their opinions on the tasks. Some of the tasks will be done individually and some you will be doing collaboratively with the other participant. Part of the study session will be audio-recorded, and you may be asked to provide a physiological measure. The results of this study will help us better understand human behaviour and attitudes. Minimal anticipated risks are involved. It is possible that you may feel slight discomfort in answering some questions relating to beliefs or personal opinions. Participating in a study may be a new experience for you, and you can stop your participation either temporarily or permanently at any point without penalty. You are also free not to answer any and all questions you feel uncomfortable answering without penalty. Please arrive no earlier than 5 minutes before your scheduled session. 
Consent Agreement

Does Personality Affect Group Problem-Solving?

You are being asked to participate in a research study. Before signing this consent form, it is important that you read the following information. You may ask as many questions as necessary to be sure that you understand what the study entails.

Investigators:

\begin{tabular}{|c|c|}
\hline Stephanie Marion, B.Sc., M.A. & Tara Burke, Ph.D. \\
Graduate Student \\
Ryerson University & Department of Psychology \\
& Ryerson University \\
\hline
\end{tabular}

Purpose of the Study: The primary purpose of this study is to examine how individuals with similar or dissimilar personality characteristics work together toward a common goal. We are hoping to recruit 78 students to participate in this research. All students taking Introductory Psychology (Psy102 or Psy202) are eligible to participate.

Description of the Study: If you decide to participate in the research, you will be asked to do the following: you will complete a word-generation task, a word scramble task and a memory task. Some of these tasks you will do individually and some you will be doing with another participant with whom you have been matched based on similar or dissimilar personality characteristics. Your responses to part of this study will require audio-recording. You may also be asked to provide a physiological measure during part of this study. This would entail wearing a pulse monitor on your index finger to provide a physiological measure in response to images. This study will take place in psychology lab at 105 Bond St and will take approximately 60 minutes to complete.

What is Experimental in this Study: None of the procedures or questionnaires used in this study are experimental in nature, in the sense that they have all been used in previous cognitive and personality research. From a technical or procedural point of view, part of this study is considered "experimental", because by following the procedure described above, the study examines the impact of one variable (called the "independent variable") on another variables (called the "dependent variable"). More information about the independent and dependent variable will be provided at the end of the session.

Risks or Discomforts: Occasionally people feel uncomfortable when answering questionnaires that ask about attitudes and personal opinions toward controversial social issues (e.g., affirmative action). We do not believe that the questionnaires provided examine such controversial issues. However, if any aspect of this study makes you feel uncomfortable, you may choose not to answer certain questions, or to withdraw from the study at any time.

Benefits of the Study: We anticipate that you will benefit from this study by learning about ways that psychological research can contribute to learning and problem-solving. When the session is over, we will describe the purpose and hypotheses of the study to you in more detail. Please note that some goals of the study will not be revealed to you until the end of your study session. Once we have analyzed the data, you are welcome to ask about the results. However, we cannot guarantee that you will receive any direct benefits from participating in this study.

Confidentiality: Your responses in this research will remain strictly confidential. You will not put your name or student number on the questionnaires. Rather, the questionnaires will be identified by number only. You will be asked to sign only this consent form (if you decide to participate), and it will be filed 
separately from your questionnaires. The data from this study will be held in a locked lab room in Dr. Burke's lab, to which only investigators, supervising faculty, and research assistants will have access. The data will be kept for five years, after which time it will be destroyed.

Incentives to Participate: Although we appreciate the contributions of participants in our research, you will not be paid for your participation in this study. However, you will receive a $1 \%$ course credit toward the 3\% research participation mark in your Introductory Psychology course. If you would prefer to walk through the study (that is, if you would like to observe the research process but not provide any personal data), you will still be given $1 \%$ course credit.

Voluntary Nature of Participation: Participation in this study is voluntary. Your choice of whether or not to participate will not affect your grades or academic status or your future relations with the University. If you decide to participate, you are free to withdraw your consent and to stop your participation at any time without penalty or loss of benefits to which you are allowed. Should you withdraw from the study, you will still be given $1 \%$ course credit (provided that you have not already received the maximum allotted for research participation of $3 \%$ ).

Questions about the Study: If you have any questions about the research now, please ask. If you have questions later about the research, you may contact:

Stephanie Marion, smarion@psych.ryerson.ca, 416-979-5000, ext 2190

Dr. Tara Burke, tburke@psych.ryerson.ca, 416-979-5000, ex. 6519

If you have any questions regarding your rights as a human participant in this study, you may contact the Ryerson University Research Ethics Board for information: Toni Fletcher, toni.fletcher@ryerson.ca (416) 979-5000 ext 7112.

Agreement: Your signature below indicates that you have read the information in this agreement and have had a chance to ask any questions you have about the study. Your signature also indicates that you agree to be in the study and have been told that you can change your mind any time during the study and draw from it.

You have been told that by signing this consent agreement, you are not giving up any of your legal rights.

$\overline{\text { Name of Participant (please print) }}$

Signature of Participant

Date

Signature of Experimenter

Date 


\section{Liking Questionnaire}

Please rate how much you agree or disagree with the following statements about the other participant with whom you will complete a collaborative task:

1. I like the participant that I am assigned to work with

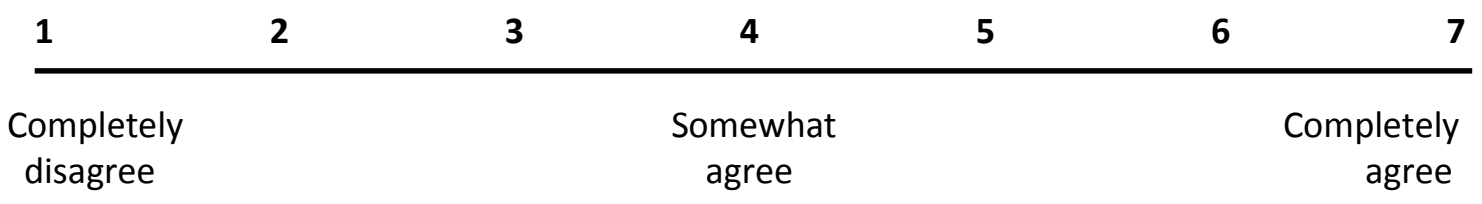

2. I dislike the participant I am assigned to work with

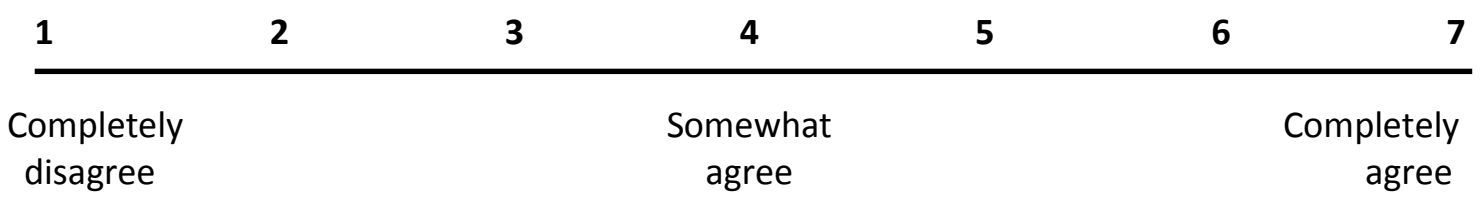

3. I would exchange email addresses with the participant I am assigned to work with

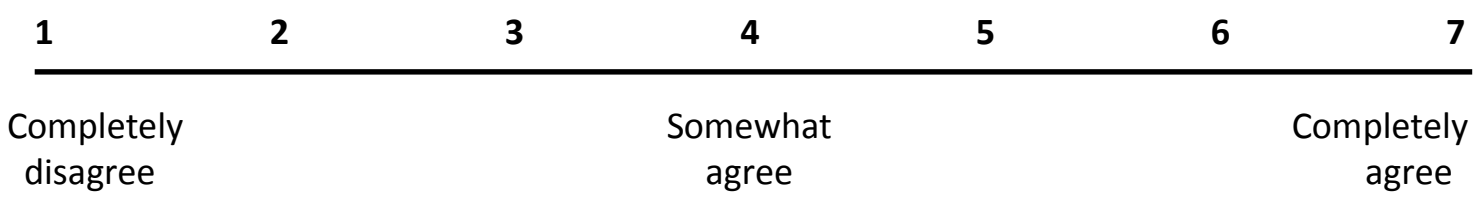

4. I would go to a party with the other participant if he/she invited me

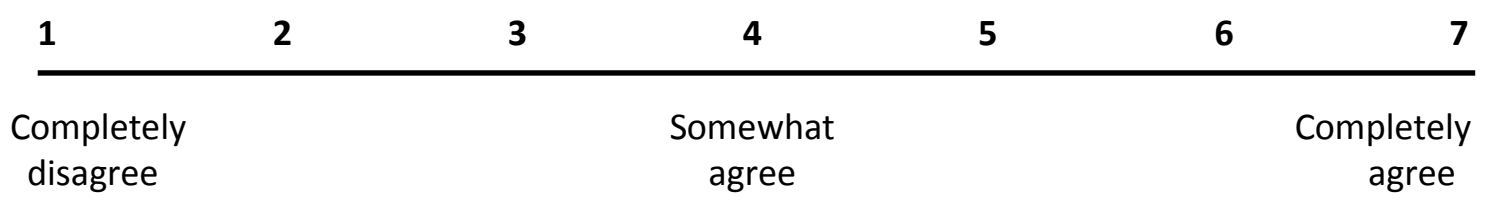

5. I would go sit beside the other participant if I saw him/her in a lecture

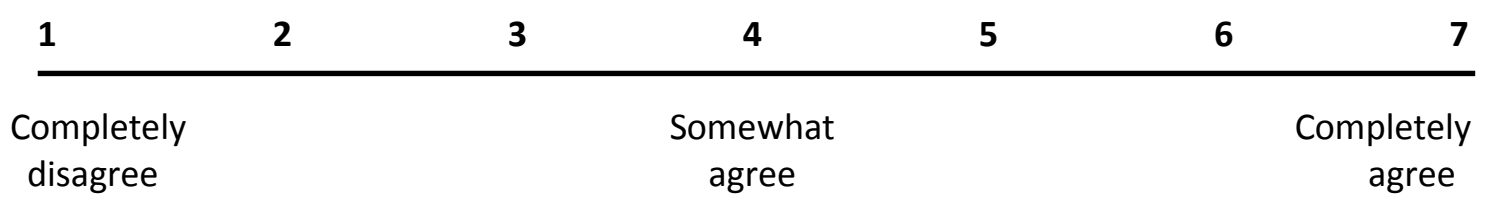


6. I would say hi to the other participant if I crossed them in the hallway

\begin{tabular}{ccccccr}
$\mathbf{1}$ & $\mathbf{2}$ & $\mathbf{3}$ & $\mathbf{4}$ & $\mathbf{5}$ & $\mathbf{6}$ & $\mathbf{7}$ \\
\hline $\begin{array}{c}\text { Completely } \\
\text { disagree }\end{array}$ & & $\begin{array}{c}\text { Somewhat } \\
\text { agree }\end{array}$ & & Completely \\
agree
\end{tabular}

7. I would accept a Facebook invitation from the other participant

\begin{tabular}{lllllrr}
$\mathbf{1}$ & $\mathbf{2}$ & $\mathbf{3}$ & $\mathbf{4}$ & $\mathbf{5}$ & $\mathbf{6}$ & $\mathbf{7}$ \\
\hline $\begin{array}{c}\text { Completely } \\
\text { disagree }\end{array}$ & \multicolumn{3}{c}{$\begin{array}{c}\text { Somewhat } \\
\text { agree }\end{array}$} & & Completely \\
agree
\end{tabular}

8. I believe I will work well with the other participant

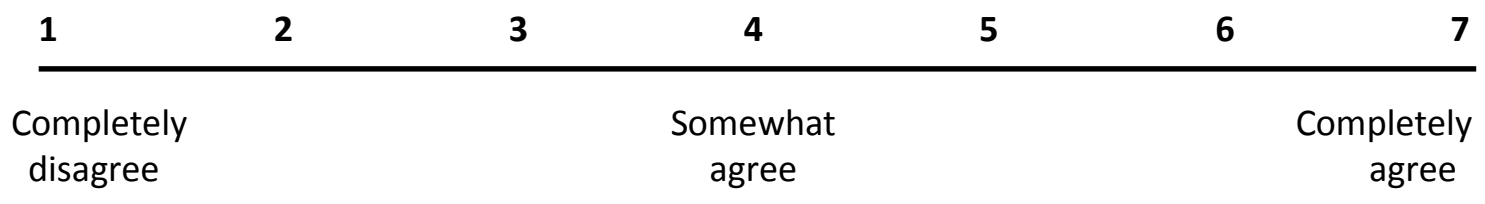

9. I would enjoy time spent with this person

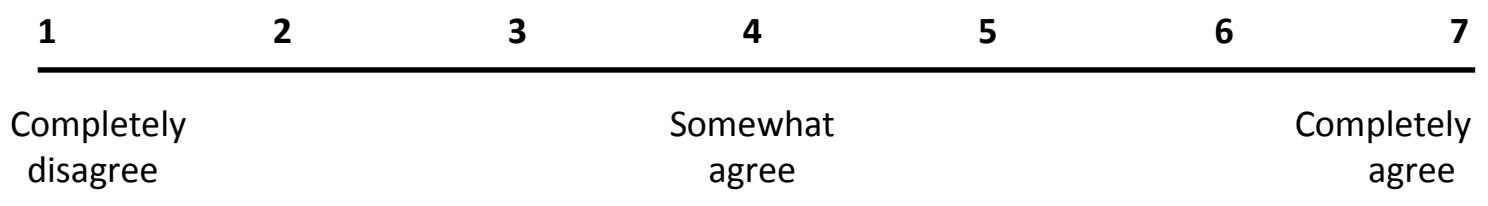

10. The other participant is friendly

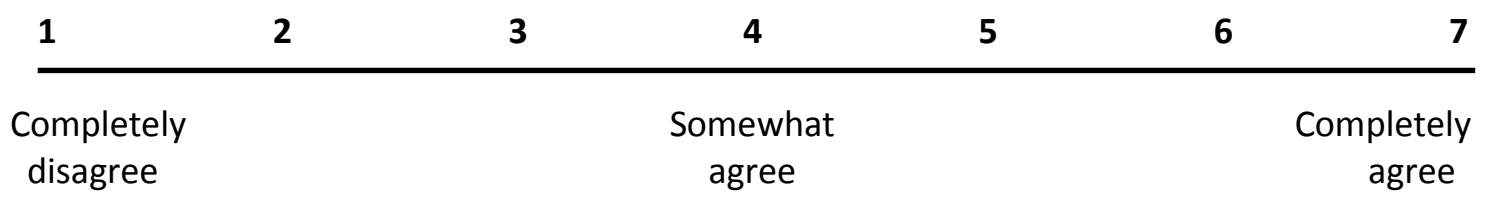




\section{Debriefing Questionnaire}

1. How suspicious were you of the confederate? (i.e., that she stole the money)

$\begin{array}{ccccccccc}1 & 2 & 3 & 4 & 5 & 6 & 7 & 8 & 9 \\ \text { (sure she/he didn't do it) } & & & & & & \begin{array}{l}10 \\ \text { (sure that she/he did it) }\end{array}\end{array}$

2. Did you hear the confederate say "we were in here the whole time"? Yes $\square$

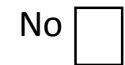

3. Why did you or did you not say anything on the spot when she/he said that?

Momentarily forgot that she/he went to the washroom

Did not want to get involved

Did not want to say anything in front of her/him

Didn't think she/he would have done it

Confed. asked not to say she/he left

Other:

4. Why did you say what you said when I asked you if she/he was here the whole time?

5. Did she/he ask you not to report that she/he had left the room?

6. Why did you support or not support her alibi that she/he was here the whole time?

7. Did you ever think that maybe you should/should not tell $E$ that she/he went to the washroom?

8. Did you ever think that maybe you should/should not tell me that she/he asked you not to tell me she/he left?

9. Thinking back to just after you finished the last group task ("memory task"), how would you rate your liking of the confederate?

$\begin{array}{lllllll}1 & 2 & 3 & 4 & 5 & 6 & 7\end{array}$




\section{Debriefing Form for Study 1}

Thank you for participating in this experiment. As you may recall, you were told that the aim of this study was to examine how similar and dissimilar personality characteristics affect problem-solving skills. However, the actual purpose of this research is to examine the impact of interpersonal relationship on alibi corroboration. An alibi is a type of statement that a person suspected of a crime provides to police to indicate that he or she could not have committed the crime due to being elsewhere from where the crime was committed. Sometimes such statements are corroborated (or confirmed) by another witness. In this study, we want to know whether people who feel closer to one another (such as friends) are more likely to support one another's alibi, even if the alibi provided is false.

Courts seem to think that people lie for one another, and especially those who are close to one another. This assumption has important implications for the believability of a defendant's alibi in court. This study examines the validity of this assumption. To simulate different degrees of relationship, the affiliation between the two participants (an independent variable) was manipulated in this experiment. The other participant in this study was actually a confederate - someone who is aware of the experiment and is helping out the experimenter. While all participants completed the same tasks, some completed the first one alone, and others completed it with the confederate. The participants who did the first task alone were told that their personality was dissimilar to that of the confederate, and those who did the first task in pairs were told that their personality was similar to that of the confederate. This was done in order to create feelings of unfamiliarity in the first case, or feelings of friendship in the second case.

We hypothesized that participants who have been involved in friendship-enhancing activities will be more likely, when questioned alone, to corroborate the confederate's alibi than those who remained strangers. That is, they would be more likely to agree that the confederate remained in the study room during the entire session (the dependent variable).

The purpose of the study is to understand how personal affiliations to others influence the likelihood of supporting a statement that may not be completely true. The results will contribute to our theoretical understanding of alibi corroboration and perception, and will allow us to make recommendations to the police regarding their ability to assess the credibility of alibis. The results also have implications for police investigative processes and for determining factors involved in wrongful convictions.

As I'm sure you noticed there was some deception in this experiment - that is, some aspects of the studies were not exactly as originally described. In particular, there were no other real participants, and the information you received about the purpose of the study was not fully explained to you at the start. The other person involved in this study was a confederate - he or she was a trained lab volunteer. Our main interests were in how you would respond when asked whether the confederate had been in the study room during the entire session. We hope that you understand the reasons why the real purpose of the experiment could not be revealed to you until now. We couldn't have obtained your unbiased reactions to the situations if you had known exactly what we were interested in. As a result, we had to set up somewhat contrived situations.

We would like to remind you that your results are confidential to the experimenters and that all results are published anonymously as a group. However, if you feel uncomfortable about any aspect of the study, you are free to request to have your data withdrawn from the sample. Also, if you know of others who will be participating in this experiment, please refrain from discussing it with them. We do not want our future participants to be aware of the procedures and expected findings. 
We would like to thank you again for your participation in our study. Please retain your consent form for future reference and feel free to contact the student experimenter of Professor Burke if you have any further questions or concerns, or if you would like any information about the results once it is completed:

\begin{tabular}{|c|c|}
\hline $\begin{array}{c}\text { Stephanie Marion, B.Sc. } \\
\text { Graduate Student }\end{array}$ & Tara Burke, Ph.D. \\
Ryerson University & Department of Psychology \\
Ryarion@psych.ryerson.casm & Ryerson University \\
t16-979-5000, ext 2190 & tburke@psych.ryerson.ca \\
\hline
\end{tabular}




\section{APPENDIX B: CODING MANUAL FOR DEBRIEFING QUESTIONS}

"P" = Participant, $\quad$ " $C$ " = Confederate, $\quad$ "E" = Experimenter

\section{1) Responses relating to the alibi questions (Study 1: Qs 4 \& 6; Studies 2 \& 3: Qs 4 \& 5):}

Participant-centered responses:

- "Morality-based response":

When $P$ says that (s)he reported absence to $E$ because it is the 'right thing' to do, because stealing is wrong, because lying is wrong, because (s)he feels empathy for $E$, thought $E$ should know, etc.

- "Avoiding confrontation/involvement":

When $\mathrm{P}$ says that ( $\mathrm{s}$ )he did not report C's absence in order to avoid a conflict or avoid getting involved in the situation, or did report the absence to avoid a confrontation if ( $\mathrm{s}$ )he gets caught in a lie

- "Memory lapse":

When $\mathrm{P}$ claims that (s)he did not report absence because (s)he had forgotten it

- "Caught off guard":

When P says that (s)he did or did not report C's absence because (s)he was caught off guard, was shocked, hadn't had time to process what was going on, etc.

\section{Confederate-centered responses:}

- "Request from confederate":

- "Protection of C or relationship":

- "Lack of trust in C":

- "Trust in C":

\section{Situation-centered responses:}

When $\mathrm{P}$ does not report that $\mathrm{C}$ left because $\mathrm{C}$ had asked him/her not to report his/her absence

When $\mathrm{P}$ says that (s)he did not report absence in order to protect $\mathrm{C}$, to not be a 'rat', to maintain the trust of $\mathrm{C}$, or to protect the relationship in another way

When $P$ does or does not report C's absence because (s)he does not know $C$, does not trust $C$, or is suspicious of $C$

When P says that (s)he did or did not report C's absence because (s)he trusts $C$ and does not believe that $C$ was involved
- "Irrelevance":

- "Compliance":
When P says that (s)he did or did not report that C's absence because (s)he thought it was irrelevant, either not important to report, or not anything wrong and therefore no reason NOT to report it

When $P$ says that (s)he reported absence simply because (s)he was asked directly or specifically 


\section{APPENDIX C}

Participants' explanations for rejection and corroboration of the alibi by relationship and request conditions. Note that many participants provided more than one answer.

\begin{tabular}{|c|c|c|c|c|c|c|c|c|c|c|c|}
\hline \multirow[b]{3}{*}{ Response categories } & \multicolumn{5}{|c|}{ Participants who did not corroborate the alibi $(n=46)$} & \multicolumn{5}{|c|}{ Participants who corroborated the alibi $(n=33)$} & \multirow{3}{*}{ Total } \\
\hline & \multicolumn{2}{|c|}{ Relationship Condition } & \multicolumn{2}{|c|}{ Request Condition } & \multirow[b]{2}{*}{ Total } & \multicolumn{2}{|c|}{ Relationship Condition } & \multicolumn{2}{|c|}{ Request Condition } & \multirow[b]{2}{*}{ Total } & \\
\hline & $\begin{array}{c}\mathrm{FE} \\
(n=25)\end{array}$ & $\begin{array}{c}\text { SM } \\
(n=21)\end{array}$ & $\begin{array}{l}\text { Request } \\
(n=15)\end{array}$ & $\begin{array}{c}\mathrm{NR} \\
(n=31)\end{array}$ & & $\begin{array}{c}\mathrm{FE} \\
(n=14)\end{array}$ & $\begin{array}{c}\text { SM } \\
(n=19)\end{array}$ & $\begin{array}{l}\text { Request } \\
(n=23)\end{array}$ & $\begin{array}{c}\mathrm{NR} \\
(n=10)\end{array}$ & & \\
\hline \multicolumn{12}{|l|}{ Participant-centered } \\
\hline Morality-based response & 22 & 16 & 12 & 26 & 38 & -- & -- & -- & -- & -- & 38 \\
\hline $\begin{array}{l}\text { Avoiding confrontation or } \\
\text { involvement }\end{array}$ & 1 & 1 & 2 & -- & 2 & 5 & 4 & 6 & 3 & 9 & 11 \\
\hline Memory lapse & -- & -- & -- & -- & -- & 2 & 4 & 2 & 4 & 6 & 6 \\
\hline Caught off guard & 1 & -- & -- & 1 & 1 & 2 & 1 & 3 & -- & 3 & 4 \\
\hline \multicolumn{12}{|l|}{ Confederate-centered } \\
\hline Request from confederate & -- & -- & -- & -- & -- & 3 & 6 & 9 & -- & 9 & 9 \\
\hline $\begin{array}{l}\text { Protection of confederate } \\
\text { or relationship }\end{array}$ & -- & -- & -- & -- & -- & 7 & 9 & 13 & 3 & 16 & 16 \\
\hline Lack of trust in confederate & 4 & 8 & 1 & 11 & 12 & -- & -- & -- & -- & -- & 12 \\
\hline Trust in the confederate & 4 & 2 & -- & 6 & 6 & 5 & 6 & 8 & 3 & 11 & 17 \\
\hline \multicolumn{12}{|l|}{ Situation-centered } \\
\hline Compliance & 2 & 4 & 2 & 4 & 6 & -- & -- & -- & -- & -- & 6 \\
\hline Irrelevance/innocuous & 5 & 4 & 1 & 8 & 9 & 4 & 6 & 5 & 5 & 10 & 19 \\
\hline
\end{tabular}


APPENDIX D: MATERIAL FOR STUDY 2

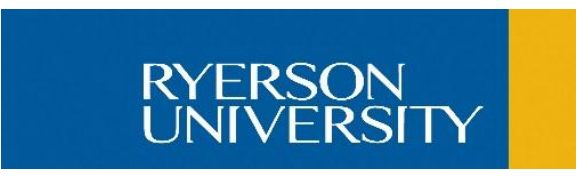

Department of Psychology

\title{
Participants needed for Psychology Research Study:
}

\author{
"Does Personality Affect Group Problem-Solving?" \\ A study looking at the impact of personality and collaboration on \\ problem-solving performance
}

This psychology study examines the impact of personality similarity on individual and collaborative problem-solving. Participants will complete personality questionnaires and sets of problem-solving tasks alone or with another participant.

Eligibility: You must be:

- a Ryerson University student NOT currently enrolled in either PSY102 or PSY202, and:

- NOT majoring in psychology.

Location: Ryerson Psychology Research and Training Centre, 105 Bond Street (SBB building), $2^{\text {nd }}$ floor

Duration: 1 hour: Study sessions are held Mon-Fri between the hours of 10am and $6 \mathrm{pm}$.

Compensation: You will be given $\$ 10$ as a token of our appreciation for your participation

\section{If interested, please contact Stephanie: smarion@psych.ryerson.ca OR 416-979-5000, ext. 2190}


SONA advertisement for study 2

Study Name: A Study on the Social Factors that Impact Problem-Solving Ability - FEMALES ONLY/MALES ONLY

\section{Abstract:}

This 60-min social/cognitive study examines the impact of personality similarity on individual and group problem-solving. Participants will complete personality questionnaires and sets of problem-solving tasks alone or with another participant.

\section{Description:}

Participants will be students at Ryerson University who are currently enrolled in an introductory psychology course (PSY102 or PSY202). We are hoping to recruit 100 participants for this study. After signing up for this study, please arrive at 105 Bond Street at your scheduled time, and wait for the experimenter in the $2^{\mathrm{ND}}$ FLOOR LOBBY (the seating area located by the stairs and elevator). The session will be conducted with two participants at a time. Participants will complete a set of cognitive tasks (e.g., anagrams, pattern recognition, logical reasoning, etc), and will answer some personality questionnaires and some questions about your problemsolving strategies. Some of the tasks will be done individually and some you will be doing collaboratively with another participant. Part of the study session will be audio-recorded, and you may be asked to provide a physiological measure (pulse) while completing some of the tasks. The results of this study will help us better understand human behaviour and collaboration. Minimal anticipated risks are involved. It is possible that you may feel slight discomfort in answering some questions relating to your beliefs or personal opinions. Participating in a study may be a new experience for you, and you can stop your participation either temporarily or permanently at any point without penalty. You are also free not to answer any and all questions you feel uncomfortable answering without penalty.

If you are participating on weekdays after 6PM, or on weekends, the main building doors may be locked. If that is the case, please call 416-979-5000 ext. 2190 and the researcher will let you in. Please note that if you arrive more than 5 minutes late to the study session, you will not be able to participate. 
Consent Form for Study 2

\section{Does Personality Affect Group Problem-Solving?}

You are being asked to participate in a research study. Before signing this consent form, it is important that you read the following information. You may ask as many questions as necessary to be sure that you understand what the study entails.

Investigators:

\begin{tabular}{|c|c|}
\hline Stephanie Marion, B.Sc., M.A. & Tara Burke, Ph.D. \\
Graduate Student & Department of Psychology \\
Ryerson University & Ryerson University \\
\hline
\end{tabular}

Purpose of the Study: The primary purpose of this study is to examine how group performance on certain cognitive tasks is impacted by the similarity (or dissimilarity) between group members' personalities. We are hoping to recruit approximately 100 students to participate in this research.

Description of the Study: If you decide to participate in the research, you will be asked to complete some mood and personality questionnaires and to complete a series of anagram, math and probability, pattern recognition, and logical reasoning problems. Some of these tasks you will do individually and some you will be doing with another participant. Your responses to part of this study will require audio-recording. You may also be asked to provide a physiological measure during part of this study. This would entail wearing a pulse monitor on your index finger to provide a physiological measure in response to images. This study will take place in a psychology lab at 105 Bond St and will take approximately 60 minutes to complete.

What is Experimental in this Study: None of the procedures or questionnaires used in this study are experimental in nature, in the sense that they have all been used in previous cognitive and personality research. From a technical or procedural point of view, part of this study is considered "experimental", because by following the procedure described above, the study examines the impact of one variable (called the "independent variable") on another variables (called the "dependent variable"). More information about the independent and dependent variable will be provided at the end of the session.

Risks or Discomforts: Occasionally people feel uncomfortable when answering questionnaires that ask about attitudes and personal opinions toward controversial social issues (e.g., affirmative action). We do not believe that the questionnaires provided examine such controversial issues. However, if any aspect of this study makes you feel uncomfortable, you may choose not to answer certain questions, or to withdraw from the study at any time.

Benefits of the Study: We anticipate that you will benefit from this study by learning about ways that psychological research can contribute to learning and problem-solving. When the session is over, we will describe the purpose and hypotheses of the study to you in more detail. Please note that some goals of the study will not be revealed to you until the end of your study session. Once we have analyzed the data, you are welcome to ask about the results. However, we cannot guarantee that you will receive any direct benefits from participating in this study. 
Confidentiality: Your responses in this research will remain strictly confidential. You will not put your name or student number on the questionnaires. Rather, the questionnaires will be identified by number only. You will be asked to sign only this consent form (if you decide to participate), and it will be filed separately from your questionnaires. The data from this study will be held in a locked lab room in Dr. Burke's lab, to which only investigators, supervising faculty, and research assistants will have access. The data will be kept for five years, after which time it will be destroyed.

Incentives to Participate: Although we appreciate the contributions of participants in our research, you will not be paid for your participation in this study. However, you will receive a 1\% course credit toward the 3\% research participation mark in your Introductory Psychology course, or \$10 if you are not an introductory psychology student. If you would prefer to walk through the study (that is, if you would like to observe the research process but not provide any personal data), or if you wish to withdraw form the study before its completion, for any reason, you will still be given $1 \%$ course credit $/ \$ 10$.

Voluntary Nature of Participation: Participation in this study is voluntary. Your choice of whether or not to participate will not affect your grades or academic status or your future relations with the University. If you decide to participate, you are free to withdraw your consent and to stop your participation at any time without penalty or loss of benefits to which you are allowed. Should you withdraw from the study, you will still be given 1\% course credit (provided that you have not already received the maximum allotted for research participation of $3 \%$ ).

Questions about the Study: If you have any questions about the research now, please ask. If you have questions later about the research, you may contact:

Stephanie Marion, smarion@psych.ryerson.ca, 416-979-5000, ext 2190

Dr. Tara Burke, tburke@psych.ryerson.ca, 416-979-5000, ex. 6519

If you have any questions regarding your rights as a human participant in this study, you may contact the Ryerson University Research Ethics Board for information: Toni Fletcher, toni.fletcher@ryerson.ca (416) 979-5000 ext 7112.

Agreement: Your signature below indicates that you have read the information in this agreement and have had a chance to ask any questions you have about the study. Your signature also indicates that you agree to be in the study and have been told that you can change your mind any time during the study and draw from it.

You have been told that by signing this consent agreement, you are not giving up any of your legal rights.

Signature of Participant

Signature of Experimenter

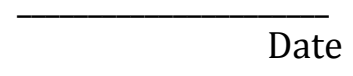

Date 


\section{Individual Problem-Solving Activities}

\# 1.

Gerald who is twelve years old is three times as old as his sister Jane.

How old will Gerald be when he is twice as old as Jane?
a) 13 years
b) 16 years
c) 17 years
d) 18 years
e) 22 years

ANSWER:

\# 2.

If it takes 5 bakers, 250 minutes to make 500 cookies. How many minutes would it take for 2 bakers to make the same amount of cookies?

ANSWER:

\section{\# 3.}

Which number does not belong in the table below?

\begin{tabular}{|c|c|c|}
\hline 17 & 9 & 11 \\
\hline 5 & 87 & 12 \\
\hline 93 & 3 & 15 \\
\hline 77 & 21 & 1 \\
\hline
\end{tabular}

ANSWER:

\#4.

Find four words, horizontally, vertically or diagonally.

$\begin{array}{cccc}\mathbf{F} & \mathbf{M} & \mathbf{Q} & \mathbf{M} \\ \mathbf{T} & \mathbf{O} & \mathbf{U} & \mathbf{R} \\ \mathbf{F} & \mathbf{R} & \mathbf{U} & \mathbf{R} \\ \mathbf{X} & \mathrm{E} & \mathbf{U} & \mathbf{R}\end{array}$

ANSWER: 
\#5.

Rearrange the following letters to make a word and choose the category in which it fits.

\section{"AHHIGNAS"}
a) City
b) Ocean
c) Vegetable
d) Flower

ANSWER

\#6.

Which number should replace $X$ ?

\begin{tabular}{|c|c|c|c|}
\hline 7 & 9 & 4 & 4 \\
\hline 16 & 8 & 3 & 8 \\
\hline 10 & 2 & 2 & 6 \\
\hline 19 & 16 & 7 & $X$ \\
\hline
\end{tabular}

ANSWER:

\#7.

If the words in the following questions were arranged in alphabetical order, then which word would come third?

$$
\text { Jargon - Jaguar - Jacuzzi - Jail - Jazz }
$$

ANSWER: 
\#8.

518119518 is to Eraser as 615182021145 is to...
a) Interest
b) Fortune
c) Money
d) Abandon

ANSWER:

\#9.

How many four sided figures are in this diagram?

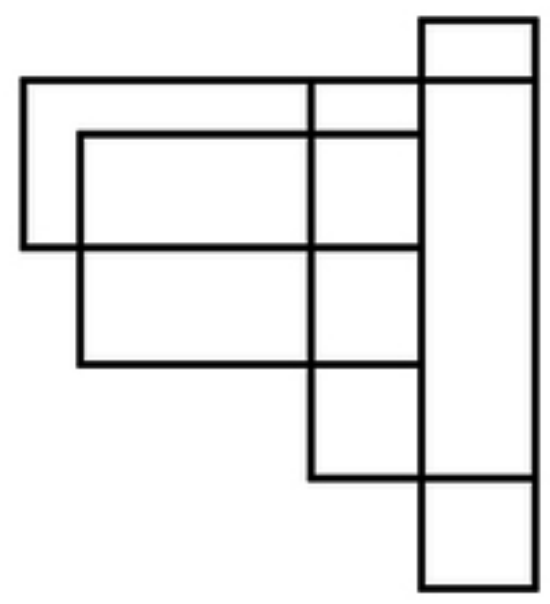
a) 18
b) 20
c) 25
d) 28
e) 32

\section{\#10.}

Put a mathematical symbol between $6 \& 9$ to get a number which is greater than 6 but lesser than 9.

ANSWER: 69 


\section{Individual Problem-Solving Post Questionnaire}

Age:

Gender:

Please rate how much you agree or disagree with each of the following statements, using the scale below:

$1=$ Completely disagree

$2=$ Somewhat disagree

$3=$ Neither agree nor disagree

$4=$ Somewhat agree

5 = Completely agree

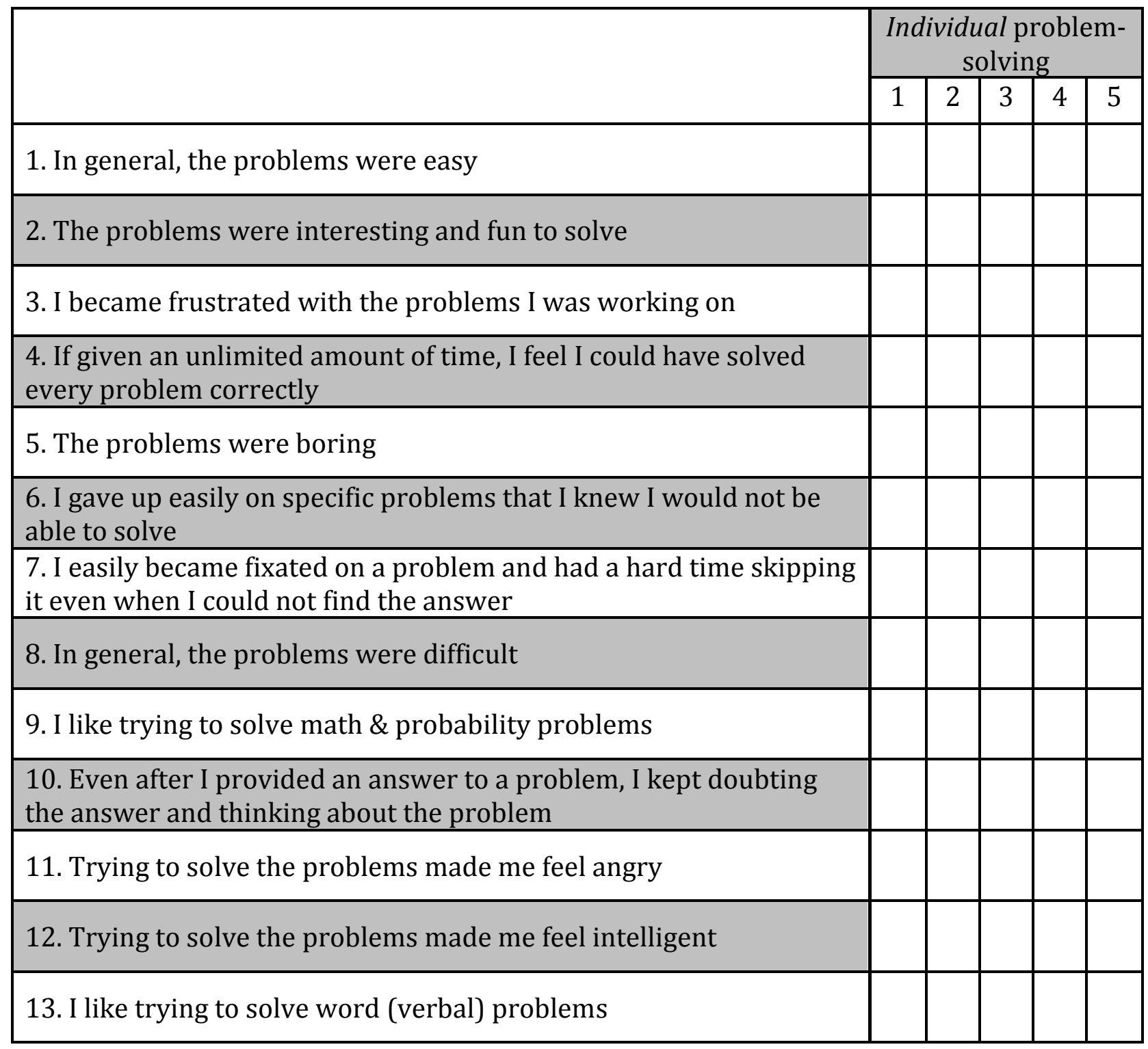


Please rate how much you agree or disagree with the following statements about the other participant with whom you will complete a collaborative task:

1. I think I will like the participant that I am assigned to work with

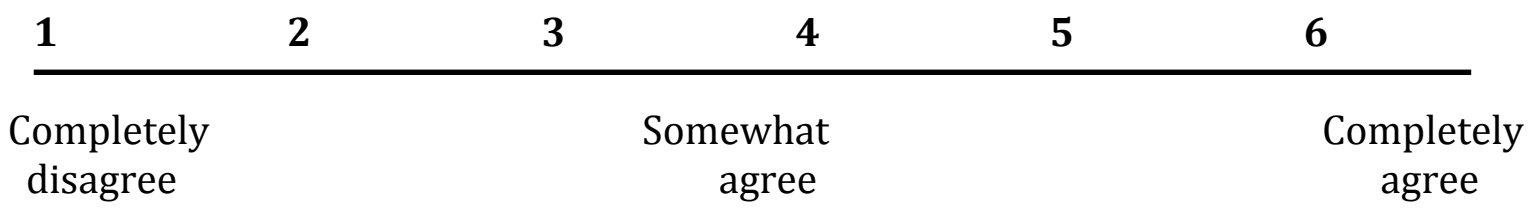

2. I would go sit beside the other participant if I saw him/her in a lecture

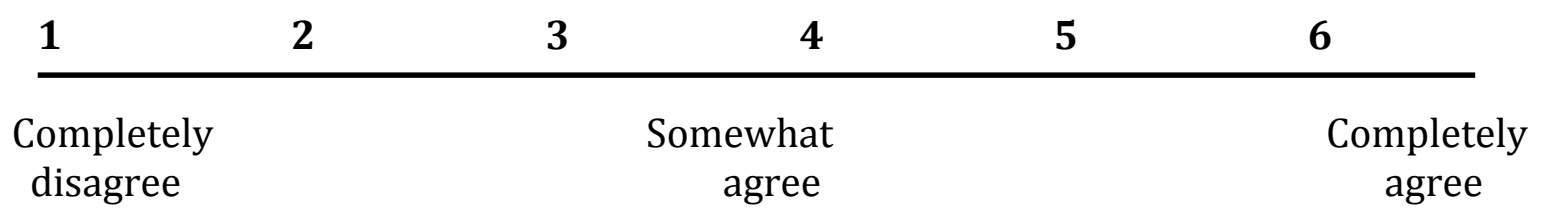

3. I would say hi to the other participant if I crossed them in the hallway

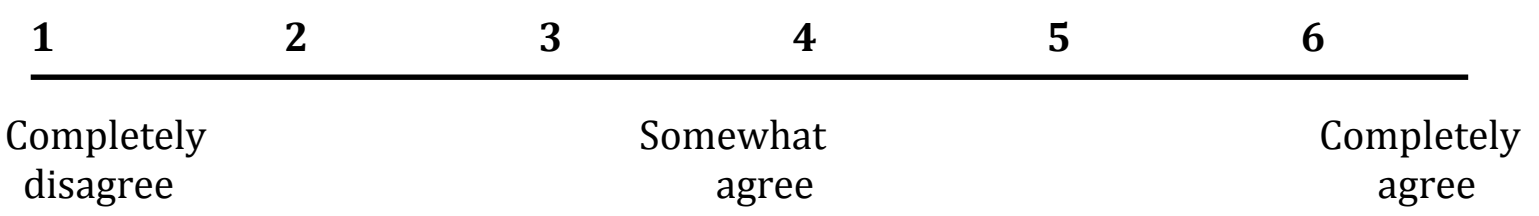

4. I believe I will work well with the other participant

$\begin{array}{cccccc}\mathbf{1} & \mathbf{2} & \mathbf{3} & \mathbf{4} & \mathbf{5} & \mathbf{6} \\ \begin{array}{c}\text { Completely } \\ \text { disagree }\end{array} & & \begin{array}{c}\text { Somewhat } \\ \text { agree }\end{array} & \mathbf{7}\end{array}$

5. The other participant seems friendly

\begin{tabular}{lrrrrr}
$\mathbf{1}$ & $\mathbf{2}$ & $\mathbf{3}$ & $\mathbf{4}$ & $\mathbf{5}$ & $\mathbf{6}$ \\
\hline $\begin{array}{c}\text { Completely } \\
\text { disagree }\end{array}$ & & $\begin{array}{c}\text { Somewhat } \\
\text { agree }\end{array}$ & $\mathbf{7}$
\end{tabular}


Here are a number of personality traits that may or may not apply to you. Please write a number next to each statement to indicate the extent to which you agree or disagree with that statement. You should rate the extent to which the pair of traits applies to you, even if one characteristic applies more strongly than the other.

1 - Disagree strongly

2 - Disagree moderately

3 - Disagree a little

4 - Neither agree nor disagree

5 - Agree a little

6 - Agree moderately

7 - Agree strongly

I see myself as:

1. Extraverted, enthusiastic

2. Critical, quarrelsome.

3. Dependable, self-disciplined.

4. Anxious, easily upset.

5. Open to new experiences, complex.

6. Reserved, quiet.

7. Sympathetic, warm.

8. Disorganized, careless.

9. Calm, emotionally stable.

10. Conventional, uncreative. 


\section{Revised Social Responsibility Scale (Berkowitz \& Daniels, 1964)}

Please rate how much you agree or disagree with each of the following statements:

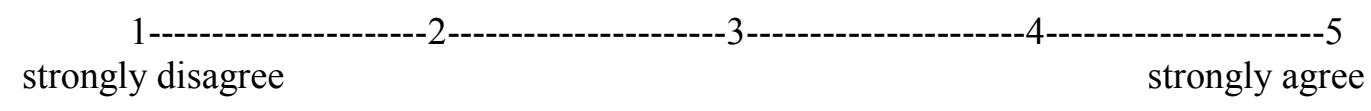

1. It is always important to finish anything that you have started.

2. It is no use worrying about current events or public affairs; I can't do anything about them anyway.

3. In school/work my behavior has gotten me into trouble.

4. I have been in trouble with the law or police.

5. When a person does not tell all his income in order to get out of paying some of his taxes it is just as bad as stealing money from the government.

6. When I work on a committee, I usually let other people do most of the planning.

7. I am often late for school/work.

8. If it is worth starting, it is worth finishing.

9. I am the kind of person that people can count on.

10. I do my chores the very best I know how.

11. When you can't do a job, it is no use to try to find someone else to do it.

12. Why bother to vote when you can do so little with just your one vote.

13. Letting your friends down is not so bad because you can't do good all the time for everybody.

14. Our country would be a lot better off if we didn't have elections and people didn't have to vote.

15. It is more important to work for the good of the team than to work for your own good.

16. I would never let a friend down when he expects something of me.

17. People would be a lot better off if they could live far away from other people and never have anything to do for them.

18. Every person should give some of his time for the good of his town or city.

19. Doing things which are important should come before things you enjoy doing.

20. Cheating on examinations is not so bad as long as nobody ever knows.

21. I usually volunteer for special projects at school/work.

22. When given a task I stick to it even if things I like to do better come along. 


\section{Right-Wing Authoritarianism Scale (Altemeyer, 1981)}

\section{Personality Questionnaire - Match check}

Please complete the following brief personality/opinions questionnaire. Indicate the degree to which you agree or disagree with each statement by circling the appropriate number:

\section{$\begin{array}{lllllllll}-4 & -3 & -2 & -1 & 0 & +1 & +2 & +3 & +4\end{array}$ Very strongly disagree Neutral Very strongly agree}

1. Our country desperately needs a mighty leader who will do what has to be done to destroy the radical new ways and sinfulness that are ruining us.

$\begin{array}{lllllllll}-4 & -3 & -2 & -1 & 0 & +1 & +2 & +3 & +4\end{array}$

2. Gays and lesbians are just as healthy and moral as anybody else.
$-4$
$-3$
$-2-1$
$0+1$
$+2+3+4$

3. It is always better to trust the judgment of the proper authorities in government and religion, than to listen to the noisy rabble-rousers in our society who are trying to create doubt in people's minds.

$\begin{array}{lllllllll}-4 & -3 & -2 & -1 & 0 & +1 & +2 & +3 & +4\end{array}$

4. Atheists and others who have rebelled against the established religions are no doubt every bit as good and virtuous as those who attend church regularly.

$\begin{array}{lllllllll}-4 & -3 & -2 & -1 & 0 & +1 & +2 & +3 & +4\end{array}$

5. The only way our country can get through the crisis ahead is to get back to our traditional values, put some tough leaders in power, and silence the troublemakers spreading bad ideas.

$\begin{array}{llllllll}-4 & -3 & -2 & -1 & 0 & +1 & +2 & +3\end{array}$

6. There is absolutely nothing wrong with nudist camps.

$\begin{array}{lllllllll}-4 & -3 & -2 & -1 & 0 & +1 & +2 & +3 & +4\end{array}$

7. Our country needs free thinkers who will have the courage to defy traditional ways, even if this upsets many people.

$\begin{array}{lllllllll}-4 & -3 & -2 & -1 & 0 & +1 & +2 & +3 & +4\end{array}$

8. Our country will be destroyed someday if we do not smash the perversions eating away at our moral fibre and traditional beliefs.

$\begin{array}{lllllllll}-4 & -3 & -2 & -1 & 0 & +1 & +2 & +3 & +4\end{array}$

9. Everyone should have their own lifestyle, religious beliefs, and sexual preferences, even if it makes them different from everyone else.

$\begin{array}{lllllllll}-4 & -3 & -2 & -1 & 0 & +1 & +2 & +3 & +4\end{array}$ 
10. The "old-fashioned ways" and "old-fashioned values" still show the best way to live.

$\begin{array}{lllllllll}-4 & -3 & -2 & -1 & 0 & +1 & +2 & +3 & +4\end{array}$

11. You have to admire those who challenged the law and the majority's view by protesting for abortion rights, for animal rights, or to abolish school prayer.

$\begin{array}{lllllllll}-4 & -3 & -2 & -1 & 0 & +1 & +2 & +3 & +4\end{array}$

12. What our country really needs is a strong, determined leader who will crush evil, and take us back to our true path.

$\begin{array}{lllllllll}-4 & -3 & -2 & -1 & 0 & +1 & +2 & +3 & +4\end{array}$

13. Some of the best people in our country are those who are challenging our government, criticizing religion, and ignoring the "normal way things are supposed to be done."

$\begin{array}{lllllllll}-4 & -3 & -2 & -1 & 0 & +1 & +2 & +3 & +4\end{array}$

14. God's laws about abortion, pornography, and marriage must be strictly followed before it is too late, and those who break them must be strongly punished.

$\begin{array}{llllllll}-4 & -3 & -2 & -1 & 0 & +1 & +2 & +3\end{array}$

15. There are many radical, immoral people in our country today, who are trying to ruin it for their godless purposes, who the authorities should put out of action.

$\begin{array}{lllllllll}-4 & -3 & -2 & -1 & 0 & +1 & +2 & +3 & +4\end{array}$

16. A "woman's place" should be wherever she wants to be. The days when women are submissive to their husbands and social conventions belong strictly in the past.

$\begin{array}{lllllllll}-4 & -3 & -2 & -1 & 0 & +1 & +2 & +3 & +4\end{array}$

17. Our country will be great if we honour the ways of our forefathers, do what the authorities tell us to do, and get rid of the "rotten apples" who are ruining everything.

$\begin{array}{llllllll}-4 & -3 & -2 & -1 & 0 & +1 & +2 & +3\end{array}+4$

18. There is no "ONE right way" to live life; everybody has to create their own way.

$\begin{array}{llllllll}-4 & -3 & -2 & -1 & 0 & +1 & +2 & +3\end{array}$

19. Homosexuals and feminists should be praised for being brave enough to defy "traditional family values."

$\begin{array}{lllllllll}-4 & -3 & -2 & -1 & 0 & +1 & +2 & +3 & +4\end{array}$

20. This country would work a lot better if certain groups of troublemakers would just shut up and accept their group's traditional place in society.

$\begin{array}{lllllllll}-4 & -3 & -2 & -1 & 0 & +1 & +2 & +3 & +4\end{array}$

21. It would be best for everyone if the proper authorities censored magazines so that people could not get their hands on trashy and disgusting material.

$\begin{array}{llllllll}-4 & -3 & -2 & -1 & 0 & +1 & +2 & +3\end{array}+4$ 
22. There is nothing wrong with premarital sexual intercourse.
$-4$
$-1$
$0+1+2+3+4$

23. People should pay less attention to the Bible and the other forms of religious guidance, and instead develop their own personal standards of what is moral and immoral.
$-4$
$-3$
$-2$
$0+1$
$+2+3+4$

24. What our country needs most is discipline, with everyone following our leaders in unity.
$-$
$-1$
$0+1$
$+2$
$+3+4$

25. A lot of our rules regarding modesty and sexual behaviour are just customs that are not necessarily any better or holier than those which other people follow.
$-4 \quad-3$
$-2 \quad-1$
$0+1$
$+2+3$
$+4$

26. The facts on crime, sexual immorality, and the recent public disorders all show we have to crack down harder on deviant groups and troublemakers if we are going to save our moral standards and preserve law and order.
$-4$
$-3$
$-2 \quad-1$
$0+1+2$
$+3+4$

27. It's better to have trashy magazines and radical pamphlets in our communities than to let the government have the power to censor them.

$\begin{array}{lllllllll}-4 & -3 & -2 & -1 & 0 & +1 & +2 & +3 & +4\end{array}$

28. The situation in our country is getting so serious, the strongest methods would be justified if they eliminated the troublemakers and got us back on our true path.

$\begin{array}{lllllllll}-4 & -3 & -2 & -1 & 0 & +1 & +2 & +3 & +4\end{array}$

29. It is wonderful that young people today have greater freedom to protest against things they don't like, and to make their own "rules" to govern their behaviour.

$\begin{array}{llllllll}-4 & -3 & -2 & -1 & 0 & +1 & +2 & +3\end{array}$

30. Once the government leaders give us the "go-ahead," it will be the duty of every patriotic citizen to help stomp out the rot that is poisoning our country from within.
$-2$
$-1$
$0+1$
$+2+3+4$ 


\section{Debriefing questionnaire}

1. How suspicious were you of the confederate before I asked you if he/she was here the whole time? (i.e., that she stole the money)

$\begin{array}{llllllllll}1 & 2 & 3 & 4 & 5 & 6 & 7 & 8 & 9 & 10\end{array}$

(sure she/he didn't do it)

(sure that she/he did it)

2. Did you hear the confederate say "we were in here the whole time"? $\square$ Yes $\square$ No

Comments:

3. Why did you or did you not say anything on the spot when she said that?

Momentarily forgot that she/he went to the washroom

Did not want to get involved

Did not want to say anything in front of her/him

Didn't think she would have done it

Confed. asked not to say he/she left

Other:

4. People in this study respond in various ways and for various reasons. Why did you respond the way you did when I asked you if he/she was here the whole time?

5. Why did you support/not support his/her alibi that he/she was here the whole time?

6. Did you ever think that maybe you should/should not have told me that she/he went to the washroom? 
Thank you for participating in this experiment. As you may recall, you were told that the aim of this study was to examine how the similarity of personality characteristics can affect problem-solving skills. However, the actual purpose of this research is to examine the social reciprocity norm in an alibi corroboration context. The reciprocity norm states that individuals are motivated to help those who have helped them in the past (the "tit for tat" norm). An alibi is a type of statement that a person suspected of a crime provides to police to indicate that he or she could not have committed the crime due to being elsewhere from where the crime was committed. Sometimes such statements are corroborated (or confirmed) by another witness. In this study, we want to know whether people who previously received helped from another person are later more likely to support that person's alibi, even if the alibi provided is false.

Courts seem to think that people lie for one another, and especially those who are close to one another or those who belong to the same social groups. One reason for this assumption may be that those who are part of the same groups may have had interactions in the past (e.g., previous situation where one person helped another) that would motivate these individuals to help each other at a later time. This assumption has important implications for the believability of a defendant's alibi in court. This study examines the validity of this assumption. To simulate different degrees of the salience of the reciprocity norm, helping behaviour (the independent variable) was manipulated in this experiment. The other participant in this study was actually a confederate - someone who is aware of the experiment and is helping out the experimenter. While all participants completed the same tasks, sometimes the confederate helped the participant complete the difficult cognitive tasks (in the 'helping condition'), and sometimes the confederate did not help the participant (in the 'no-help condition').

The 'alibi' in this study consisted of the confederate's claim that he or she was in the testing room the entire time, and therefore could not have been involved in the supposed money theft (however no such theft actually occurred). We hypothesized that participants who are helped complete their task (and who are therefore more likely to win a prize) will be more likely, when questioned alone, to corroborate the confederate's alibi than those who are not helped by the confederate. That is, they would be more likely to agree that the confederate remained in the study room during the entire session (the dependent variable).

The purpose of the study is to understand how personal interactions influence the likelihood of supporting a statement that may not be completely true. The results will contribute to our theoretical understanding of alibi corroboration and perception, and may allow us to make recommendations to the police regarding the extent to which an alibi corroboration should or should not be believed. The results also have implications for police investigative processes and for determining factors involved in wrongful convictions.

As I'm sure you noticed there was some deception in this experiment - that is, some aspects of the studies were not exactly as originally described. In particular, there were no other real 
participants, and the information you received about the purpose of the study was not fully explained to you at the start. The other person involved in this study was a confederate - he or she was a trained lab volunteer. Our main interests were in how you would respond when asked whether the confederate had been in the study room during the entire session. We hope that you understand the reasons why the real purpose of the experiment could not be revealed to you until now. We couldn't have obtained your unbiased reactions to the situations if you had known exactly what we were interested in. As a result, we had to set up somewhat contrived situations.

We would like to remind you that your results are confidential to the experimenters and that all results are published anonymously as a group. However, if you feel uncomfortable about any aspect of the study, you are free to request to have your data withdrawn from the sample. Also, if you know of others who will be participating in this experiment, please refrain from discussing it with them. We do not want our future participants to be aware of the procedures and expected findings.

We would like to thank you again for your participation in our study. Please retain your consent form for future reference and feel free to contact the student experimenter of Professor Burke if you have any further questions or concerns, or if you would like any information about the results once it is completed:

\begin{tabular}{|c|c|}
\hline Stephanie Marion, B.Sc., M.A. & Tara Burke, Ph.D. \\
Graduate Student & Department of Psychology \\
Ryerson University & Ryerson University \\
smarion@psych.ryerson.ca & tburke@psych.ryerson.ca \\
\hline $16-979-5000$, ext 2190 & 416-979-5000, ex. 6519 \\
\hline
\end{tabular}




\section{APPENDIX E}

Participants' explanations for rejection and corroboration of the alibi, by help condition and by gender. Note that many participants provided more than one answer.

\begin{tabular}{|c|c|c|c|c|c|c|c|c|c|c|c|}
\hline \multirow[b]{3}{*}{ Response categories } & \multicolumn{5}{|c|}{ Participants who did not corroborate the alibi $(n=56)$} & \multicolumn{5}{|c|}{ Participants who corroborated the alibi $(n=25)$} & \multirow{3}{*}{ Total } \\
\hline & \multicolumn{2}{|c|}{ Help Condition } & \multicolumn{2}{|c|}{ Gender } & \multirow[b]{2}{*}{ Total } & \multicolumn{2}{|c|}{ Help Condition } & \multicolumn{2}{|c|}{ Gender } & \multirow[b]{2}{*}{ Total } & \\
\hline & $\begin{array}{c}\mathrm{PH} \\
(n=28)\end{array}$ & $\begin{array}{c}\mathrm{NH} \\
(n=28)\end{array}$ & $\begin{array}{l}\text { Males } \\
(n=22)\end{array}$ & $\begin{array}{l}\text { Females } \\
(n=34)\end{array}$ & & $\underset{(n=13)}{\mathrm{PH}}$ & $\begin{array}{c}\mathrm{NH} \\
(n=12)\end{array}$ & $\begin{array}{l}\text { Males } \\
(n=15)\end{array}$ & $\begin{array}{l}\text { Females } \\
(n=10)\end{array}$ & & \\
\hline \multicolumn{12}{|l|}{ Participant-centered } \\
\hline Morality-based response & 23 & 21 & 17 & 27 & 44 & -- & -- & -- & -- & -- & 44 \\
\hline $\begin{array}{l}\text { Avoiding confrontation or } \\
\text { involvement }\end{array}$ & 3 & 5 & 3 & 5 & 8 & -- & 1 & 1 & -- & 1 & 9 \\
\hline Memory lapse & -- & -- & -- & -- & -- & 6 & 9 & 7 & 8 & 15 & 15 \\
\hline Caught off guard & 1 & 1 & -- & 2 & 2 & 3 & 3 & 5 & 1 & 6 & 8 \\
\hline \multicolumn{12}{|l|}{ Confederate-centered } \\
\hline $\begin{array}{l}\text { Protection of confederate } \\
\text { or relationship }\end{array}$ & -- & -- & -- & -- & -- & 2 & 2 & 2 & 2 & 4 & 4 \\
\hline Lack of trust in confederate & 11 & 4 & 8 & 7 & 15 & -- & -- & -- & -- & -- & 15 \\
\hline Trust in the confederate & 4 & 3 & 2 & 5 & 7 & 7 & 2 & 6 & 3 & 9 & 16 \\
\hline \multicolumn{12}{|l|}{ Situation-centered } \\
\hline Compliance & 6 & 6 & 3 & 9 & 12 & -- & -- & -- & -- & -- & 12 \\
\hline Irrelevance/innocuous & -- & 1 & -- & 1 & 1 & 4 & 1 & 4 & 1 & 5 & 6 \\
\hline
\end{tabular}




\section{APPENDIX F: MATERIALS FOR STUDY 3}

Recruitment Advertisement for Study 3 (Friends Condition)

Study Name: A Study on Friends' Performance on Problem-Solving *MUST BRING
FRIEND*

Abstract:

This 60min social/cognitive study looks at how personality and interpersonal closeness affects problem-solving skills. *PARTICIPANTS MUST COME TO THIS STUDY WITH A FRIEND* You will complete personality measures and tasks alone or with a friend.

\section{Description:}

Participants will be students at Ryerson University who are currently enrolled in an introductory psychology course, and friends of such students. 68 pairs of friends will be tested. The session will be conducted with two participants at a time (one introductory psychology student and a friend who is not currently taking an introductory psychology course). **INTRODUCTORY PSYCHOLOGY STUDENTS WHO SIGN UP FOR THIS STUDY MUST BRING A FRIEND OF THE SAME GENDER TO THE SESSION.** After signing up for this study, please arrive, with your friend, at 105 Bond Street, and wait for the experimenter in the 2ND FLOOR LOBBY (the seating area located by the stairs and elevator). Only the person who signs up for this study on SONA (i.e., the PSY102 or PSY202 student) will receive a course credit for participating. Your friend will receive $\$ 10$ as compensation for their participation. All participants will complete a set of cognitive tasks (e.g., word search, world-scramble, and memory tasks), and will answer questions about their friendship, their personality, and their problem-solving strategies. Some of the tasks will be done individually and some you will be doing collaboratively with your friend. Part of the study session will be audio-recorded, and you may be asked to provide a physiological measure. The results of this study will help us better understand human behaviour and collaboration. Minimal anticipated risks are involved. It is possible that you may feel slight discomfort in answering some questions relating to your beliefs, personal opinions, or friendships. Participating in a study may be a new experience for you, and you can stop your participation either temporarily or permanently at any point without penalty. You are also free not to answer any and all questions you feel uncomfortable answering without penalty. If you are participating on weekdays after 6PM, or on weekends, the main building doors may be locked. If that is the case, please call 416-9795000 ext. 2190 and the researcher will let you in. Please note that participants who arrive late will not be able to complete the study. 
Consent Forms (Friends condition)

\section{A Study on the Social Factors that Impact Friends' Performance on Problem-Solving Tasks}

You are being asked to participate in a research study. Before signing this consent form, it is important that you read the following information, and ask any questions that you may have.

Investigators:

\begin{tabular}{|c|c|}
\hline Stephanie Marion, B.Sc., M.A. & Tara Burke, Ph.D. \\
Graduate Student & Department of Psychology \\
Ryerson University & Ryerson University \\
\hline
\end{tabular}

Purpose of the Study: The primary purpose of this study is to examine how interpersonal closeness and personality characteristics can impact how individuals work together toward a common goal. We are hoping to recruit 132 participants for this study.

Description of the Study: If you decide to participate in the research, you will be asked to do the following: you will be asked to complete a personality questionnaire, a short demographics questionnaire, a friendship questionnaire, a series of cognitive problem-solving tasks (e.g., a wordgeneration task, a word scramble task and a memory task), and a problem-solving strategies questionnaire. Some of these tasks you will do individually and some you will be doing with your friend. Some of your responses to part of this study may require audio-recording. You may also be asked to provide a physiological measure during part of this study. This would entail wearing a pulse monitor on your index finger to provide a physiological measure in response to images. This study will take place in psychology lab at 105 Bond St and will take approximately 60 minutes to complete.

What is Experimental in this Study: None of the procedures or questionnaires used in this study are experimental in nature, in the sense that they have all been used in previous cognitive and social psychology research. From a technical or procedural point of view, part of this study is considered "experimental", because by following the procedure described above, the study examines the impact of one variable (called the "independent variable") on another variables (called the "dependent variable"). More information about the independent and dependent variable will be provided at the end of the session.

Risks or Discomforts: Occasionally people feel uncomfortable when answering questionnaires that ask about their relationship or their attitudes and personal opinions. If any aspect of this study makes you feel uncomfortable, you may choose not to answer certain questions, and you may withdraw from the study at any time.

Benefits of the Study: We anticipate that you will benefit from this study by learning about ways that psychological research can contribute to knowledge on problem-solving and collaboration. When the session is over, we will describe the purpose and hypotheses of the study to you in more detail. Please note that some specific goals and hypotheses of the study will not be revealed to you until the end of your study session. Once we have analyzed the data, you are welcome to ask about the results. However, we cannot guarantee that you will receive any direct benefits from participating in this study. 
Confidentiality: Your responses in this research will remain strictly confidential. We will not put your name or student number on any of the questionnaires or task material. Rather, the questionnaires will be identified by number only. You will be asked to sign only this consent form (if you decide to participate), and it will be filed separately from your questionnaires. The data from this study will be held in a locked office (Dr. Burke's lab), to which only investigators, supervising faculty, and research assistants will have access. The data will be kept for five years, after which time it will be destroyed.

Incentives to Participate: We appreciate the contributions of participants in our research. If you are participating as a PSY102/202 student, you will receive a 1\% course credit toward your research participation mark in your course. If you are a friend of a PSY102/202 student but are not enrolled in either course, you will receive $\$ 10$ as compensation for your participation. If you are a PSY102/202 student and you would prefer to walk through the study (that is, if you would like to observe the research process but not provide any personal data), you will still be given $1 \%$ course credit.

Voluntary Nature of Participation: Participation in this study is voluntary. Your choice of whether or not to participate will not affect your grades or academic status or your future relations with the University. If you decide to participate, you are free to withdraw your consent and to stop your participation at any time without penalty or loss of benefits to which you are allowed. Should you withdraw from the study, you will still be given 1\% course credit (If you are a PSY102/202 student) or $\$ 10$ (if you are not a PSY102/202 student).

Questions about the Study: If you have any questions about the research now, please ask. If you have questions later about the research, you may contact:

Stephanie Marion, smarion@psych.ryerson.ca, 416-979-5000, ext 2190

Dr. Tara Burke, tburke@psych.ryerson.ca, 416-979-5000, ex. 6519

If you have any questions regarding your rights as a human participant in this study, you may contact the Ryerson University Research Ethics Board for information: Toni Fletcher, toni.fletcher@ryerson.ca (416) 979-5000 ext 7112.

Agreement: Your signature below indicates that you have read the information in this agreement and have had a chance to ask any questions you have about the study. Your signature also indicates that you agree to be in the study and have been told that you can change your mind any time during the study and draw from it.

You have been told that by signing this consent agreement, you are not giving up any of your legal rights.

Signature of Participant

Signature of Experimenter

$\overline{\text { Date }}$




\section{Consent Agreement (Strangers condition)}

\section{A Study on the Social Factors that Impact Group Performance on Problem-Solving Tasks}

You are being asked to participate in a research study. Before signing this consent form, it is important that you read the following information and ask any question that you may have.

Investigators:

\begin{tabular}{|c|c|}
\hline $\begin{array}{c}\text { Stephanie Marion, B.Sc., M.A. } \\
\text { Graduate Student } \\
\text { Ryerson University }\end{array}$ & Tara Burke, Ph.D. \\
& $\begin{array}{c}\text { Department of Psychology } \\
\text { Ryerson University }\end{array}$ \\
\hline
\end{tabular}

Purpose of the Study: The primary purpose of this study is to examine how personality characteristics and interpersonal closeness can impact how individuals work together toward a common goal. We are hoping to recruit 132 participants for this study.

Description of the Study: If you decide to participate in the research, you will be asked to do the following: you will be asked to complete a personality questionnaire, a short demographics questionnaire, a series of cognitive problem-solving tasks (e.g., a word-generation task, a word scramble task and a memory task), and a problem-solving strategies questionnaire. Some of these tasks you will do individually and some you will be doing with another participant. Some of your responses to part of this study may require audio-recording. You may also be asked to provide a physiological measure during part of this study. This would entail wearing a pulse monitor on your index finger to provide a physiological measure in response to images. This study will take place in psychology lab at 105 Bond St and will take approximately 60 minutes to complete.

What is Experimental in this Study: None of the procedures or questionnaires used in this study are experimental in nature, in the sense that they have all been used in previous cognitive and social psychology research. From a technical or procedural point of view, part of this study is considered "experimental", because by following the procedure described above, the study examines the impact of one variable (called the "independent variable") on another variables (called the "dependent variable"). More information about the independent and dependent variable will be provided at the end of the session.

Risks or Discomforts: Occasionally people feel uncomfortable when answering questionnaires that ask about their attitudes and personal opinions. If any aspect of this study makes you feel uncomfortable, you may choose not to answer certain questions, and you may withdraw from the study at any time.

Benefits of the Study: We anticipate that you will benefit from this study by learning about ways that psychological research can contribute to knowledge on problem-solving and collaboration. When the session is over, we will describe the purpose and hypotheses of the study to you in more detail. Please note that some specific goals and hypotheses of the study will not be revealed to you until the end of your study session. Once we have analyzed the data, you are welcome to ask about the results. However, we cannot guarantee that you will receive any direct benefits from participating in this study. 
Confidentiality: Your responses in this research will remain strictly confidential. We will not put your name or student number on any of the questionnaires or task material. Rather, the questionnaires will be identified by number only. You will be asked to sign only this consent form (if you decide to participate), and it will be filed separately from your questionnaires. The data from this study will be held in a locked office (Dr. Burke's lab), to which only investigators, supervising faculty, and research assistants will have access. The data will be kept for five years, after which time it will be destroyed.

Incentives to Participate: We appreciate the contributions of participants in our research. You will receive a $1 \%$ course credit toward your research participation mark in your course. If you would prefer to walk through the study (that is, if you would like to observe the research process but not provide any personal data), you will still be given $1 \%$ course credit.

Voluntary Nature of Participation: Participation in this study is voluntary. Your choice of whether or not to participate will not affect your grades or academic status or your future relations with the University. If you decide to participate, you are free to withdraw your consent and to stop your participation at any time without penalty or loss of benefits to which you are allowed. Should you withdraw from the study, you will still be given a $1 \%$ course credit.

Questions about the Study: If you have any questions about the research now, please ask. If you have questions later about the research, you may contact:

Stephanie Marion, smarion@psych.ryerson.ca, 416-979-5000, ext 2190

Dr. Tara Burke, tburke@psych.ryerson.ca, 416-979-5000, ex. 6519

If you have any questions regarding your rights as a human participant in this study, you may contact the Ryerson University Research Ethics Board for information: Toni Fletcher, toni.fletcher@ryerson.ca (416) 979-5000 ext 7112.

Agreement: Your signature below indicates that you have read the information in this agreement and have had a chance to ask any questions you have about the study. Your signature also indicates that you agree to be in the study and have been told that you can change your mind any time during the study and draw from it.

You have been told that by signing this consent agreement, you are not giving up any of your legal rights.

Signature of Participant

Date

Signature of Experimenter

Date 


\section{Demographic and Relationship Questionnaire}

Age:

Gender:

Do you know the other participant in this study?

YES, I know the other participant

NO, I have never met or spoken to this person before

If you have checked YES, please answer the following questions:

1. Please describe the context in which you know the other participant (check all that apply):

- We are friends

We are romantic partners

_ We are related (please specify family relationship: )

We are acquaintances through mutual friends

We are acquaintances through our psychology course or other courses or student groups Other (Please describe:

2. How long have you known each other?

3. How often do you see each other?

per week, OR

per month, OR

per year 
Friendship Qualities Scale (FQS, Bukowski, Hoza, \& Boivin, 1994)

Please provide a rating of how much each of the following statements is true about the friend that is participating in this study with you, on a scale of 1 (absolutely false) to 5 (absolutely true):

$1-$

absolutely

false quite false

neither true nor false quite true

absolutely

true

\begin{tabular}{|l|l|l|l|l|l|}
\hline $\begin{array}{l}\text { 1. My friend and I spend all our free time } \\
\text { together }\end{array}$ & 1 & 2 & 3 & 4 & 5 \\
\hline $\begin{array}{l}\text { 2. If I have a problem at school, at work, or at } \\
\text { home, I can talk to my friend about it }\end{array}$ & 1 & 2 & 3 & 4 & 5 \\
\hline $\begin{array}{l}\text { 3. If other people were bothering me, my friend } \\
\text { would help me }\end{array}$ & 1 & 2 & 3 & 4 & 5 \\
\hline $\begin{array}{l}\text { 4. My friend thinks of fun things for us to do } \\
\text { together }\end{array}$ & 1 & 2 & 3 & 4 & 5 \\
\hline $\begin{array}{l}\text { 5. My friend helps me when I am having trouble } \\
\text { with something }\end{array}$ & 1 & 2 & 3 & 4 & 5 \\
\hline $\begin{array}{l}\text { 6. If my friend had to move away, I would miss } \\
\text { him/her }\end{array}$ & 1 & 2 & 3 & 4 & 5 \\
\hline $\begin{array}{l}\text { 7. When I do a good job at something, my friend } \\
\text { is happy for me }\end{array}$ & 1 & 2 & 3 & 4 & 5 \\
\hline $\begin{array}{l}\text { 8. Sometimes my friend does things for me, or } \\
\text { makes me feel special }\end{array}$ & 1 & 2 & 3 & 4 & 5 \\
\hline $\begin{array}{l}\text { 9. Sometimes I quarrel even violently with my } \\
\text { friend }\end{array}$ & 1 & 2 & 3 & 4 & 5 \\
\hline $\begin{array}{l}\text { 10. My friend would stick up for me if someone } \\
\text { was causing me trouble }\end{array}$ & 1 & 2 & 3 & 4 & 5 \\
\hline $\begin{array}{l}\text { 11. My friend can bug me or annoy me even } \\
\text { though I ask him not to }\end{array}$ & 1 & 2 & 3 & 4 & 5 \\
\hline $\begin{array}{l}\text { 12. If I needed money my friend would loan it to } \\
\text { me }\end{array}$ & 3 & 4 & 5 \\
\hline
\end{tabular}




\begin{tabular}{|c|c|c|c|c|c|}
\hline $\begin{array}{l}\text { 13. After having fought, even violently, with my } \\
\text { friend, if I said sorry to him or her, I think that } \\
\text { he or she would continue to be angry with me }\end{array}$ & 1 & 2 & 3 & 4 & 5 \\
\hline $\begin{array}{l}\text { 14. Sometimes my friend and I just sit around } \\
\text { and talk about things like study, work, and } \\
\text { things we like }\end{array}$ & 1 & 2 & 3 & 4 & 5 \\
\hline 15. My friend would help me if I needed it & 1 & 2 & 3 & 4 & 5 \\
\hline $\begin{array}{l}\text { 16. If there is something bothering me, I can tell } \\
\text { my friend about it even if it is something I } \\
\text { cannot tell to other people }\end{array}$ & 1 & 2 & 3 & 4 & 5 \\
\hline $\begin{array}{l}\text { 17. If my friend or I do something that bothers } \\
\text { the other one of us, we can make up easily }\end{array}$ & 1 & 2 & 3 & 4 & 5 \\
\hline 18. My friend and I can argue a lot & 1 & 2 & 3 & 4 & 5 \\
\hline 19. My friend and I disagree about many things & 1 & 2 & 3 & 4 & 5 \\
\hline $\begin{array}{l}\text { 20. If my friend and I have a violent argument, we } \\
\text { can say "I'm sorry" and everything will be } \\
\text { alright }\end{array}$ & 1 & 2 & 3 & 4 & 5 \\
\hline 21. I feel happy when I am with my friend & 1 & 2 & 3 & 4 & 5 \\
\hline $\begin{array}{l}\text { 22. I think about my friend even when he or she } \\
\text { is not around }\end{array}$ & 1 & 2 & 3 & 4 & 5 \\
\hline
\end{tabular}




\section{Right-Wing Authoritarianism Scale (Altemeyer, 1981)}

\section{Personality Questionnaire - Match check}

Please complete the following brief personality/opinions questionnaire. Indicate the degree to which you agree or disagree with each statement by circling the appropriate number:

\section{$\begin{array}{lllllllll}-4 & -3 & -2 & -1 & 0 & +1 & +2 & +3 & +4\end{array}$ Very strongly disagree Neutral Very strongly agree}

1. Our country desperately needs a mighty leader who will do what has to be done to destroy the radical new ways and sinfulness that are ruining us.

$\begin{array}{lllllllll}-4 & -3 & -2 & -1 & 0 & +1 & +2 & +3 & +4\end{array}$

2. Gays and lesbians are just as healthy and moral as anybody else.
$-4$
$-3$
$-2-1$
$0+1$
$+2+3+4$

3. It is always better to trust the judgment of the proper authorities in government and religion, than to listen to the noisy rabble-rousers in our society who are trying to create doubt in people's minds.

$\begin{array}{lllllllll}-4 & -3 & -2 & -1 & 0 & +1 & +2 & +3 & +4\end{array}$

4. Atheists and others who have rebelled against the established religions are no doubt every bit as good and virtuous as those who attend church regularly.

$\begin{array}{lllllllll}-4 & -3 & -2 & -1 & 0 & +1 & +2 & +3 & +4\end{array}$

5. The only way our country can get through the crisis ahead is to get back to our traditional values, put some tough leaders in power, and silence the troublemakers spreading bad ideas.

$\begin{array}{llllllll}-4 & -3 & -2 & -1 & 0 & +1 & +2 & +3\end{array}$

6. There is absolutely nothing wrong with nudist camps.

$\begin{array}{lllllllll}-4 & -3 & -2 & -1 & 0 & +1 & +2 & +3 & +4\end{array}$

7. Our country needs free thinkers who will have the courage to defy traditional ways, even if this upsets many people.

$\begin{array}{lllllllll}-4 & -3 & -2 & -1 & 0 & +1 & +2 & +3 & +4\end{array}$

8. Our country will be destroyed someday if we do not smash the perversions eating away at our moral fibre and traditional beliefs.

$\begin{array}{lllllllll}-4 & -3 & -2 & -1 & 0 & +1 & +2 & +3 & +4\end{array}$

9. Everyone should have their own lifestyle, religious beliefs, and sexual preferences, even if it makes them different from everyone else.

$\begin{array}{lllllllll}-4 & -3 & -2 & -1 & 0 & +1 & +2 & +3 & +4\end{array}$


10. The "old-fashioned ways" and "old-fashioned values" still show the best way to live.

$\begin{array}{lllllllll}-4 & -3 & -2 & -1 & 0 & +1 & +2 & +3 & +4\end{array}$

11. You have to admire those who challenged the law and the majority's view by protesting for abortion rights, for animal rights, or to abolish school prayer.

$\begin{array}{lllllllll}-4 & -3 & -2 & -1 & 0 & +1 & +2 & +3 & +4\end{array}$

12. What our country really needs is a strong, determined leader who will crush evil, and take us back to our true path.

$\begin{array}{lllllllll}-4 & -3 & -2 & -1 & 0 & +1 & +2 & +3 & +4\end{array}$

13. Some of the best people in our country are those who are challenging our government, criticizing religion, and ignoring the "normal way things are supposed to be done."

$\begin{array}{lllllllll}-4 & -3 & -2 & -1 & 0 & +1 & +2 & +3 & +4\end{array}$

14. God's laws about abortion, pornography, and marriage must be strictly followed before it is too late, and those who break them must be strongly punished.

$\begin{array}{llllllll}-4 & -3 & -2 & -1 & 0 & +1 & +2 & +3\end{array}$

15. There are many radical, immoral people in our country today, who are trying to ruin it for their godless purposes, who the authorities should put out of action.

$\begin{array}{lllllllll}-4 & -3 & -2 & -1 & 0 & +1 & +2 & +3 & +4\end{array}$

16. A "woman's place" should be wherever she wants to be. The days when women are submissive to their husbands and social conventions belong strictly in the past.

$\begin{array}{lllllllll}-4 & -3 & -2 & -1 & 0 & +1 & +2 & +3 & +4\end{array}$

17. Our country will be great if we honour the ways of our forefathers, do what the authorities tell us to do, and get rid of the "rotten apples" who are ruining everything.

$\begin{array}{lllllllll}-4 & -3 & -2 & -1 & 0 & +1 & +2 & +3 & +4\end{array}$

18. There is no "ONE right way" to live life; everybody has to create their own way.

$\begin{array}{llllllll}-4 & -3 & -2 & -1 & 0 & +1 & +2 & +3\end{array}$

19. Homosexuals and feminists should be praised for being brave enough to defy "traditional family values."

$\begin{array}{lllllllll}-4 & -3 & -2 & -1 & 0 & +1 & +2 & +3 & +4\end{array}$

20. This country would work a lot better if certain groups of troublemakers would just shut up and accept their group's traditional place in society.

$\begin{array}{lllllllll}-4 & -3 & -2 & -1 & 0 & +1 & +2 & +3 & +4\end{array}$

21. It would be best for everyone if the proper authorities censored magazines so that people could not get their hands on trashy and disgusting material.

$\begin{array}{llllllll}-4 & -3 & -2 & -1 & 0 & +1 & +2 & +3\end{array}+4$ 
22. There is nothing wrong with premarital sexual intercourse.
$-4$
$-2-1$
$0+1+2+3+4$

23. People should pay less attention to the Bible and the other forms of religious guidance, and instead develop their own personal standards of what is moral and immoral.

$\begin{array}{lllllllll}-4 & -3 & -2 & -1 & 0 & +1 & +2 & +3 & +4\end{array}$

24. What our country needs most is discipline, with everyone following our leaders in unity.

$\begin{array}{lllllllll}-4 & -3 & -2 & -1 & 0 & +1 & +2 & +3 & +4\end{array}$

25. A lot of our rules regarding modesty and sexual behaviour are just customs that are not necessarily any better or holier than those which other people follow.

$\begin{array}{lllllllll}-4 & -3 & -2 & -1 & 0 & +1 & +2 & +3 & +4\end{array}$

26. The facts on crime, sexual immorality, and the recent public disorders all show we have to crack down harder on deviant groups and troublemakers if we are going to save our moral standards and preserve law and order.

$\begin{array}{lllllllll}-4 & -3 & -2 & -1 & 0 & +1 & +2 & +3 & +4\end{array}$

27. It's better to have trashy magazines and radical pamphlets in our communities than to let the government have the power to censor them.

$\begin{array}{lllllllll}-4 & -3 & -2 & -1 & 0 & +1 & +2 & +3 & +4\end{array}$

28. The situation in our country is getting so serious, the strongest methods would be justified if they eliminated the troublemakers and got us back on our true path.

$\begin{array}{lllllllll}-4 & -3 & -2 & -1 & 0 & +1 & +2 & +3 & +4\end{array}$

29. It is wonderful that young people today have greater freedom to protest against things they don't like, and to make their own "rules" to govern their behaviour.

$\begin{array}{lllllllll}-4 & -3 & -2 & -1 & 0 & +1 & +2 & +3 & +4\end{array}$

30. Once the government leaders give us the "go-ahead," it will be the duty of every patriotic citizen to help stomp out the rot that is poisoning our country from within.

$\begin{array}{llllllll}-4 & -3 & -2 & -1 & 0 & +1 & +2 & +3\end{array}$ 


\section{Consent Form for Alibi Provider in Study 3}

\section{Consent Agreement \\ The Effect of Friendship on Corroboration of False Alibis}

You are being asked to participate in a research study that is different from the one for which you originally provided consent. The study entitled "A Study on the Social Factors that Impact Group Performance on Problem-Solving Tasks" was a cover story for what we are really interested in studying. The information below details the true purpose of the study. Before signing this consent form, it is important that you read through this information and discuss any concerns or questions that you may have with the experimenter. You may ask as many questions as necessary to be sure that you understand what the study entails.

Investigators:

\begin{tabular}{|c|c|}
\hline $\begin{array}{c}\text { Stephanie Marion, B.Sc., M.A. } \\
\text { Graduate Student } \\
\text { Ryerson University }\end{array}$ & Tara Burke, Ph.D. \\
& $\begin{array}{c}\text { Department of Psychology } \\
\text { Ryerson University }\end{array}$ \\
\hline
\end{tabular}

Purpose of the Study: An "alibi" is a story given by someone that details what he or she was doing at a specific time. The primary purpose of this study is to determine whether individuals are more likely to corroborate an alibi that they know is false when the alibi is provided by a friend than when it is provided by a stranger. If you are participating in this study with a friend, you are in the experimental condition, and if you are participating with a stranger, you are in the control condition.

Description of the Study: If you decide to participate in the research, you will be asked to play the role of a confederate (i.e., a helper to the experimenter). This will entail continuing on with the completion of the cognitive tasks as the original consent form outlines, but also to follow certain directions from the experimenter as you are doing so. Specifically, upon your return to the study room to complete the collaborative tasks, you will be asked to leave briefly at a predetermined time, under the guise of needing to use the washroom. Then, towards the end of the study, the experimenter will pretend that a theft occurred in an office nearby and will ask you and the other participant whether you know anything about it. At this point, you will be asked to provide a "false alibi" by saying that both of you were in the study room for the entire duration of the study. A few minutes later, when the other participant and you are in different rooms, the other participant will be asked if both of you were indeed in the room the whole time. Of course, for this study to work, you will be asked to pretend that you are unaware of the true purpose of the study. To ensure that you do not accidently say something to reveal the true purpose of the study, there will be a voiceactivated audio-recorder in the study room, if you provide consent to being recorded (see below). Please note that the true purpose of this study will be fully explained to the other participant at the end of the study, and their consent to keep their data in the study will be asked of them at that time.

What is Experimental in this Study: None of the procedures or questionnaires used in this study are experimental in nature, in the sense that they have all been used in previous cognitive and social psychology research. From a technical or procedural point of view, part of this study is considered "experimental", because by following the procedure described above, the study examines the impact of one variable (called the "independent variable") on another variables (called the 
"dependent variable"). More information about the independent and dependent variable will be provided at the end of the session.

Risks or Discomforts: It is possible that you may feel slightly anxious about not telling the other participant about the true purpose of the study, or about their "acting" part in the study (e.g., saying that you need to go to the washroom, and telling the false alibi). The number and duration of these white lies, necessary for the cover story, will be kept at a minimum and the other participant will be fully debriefed immediately at the end of the study. Please know that we very much appreciate your participation, and that we know that you are not a professional actor, and that we are asking you to behave as normally as possible. If any aspect of this study makes you feel uncomfortable, you may choose not to withdraw from the study at any time, without any loss of benefits to which you are allowed.

Benefits of the Study: We anticipate that you will benefit from this study by learning about different psychological research methods and ways that psychological research can contribute to knowledge on alibi corroboration and other topics that relate to psychology and the law. When the session is over, we will describe the hypotheses of the study to you in more detail. Once we have analyzed the data, you are welcome to ask about the results. However, we cannot guarantee that you will receive any direct benefits from participating in this study.

Confidentiality: Your responses in this research will remain strictly confidential. We will not put your name or student number on any of the questionnaires or task material. Rather, the questionnaires will be identified by number only. You will be asked to sign only this consent form (if you decide to participate), and it will be filed separately from your questionnaires. The audiorecording (if you consent to it) used to ensure the integrity of the study results will be reviewed once by the experimenter at the end of the day and will be destroyed immediately. It will not be transcribed and the file will not be kept. The remainder of the data (questionnaires and response sheets) from this study will be held in a locked office (Dr. Burke's lab), to which only investigators, supervising faculty, and research assistants will have access. The data will be kept for five years, after which time it will be destroyed.

Incentives to Participate: Compensation for taking part in this study will be as outlined in the original consent form: You will receive a 1\% course credit toward your research participation mark in your course (if you are a PSY102/202 student) or \$10 (if you are not a PSY102/202 student).

Voluntary Nature of Participation: Participation in this study is voluntary. Your choice of whether or not to participate will not affect your grades or academic status or your future relations with the University. If you decide to participate, you are free to withdraw your consent and to stop your participation at any time without penalty or loss of benefits to which you are allowed. Should you withdraw from the study, you will still be given a $1 \%$ course credit (or $\$ 10$ ).

Questions about the Study: If you have any questions about the research now, please ask. If you have questions later about the research, you may contact:

Stephanie Marion, smarion@psych.ryerson.ca, 416-979-5000, ext 2190

Dr. Tara Burke, tburke@psych.ryerson.ca, 416-979-5000, ex. 6519

If you have any questions regarding your rights as a human participant in this study, you may contact the Ryerson University Research Ethics Board for information: Toni Fletcher, toni.fletcher@ryerson.ca (416) 979-5000 ext 7112. 
Agreement: Your signature below indicates that you have read the information in this agreement and have had a chance to ask any questions you have about the study. Your signature also indicates that you agree to be in the study and have been told that you can change your mind any time during the study and draw from it.

You have been told that by signing this consent agreement, you are not giving up any of your legal rights.

Name of Participant (please print)

$\overline{\text { Signature of Participant }}$

Date

Signature of Experimenter

Date

Agreement to being audio-recorded: Your signature below indicates that you agree to being audiorecorded during this study, and that you understand that this recording will be reviewed by the experimenter and destroyed by the end of today. If you change your mind later and decide to withdraw consent, please let the experimenter know before the end of the study, and the audiorecording will be destroyed without being reviewed.

$\overline{\text { Signature of Participant }}$

Date 
The other participant is currently completing a series of questionnaires and will also complete some cognitive tasks (a word search task and an anagram task). The other participant also believes that you are currently completing the same questionnaires and tasks.

When I bring you back to the testing room, I will give you and the other participant instructions for three tasks: the first and third tasks you will do collaboratively, but the $2^{\text {nd }}$ task I will assign the other participant to complete (pretending that he/she was chosen randomly). At the beginning of this individual task (an image-rating task), I will ask you to excuse yourself to go to the washroom. You can say something like "I need to go to the washroom, I'll be right back", and leave the room, closing the door behind you. You can then come back to this room for three minutes (I will be here to time it), then simply reenter the testing room, closing the door behind you. You can then complete the third task with the other participant.

At the end of the third task, I will enter the testing room and inform both of you that a theft occurred during the time you were completing the three tasks (i.e., that someone stole money from the next-door office), and I will ask if anyone has seen anything. I will ask that you immediately answer me, as naturally as possible, "No, we were both here the whole time".

I will then proceed with the study and separate you and the other participant again for another individual part of the study.

Remember, there are two things that you are asked to do:

1) leave the room under pretext that you need to use the washroom, when he/she starts the image-rating task, and

2) answer "no, we were both here the whole time" when I ask if anyone has seen anything suspicious.

Other than those two things, please simply act as normally as possible, and simply follow the instructions for the collaborative tasks. Please do not say or signal anything to the other participant to let him/her know of these instructions or of the true purpose of the study. A voice-activated audio-recording device has been installed to make sure nothing is said, and this recording file will be destroyed after it is reviewed, by the end of today.

Do you have any questions?

Are you comfortable with what you have been asked to do? 


\section{Debriefing - Alibi Provider}

Thank you for participating in this experiment. As was explained earlier, the aim of this study was to examine how interpersonal closeness can impact alibi corroboration. An alibi is a type of statement that a person suspected of a crime provides to police to indicate that he or she could not have committed the crime due to being elsewhere from where the crime was committed. Sometimes such statements are corroborated (or confirmed) by another witness. In this study, we want to know whether people who are friends are more likely than strangers to support one another's alibi, even if the alibi provided is false.

Courts seem to think that people lie for one another, and especially those who are close to one another. This assumption has important implications for the believability of a defendant's alibi in court. This study examines the validity of this assumption. Depending on the condition in which you were assigned, the other participant in this study was either a friend (the experimental condition) or another student that you did not know (the control condition). Thus, the relationship between you and the other participant is the independent variables in this study. During the first part of the study, while the other participant was completing the personality and demographic questionnaires, you were recruited to be a confederate in this study - someone who is aware of the experiment and is helping out the experimenter.

We hypothesized that friends will be more likely to corroborate the other participant's alibi than strangers. That is, they would be more likely to agree that the confederate remained in the testing room during the entire session (the dependent variable).

The purpose of the study is to understand how personal affiliations to others influence the likelihood of supporting a statement that may not be completely true. The results will contribute to our theoretical understanding of alibi corroboration and perception, and will allow us to make recommendations to the police regarding their ability to assess the credibility of alibis. The results also have implications for police investigative processes and for determining factors involved in wrongful convictions.

In the past, participants who have taken part in this study and who have been given the opportunity to support a false alibi (or refute it) has done so for many reasons. For example, many participants who told the experimenter the truth by saying that the other participant left to use the washroom said they did so because they did not think that the other person was guilty of the theft and that going to the washroom was a very innocent thing to do. Other participants who did not tell the truth and told the experimenter that the other participant had not left the room said they did so because they did not think the other person was guilty and didn't want to arouse undue suspicion. Thus, in general people chose to both corroborate or refute false alibis for very pro-social reasons.

We would like to remind you that your results are completely confidential and that all results are published anonymously as a group. However, if you feel uncomfortable about any aspect of the study, you are free to request to have your data withdrawn from the sample. Also, if you know of others who will be participating in this experiment, please refrain from discussing it with them. We do not want our future participants to be aware of the procedures and expected findings. 
We would like to thank you again for your participation in our study. Please retain this form for future reference and feel free to contact the student experimenter or Professor Burke if you have any further questions or concerns, or if you would like any information about the results once it is completed:

\begin{tabular}{|c|c|}
\hline Stephanie Marion, B.Sc., M.A. & Tara Burke, Ph.D. \\
Graduate Student & Department of Psychology \\
Ryerson University & Ryerson University \\
smarion@psych.ryerson.ca & tburke@psych.ryerson.ca \\
416-979-5000, ext 2190 & $416-979-5000$, ex. 6519 \\
\hline
\end{tabular}




\section{Debriefing - Alibi Witness}

Thank you for participating in this experiment. As you may recall, you were told that the aim of this study was to examine how personality characteristics and interpersonal closeness can impact collaboration on cognitive tasks. However, the actual purpose of this research is to examine the impact of interpersonal closeness on alibi corroboration. An alibi is a type of statement that a person suspected of a crime provides to police to indicate that he or she could not have committed the crime due to being elsewhere from where the crime was committed. Sometimes such statements are corroborated (or confirmed) by another witness. In this study, we want to know whether people who are friends are more likely than strangers to support one another's alibi, even if the alibi provided is false.

Courts seem to think that people lie for one another, and especially those who are close to one another. This assumption has important implications for the believability of a defendant's alibi in court. This study examines the validity of this assumption. Depending on the condition in which you were assigned, the other participant in this study was either a friend (the experimental condition) or another student that you did not know (the control condition). Thus, the relationship between you and the other participant is the independent variables in this study. During the first part of the study, while you were completing the personality and demographic questionnaires, we recruited the other participant to be a confederate in this study - someone who is aware of the experiment and is helping out the experimenter. It was during this time that they were instructed to leave the testing room to go to the washroom, at to provide their "alibi" that they were in the testing room during the entire time of the tasks. Thus, please be aware that no theft occurred, and that the other participant had nothing to do with a theft and is not suspected of stealing anything.

We hypothesized that friends will be more likely to corroborate the other participant's alibi than strangers. That is, they would be more likely to agree that the confederate remained in the testing room during the entire session (the dependent variable).

The purpose of the study is to understand how personal affiliations to others influence the likelihood of supporting a statement that may not be completely true. The results will contribute to our theoretical understanding of alibi corroboration and perception, and will allow us to make recommendations to the police regarding their ability to assess the credibility of alibis. The results also have implications for police investigative processes and for determining factors involved in wrongful convictions.

As I'm sure you noticed there was some deception in this experiment - that is, some aspects of the studies were not as originally described. In particular, the information you received about the purpose of the study was not fully explained to you at the start. Our main interests were in how you would respond when asked whether the other participant had been in the study room during the entire session. We hope that you understand the reasons why the real purpose of the experiment could not be revealed to you until now. We couldn't have obtained your unbiased reactions to the situations if you had known exactly what we were interested in. As a result, we had to set up somewhat contrived situations. In addition, in order to ensure the integrity of the study results, there was a voice-activated audio-recording device in the testing room. The 
purpose of this is to ensure that the other participant did not divulge the true purpose of the study, or any part of it, during the study. This audio recording, if you agree to it, will be reviewed by the experimenter and then destroyed by the end of today. It will not be transcribed, nor will copies of the file be kept.

We would like to remind you that your results are completely confidential and that all results are published anonymously as a group. However, if you feel uncomfortable about any aspect of the study, you are free to request to have your data withdrawn from the sample. Also, if you know of others who will be participating in this experiment, please refrain from discussing it with them. We do not want our future participants to be aware of the procedures and expected findings.

We would like to thank you again for your participation in our study. Please retain this form for future reference and feel free to contact the student experimenter or Professor Burke if you have any further questions or concerns, or if you would like any information about the results once it is completed:

\begin{tabular}{|c|c|}
\hline $\begin{array}{c}\text { Stephanie Marion, B.Sc., M.A. } \\
\text { Graduate Student }\end{array}$ & Tara Burke, Ph.D. \\
Ryerson University & Department of Psychology \\
smarion@psych.ryerson.ca & Ryerson University \\
416-979-5000, ext 2190 & tburke@psych.ryerson.ca \\
\hline
\end{tabular}


Agreement: Your signature below indicates that you have read the information in this debriefing form and have had a chance to ask any questions you have about the study. Your signature also indicates that you agree to allow the experimenter to retain the data collected in the study now that you have full knowledge of the purpose of this study.

You have been told that by signing this consent agreement, you are not giving up any of your legal rights.

Name of Participant (please print)

Signature of Participant

Date

Signature of Investigator

Date

Agreement to being audio-recorded: Your signature below indicates that you allow the experimenter to review the audio-recording taken during the study. You also understand that the audio file will be destroyed by the end of today. If you do not consent to the experimenter reviewing the audio-recording, the file will be destroyed immediately, in your presence, without it being reviewed.

Signature of Participant

Date 


\section{APPENDIX G}

Participants' explanations for rejection and corroboration of the alibi, by relationship condition. Note that many participants provided more than one answer.

\begin{tabular}{|c|c|c|c|c|c|c|c|}
\hline \multirow[b]{3}{*}{ Response categories } & \multicolumn{3}{|c|}{$\begin{array}{l}\text { Participants who did not } \\
\text { corroborate the alibi }(n=48)\end{array}$} & \multicolumn{3}{|c|}{$\begin{array}{c}\text { Participants who corroborated } \\
\text { the alibi }(n=20)\end{array}$} & \multirow[t]{3}{*}{ Total } \\
\hline & \multicolumn{2}{|c|}{$\begin{array}{l}\text { Relationship } \\
\text { Condition }\end{array}$} & \multirow{2}{*}{ Total } & \multicolumn{2}{|c|}{$\begin{array}{l}\text { Relationship } \\
\text { Condition }\end{array}$} & \multirow{2}{*}{ Total } & \\
\hline & $\begin{array}{l}\text { Strangers } \\
(n=28)\end{array}$ & $\begin{array}{l}\text { Friends } \\
(n=20)\end{array}$ & & $\begin{array}{l}\text { Strangers } \\
(n=6)\end{array}$ & $\begin{array}{l}\text { Friends } \\
(n=14)\end{array}$ & & \\
\hline \multicolumn{8}{|l|}{ Participant-centered } \\
\hline Morality-based response & 18 & 12 & 30 & -- & -- & -- & 30 \\
\hline $\begin{array}{l}\text { Avoiding confrontation } \\
\text { or involvement }\end{array}$ & 5 & 7 & 12 & -- & 1 & 1 & 13 \\
\hline Memory lapse & -- & -- & -- & 5 & 5 & 10 & 10 \\
\hline Caught off guard & -- & -- & -- & -- & 2 & 2 & 2 \\
\hline \multicolumn{8}{|l|}{ Confederate-centered } \\
\hline $\begin{array}{l}\text { Protection of confederate } \\
\text { or relationship }\end{array}$ & -- & -- & -- & 1 & 3 & 4 & 4 \\
\hline $\begin{array}{l}\text { Lack of trust in } \\
\text { confederate }\end{array}$ & 8 & 2 & 10 & -- & 1 & 1 & 11 \\
\hline Trust in the confederate & 4 & 11 & 15 & 4 & 12 & 16 & 31 \\
\hline \multicolumn{8}{|l|}{ Situation-centered } \\
\hline Compliance & 5 & 3 & 8 & -- & -- & -- & 8 \\
\hline Irrelevance/innocuous & -- & -- & -- & -- & 1 & -- & 1 \\
\hline
\end{tabular}




\section{References}

Allison, M., \& Brimacombe, C. A. E. (2010). Alibi believability: The effect of prior convictions and judicial instructions. Journal of Applied Social Psychology, 40(5), 1054-1084.

Allison, M., Mathews, K. R., \& Michael, S. W. (2012). Alibi believability: The impact of salacious alibi activities. Social Behavior and Personality, 40(4), 605-612.

Allison, M., Michael, S. W., Mathews, K. R., \& Overman, A. A. (2011). Brief report: Narrative qualities and perceptions of generated alibis. North American Journal of Psychology, $13(3), 359-365$.

Altemeyer, B. (1981) Right-wing authoritarianism. Winnipeg: University of Manitoba Press.

Berkowitz, L. (1972). Social norms, feelings, and other factors affecting helping and altruism. In L. Berkowitz (Ed.), Advances in experimental social psychology (Vol. 6). New York, NY: Academic Press.

Berkowitz, L. \& Daniels, L. R. (1963). Responsibility and dependency. Journal of Abnormal and Social Psychology, 66(5), 429-436.

Berkowitz, L. \& Daniels, L. R. (1964). Affecting the salience of the social responsibility norm: Effects of past help on the response to dependency relationships. Journal of Abnormal and Social Psychology, 68, 275-281.

Berscheid, E. \& Walster, E. (1978). Interpersonal attraction. Reading, Mass.: Addison, Wesley.

Bukowski, W. M., Hoza, B., \& Boivin, M. (1994). Measuring friendship quality during pre- and early adolescence: The development and psychometric properties of the Friendship Qualities Scale. Journal of Social and Personal Relationships, 11, 471-484. 
Burger, J. M., Messian, N., Patel, S., del Prado, A., \& Anderson, C. (2004). What a coincidence! The effects of incidental similarity on compliance. Personality and Social Psychology Bulletin, 30(1), 35-43.

Burger, J.M., Soroka, S., Gonzago, K., Murphy, E., \& Somervell, E. (2001). The effect of fleeting attraction on compliance to requests. Personality and Social Psychology Bulletin, 27(12), 1578-1586. doi: 10.1177/01461672012712002

Burke, T. M., \& Marion, S. B. (2012). Alibi witnesses. In B. L. Cutler (Ed.), Conviction of the innocent: Lessons from psychological research (pp.239-256). Washington, DC: American Psychological Association.

Burke, T.M. \& Turtle. J.W. (2003). Alibi evidence in criminal investigations and trials: Psychological and legal factors. Canadian Journal of Police and Security Services, 1(3), 286-294.

Burke, T.M., Turtle, J.W., \& Olson, E. (2007). A psychological approach to the study of alibis. Book chapter in The Handbook of Eyewitness Psychology, Toglia, M., Read, J.D., Ross, D., Lindsay, R.C.L. (editors): Erlbaum.

Byrne, D. (1971). The Attraction Paradigm. New York: Academic Press.

Byrne, D. (1997). An overview (and underview) of research and theory within the attraction paradigm. Journal of Social and Personal Relationships, 14, 417-431. doi: $10.1177 / 0265407597143008$

Cialdini, R. B., \& Goldstein, N. J. (2004). Social influence: Compliance and conformity. Annual Review of Psychology, 55, 591-621. doi: 10.1146/annurev.psych. 55.090902.142015 
Cialdini, R. B., \&Trost, M. R. (1998). Social influence: Social norms, conformity, and compliance. In D. T. Gilbert, S. T. Fiske, \& G. Lindzey (Ed.), Handbook of social psychology, Vol. 2 (4th ed., pp. 151-192). Boston: McGraw-Hill.

Connors, E. T., Lundregan, T., Miller, N., \& McEwen, T. (1996). Convicted by juries, exonerated by science: Case studies in the use of DNA evidence to establish innocence after trial. Washington, DC: U.S. Department of Justice, National Institute of Justice.

Crawford, J. R., \& Henry, J. D. (2004). The positive and negative affect schedule (PANAS): Construct validity, measurement properties and normative data in a large non-clinical sample. The British Journal of Clinical Psychology, 43, 245-65.

Culhane, S.E. \& Hosch, H.M. (2004). An alibi witness' influence on mock jurors' verdicts. Journal of Applied Social Psychology, 34(8), 1604-1616.

Culhane, S. E., \& Hosch, H. M. (2012). Changed alibis: Current law enforcement, future law enforcement, and layperson reactions. Criminal Justice and Behavior, 39(7), 958-977.

Culhane, S.E., Hosch, H.M., \& Kehn, A. (2008). Alibi generation: Data from U.S. Hispanics and U.S. non-Hispanic Whites. Journal of Ethnicity in Criminal Justice, 6(3), 177-199.

Culhane, S. E., Kehn, A., Horgan, A. J., Meissner, C. A., Hosch, H. M., \& Wodahl, E. J. (2013). Generation and detection of true and false alibi statements. Psychiatry, Psychology, and Law, 20(4), 619-638. doi: 10.1080/13218719.2012.729018

Dahl, L.C., Brimacombe, C.A.E., \& Lindsay, D.S. (2009). Investigating investigators: How presentation order influences participant-investigators' interpretations of eyewitness identification and alibi evidence. Law and Human Behavior, 33(5), 368-380. 
Dahl, L. C., \& Price, H. L. (2012). "He couldn't have done it, he was with me!”: The impact of alibi witness age and relationship. Applied Cognitive Psychology, 26(3), 475-481. doi: 10.1002/acp. 2821

Daniels, L. R., \& Berkowitz, L. (1963). Liking and response to dependency relationships. Human Relations, 16, 141-148.

Darley, J. M., \& Latané, B. (1968). Bystander intervention in emergencies: Diffusion of responsibility. Journal of Personality and Social Psychology, 8(4), 377-383.

Davis, J. N., \& Daly, M. (1997). Evolutionary theory and the human family. The Quarterly Review of Biology, 72(4), 407-435. doi:

DePaulo, B. M., Ansfield, M. E., Kirkendol, S. E., \& Boden, J. M. (2004). Serious lies. Basic and Applied Social Psychology, 26 (2/3), 147-167.

DePaulo, B. M. \& Kashy, D. A. (1998). Everyday lies in close and casual relationships. Journal of Personality and Social Psychology, 74(1), 63-79.

DePaulo, B. M., Kashy, D. A., Kirkendol, S. E., Wyer, M. M., \& Epstein, J. A. (1996). Lying in everyday life. Journal of Personality and Social Psychology, 70I, 979-995.

Dieckman, A. B. \& Eagly, A. H. (2000). Stereotypes as dynamic constructs: Women and men of the past, present, and future. Personality and Social Psychology Bulletin, 26(10), 11711188.

Downey, R. G., \& King, C. V. (1998). Missing data in Likert ratings : A comparison of replacement methods. The Journal of General Psychology, 125(2), 175-191.

Dysart, J. E., \& Strange, D. (2012). Beliefs about alibis and alibi investigations: A survey of law enforcement. Psychology, Crime \& Law, 18(1), 11-25. 
Eagly, A. H., \& Crowley, M. (1986). Gender and helping behaviour: A meta-analytic review of the social psychological literature. Psychology Bulletin, 100, 283-308.

Ennis, E., Vrij, A., \& Chance, C. (2008). Individual differences and lying in everyday life. Journal of Social and Personal Relationships, 25(1), 105-118. doi: $10.1177 / 0265407507086808$

Faul, F., Erdfelder, E., Lang, A.-G., \& Buchner, A. (2007). G*Power 3: A flexible statistical power analysis program for the social, behavioral, and biomedical sciences. Behavior Research Methods, 39, 175-191.

Fu, G., Evans, A. D., Wang, L., \& Lee, K. (2008). Lying in the name of the collective good: A developmental study. Developmental Science, 11(4), 495-503. doi:10.1111/j.14677687.2008.00695.x

Garrett, B. L. (2008). Judging innocence. Columbia Law Review, 108, 56-141.

Goranson, R. E., \& Berkowitz, L. (1966). Reciprocity and responsibility reactions to prior help. Journal of Personality and Social Psychology, 3, 227-232.

Gosling, S. D., Rentfrow, P. J., \& Swann, W. B., Jr. (2003). A Very Brief Measure of the Big Five Personality Domains. Journal of Research in Personality, 37, 504-528. doi: 10.1016/S0092-6566(03)00046-1

Gouldner, A. W. (1960). The norm of reciprocity: A preliminary statement. American Sociological Review, 25, 161-178.

Granhag, P., Strömwall, L. A., \& Jonsson, A. (2003). Partners in crime: How liars in collusion betray themselves. Journal of Applied Social Psychology, 33(4), 848-868.

Greenglass, E. R. (1972). Effects of age and prior help on "altruistic lying". The Journal of Genetic Psychology: Research and Theory on Human Development, 121(2), 303-313. 
Hamilton, W. D. (1964). The genetical evolution of social behavior. Journal of Theoretical Biology, 7(1), 1-52. doi:10.1016/0022-5193(64)90038-4

Harris, D. B. A. (1957). A scale for measuring attitudes of social responsibility in children. Journal of Abnormal and Social Psychology, 55, 322-326.

Hawthorne, G., \& Elliott, P. (2005). Imputing cross-sectional missing data: comparison of common techniques. Australian and New Zealand Journal of Psychiary, 39, 583-590.

Hosch, H.M., Culhane, S.E., Jolly, K.W., Chavez, R.M., \& Shaw, L.H. (2011). Effects of an alibi witness's relationship to the defendant on mock jurors' judgments. Law and Human Behavior, 35(2), 127-142.

Huey, L. \& Quirouette, M. (2010). ‘Any girl can call the cops, no problem': The influence of gender on support for the decision to report criminal victimization within homeless communities. The British Journal of Criminology, 50(2), 278-295. doi: 10.1093/bjc/azp078

Insko, C.A. \& Wilson, M. (1977). Interpersonal attraction as a function of social interaction. Journal of Personality and Social Psychology, 35(12), 903-911.

Jung, S., Allison, M., \& Bohn, L. (2013). Legal decision-making on crimes involving an alibi. Applied Psychology in Criminal Justice, 9(1), 45-58.

Kashy, D. A., \& DePaulo, B. M. (1996). Who lies? Journal of Personality and Social Psychology, 70, 1037-1051.

Kassin, S. M., Goldstein, C. C., \& Savitsky, K. (2003). Behavioral confirmation in the interrogation room: On the dangers of presuming guilt. Law and Human Behavior, 27(2), 187-203. 
Kraus, S. J. (1995). Attitudes and the prediction of behaviour: A meta-analysis of the empirical literature. Personality and Social Psychology Bulletin, 21, 58-75.

Lindsay, R.C.L., Lim, R., Marando, L., \& Cully, D. (1986). Mock juror evaluations of eyewitness testimony: A test of metamemory hypotheses. Journal of Applied Social Psychology, 15, 447-459.

Mack, A. \& Rock, I. (1998). Inattentional Blindness. Cambridge, MA: MIT Press.

Madsen, E. A., Tunney, R. J., Fieldman, G., Plotkin, H. C., Dunbar, R. I. M., Richardson, J.-M., \& McFarland, D. (2007). Kinship and altruism: A cross-cultural experimental study. British Journal of Psychology, 98, 339-359. doi: 10.1348/000712606X129213

MacLin, M. K., \& Herrera, V. (2006). The criminal stereotype. North American Journal of Psychology, (8)2, 197-207.

Marion, S. B. \& Burke, T. M. (2013). False alibi corroboration: Witnesses lie for suspects who seem innocent, whether they like them or not. Law and Human Behavior, 37(2), 136-143.

Maeder, E. M. \& Dempsey, J. L. (2013). A likely story? The influence of type of alibi and defendant gender on juror decision-making, Psychiatry, Psychology and Law, doi:10.1080/13218719.2012.727066

Nunnally, J. C. (1978). Psychometric theory (2nd ed.). New York: McGraw-Hill.

Olson, E. A., \& Charman, S. D. (2012). 'But can you prove it?'-Examining the quality of innocent suspects' alibis. Psychology, Crime \& Law, 18(5), 453-471.

Olson, E.A., \& Wells, G.L. (2004). What makes a good alibi? A proposed taxonomy. Law and Human Behavior, 28, 157-176.

Olson, E. A., \& Wells, G. L. (2010). The alibi-generation effect: Alibi-generation experience influences alibi evaluation. Legal and Criminological Psychology, 17(1), 151-164. doi: 10.1111/j.2044-8333.2010.02003.x 
Ponti, L., Guarnieri, S., Smorti, A., \& Tani, F. (2010). A measure for the study of friendship and romantic relationship quality from adolescence to early-adulthood. The Open Psychology Journal, 3, 76-87.

Rachlin, H., \& Jones, B. A. (2008). Altruism among relatives and non-relatives. Behavioural Processes, 79(2), 120-123.

Regan, D. T. (1971). Effects of a favor and liking on compliance. Journal of Experimental Social Psychology, 7(6), 627-639. doi: 10.1016/0022-1031(71)90025-4

Serota, K. B., Levine, T. R., \& Boster, F. J. (2010). The prevalence of lying in America: Three studies of self-reported lies. Human Communication Research, 36(1), 2-25. doi:10.1111/j.1468-2958.2009.01366.x

Simons, D. J. (2000). Attentional capture and inattentional blindness. Trends in Cognitive Science, 4(4), 147-155.

Sommers, S.R. \& Douglass, A.B. (2007). Context matters: Alibi strength varies according to evaluator perspective. Legal and Criminological Psychology, 12, 41-64.

Stewart-Williams, S. (2007). Altruism among kin vs. nonkin: Effect of costs of help and reciprocal exchange. Evolution and Human Behavior, 28, 193-198.

Strange, D., Dysart, J., \& Loftus, E. F. (2014). Why alibi errors are not necessarily evidence of guilt. Zeitschrift für Psychologie, 222(2), 82-89. doi: 10.1027/2151-2604/a000169

Trivers, R.L. (1971). The evolution of reciprocal altruism. Quarterly Review of Biology. 46, 3557.

Vrij, A. (2008). Detecting lies and deceit: Pitfalls and Opportunities. Second edition. Chichester: John Wiley and Sons. 
Watson, D., Clark, L. A., \& Tellegen, A. (1988). Development and validation of brief measures of positive and negative affect: The PANAS scales. Journal of Personality and Social Psychology, 54(6), 1063-1070. doi:10.1037/0022-3514.54.6.1063

Wells, G. L., Small, M., Penrod, S., Malpass, R. S., Fulero, S. M., \& Brimacombe, C. A. E. (1998). Eyewitness identification procedures: Recommendations for lineups and photospreads. Law and Human Behavior, 22, 603-647. 